\title{
Impact of Particle Injection on Gas Flow at Elevated Pressure: A Numerical Study
}

\author{
Ansan Pokharel \\ ap0118@mix.wvu.edu
}

Follow this and additional works at: https://researchrepository.wvu.edu/etd

Part of the Mechanical Engineering Commons

\section{Recommended Citation}

Pokharel, Ansan, "Impact of Particle Injection on Gas Flow at Elevated Pressure: A Numerical Study" (2020). Graduate Theses, Dissertations, and Problem Reports. 7671.

https://researchrepository.wvu.edu/etd/7671

This Thesis is protected by copyright and/or related rights. It has been brought to you by the The Research Repository @ WVU with permission from the rights-holder(s). You are free to use this Thesis in any way that is permitted by the copyright and related rights legislation that applies to your use. For other uses you must obtain permission from the rights-holder(s) directly, unless additional rights are indicated by a Creative Commons license in the record and/ or on the work itself. This Thesis has been accepted for inclusion in WVU Graduate Theses, Dissertations, and Problem Reports collection by an authorized administrator of The Research Repository @ WVU. For more information, please contact researchrepository@mail.wvu.edu. 
Graduate Theses, Dissertations, and Problem Reports

2020

Impact of Particle Injection on Gas Flow at Elevated Pressure: A Numerical Study

Ansan Pokharel

Follow this and additional works at: https://researchrepository.wvu.edu/etd

Part of the Mechanical Engineering Commons 
Impact of Particle Injection on Gaseous Flow at an Elevated Pressure: A Numerical Study

\author{
Ansan Pokharel \\ Thesis submitted \\ to the Benjamin M. Statler College of \\ Engineering and Mineral Resources \\ at West Virginia University \\ in partial fulfillment of the requirements for the degree of \\ Master of Science in \\ Mechanical Engineering \\ V'yacheslav Akkerman, Ph.D., Chair \\ Ismail Celik, Ph.D. \\ Terence Musho, Ph.D.
}

Department of Mechanical and Aerospace Engineering

West Virginia University

Morgantown, West Virginia

2020

Keywords:

Staged pressurized oxy-fuel combustion (SPOC), phase coupling, particle loading, turbulence dispersion

Reynolds-Averaged Navier-Stokes (RANS) modeling

Copyright 2020 @ Ansan Pokharel 


\section{Abstract \\ Impact of Particle Injection on Gaseous Flow at an Elevated Pressure: A Numerical Study Ansan Pokharel}

Modeling of a turbulent two-phase gaseous-solid flow still faces challenges. The present study is devoted to two-phase flow in an annular pipe (hollow cylinder) operating at an elevated pressure of 15 bar and moderate Reynolds numbers of circa $6 \times 10^{3}$. The influence of the various factors such as the particle loading, the interaction between the phases, and turbulent dispersion - on the flow dynamics is systematically studied by means of the computational simulation employing the ANSYS FLUENT commercial package. To be specific, particle loading with a volumetric fraction of $1.2 \%$ is defined as high particle loading, while the flow with a volumetric fraction of $0.13 \%$ is referred to as low particle loading. In particular, seven various cases for a gas-solid phase flow are investigated:

1) Pure gas flow;

2) Low particle loading two-phase flow with one-way coupling and with turbulence dispersion;

3) Low particle loading two-phase flow with two-way coupling but without turbulence dispersion;

4) Low particle loading two-phase flow with two-way coupling and with turbulence dispersion;

5) High particle loading two-phase flow with one-way coupling and with turbulence dispersion;

6) High particle loading two-phase flow with two-way coupling but without turbulence dispersion;

7) High particle loading two-phase flow with two-way coupling and with turbulence dispersion.

The boundary layer was found to be growing without fluctuations of the turbulent kinetic energy (TKE) for Cases 1, 2, and 5 above. For Case 4, the TKE fluctuations have been identified though appeared not as substantial as in Cases 6 and 7. The author attributes such a difference in the fluctuations to the particle loading. In addition, the onset and development of the flow instability have been observed at a random axial distance in Cases 4, 6, and 7. Such instability is presumably attributed to the two-way coupling with turbulence dispersion in a flow. It is concluded that the particle loading, one-way, or two-way coupling between the phases, and the turbulence dispersion models significantly influence the flow dynamics. The present computational results inspire to perform experimental verification and validation of the simulations, so the simulation results can subsequently be used for the design analysis. 


\section{Acknowledgment}

First, I would like to thank my committee members: Dr. V'yacheslav Akkerman for bestowing a belief on me and providing me with an opportunity to work on this project. I appreciate his immense support and suggestions. In addition, I thank Dr. Ismail Celik for all his invaluable suggestions which propelled me to indulge and focus on the study. I had a wonderful time working with him. Also, I thank Dr. Terence Musho for his entire help. He has always kept his door open for me and I am thankful for that. I feel honored that they were part of my thesis.

I would like to extend my regards to my colleagues, friends, and lab mates for tolerating me in the lab. I am indebted to their assistance and feedback which they provided me while writing the thesis. I would like to extend my sincere gratitude towards the experimental team at Washington University at St. Louis, especially Dr. R. L. Axelbaum for the collaboration and feedback. They are an indispensable part of my work. 
Dedicated to

My Family 


\section{Table of content}

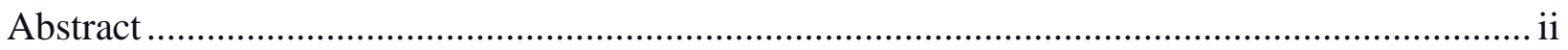

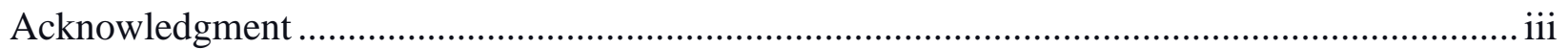

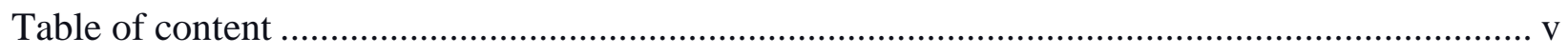

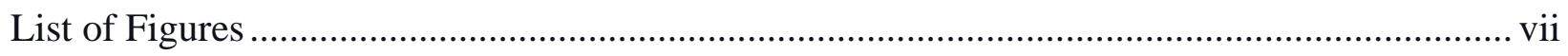

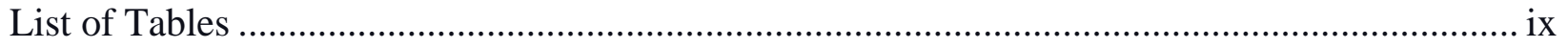

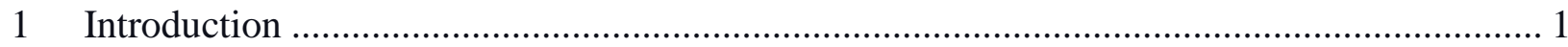

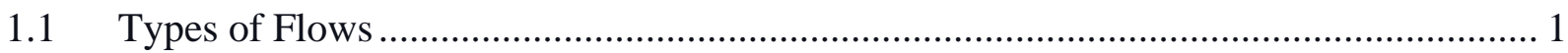

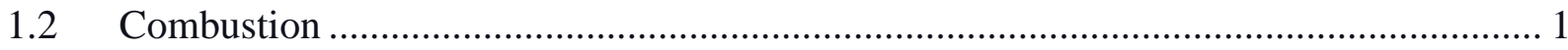

1.2.1 Carbon Capture and Staged Pressurized Oxy-fuel Combustion ............................ 2

2 Motivation and Objectives...................................................................................... 5

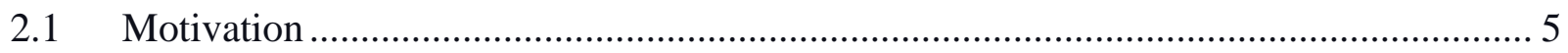

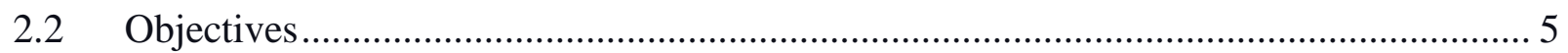

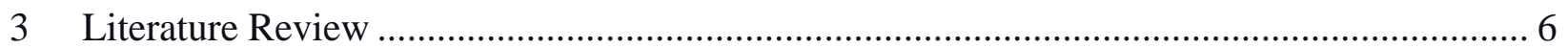

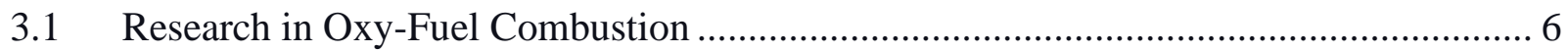

3.2 Previous Studies and Research Gap .......................................................... 7

3.3 Computational Fluid Dynamics and Turbulence Modeling .................................... 9

3.3.1 Computational Fluid Dynamics (CFD) ......................................................... 9 


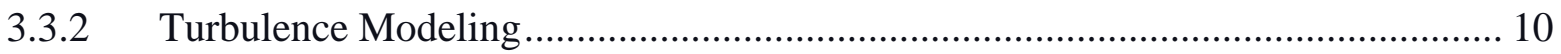

3.3.3 Numerical Modelling of a Gas-Solid Flow ....................................................... 16

3.4 Coupling Between Phases ........................................................................................ 19

3.5 Turbulence Dispersion .................................................................................... 19

4 Methodology

4.1 Geometry and Domain Discretization.......................................................................... 21

4.2 Numerical Method and Boundary Conditions …………….................................... 25

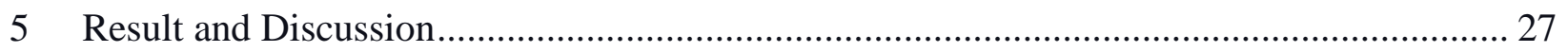

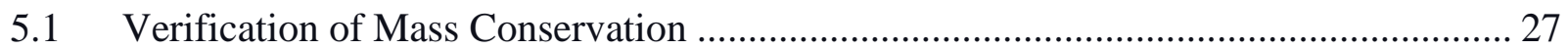

5.2 One-way and Two-way Coupling with and without Turbulence Dispersion.................. 30

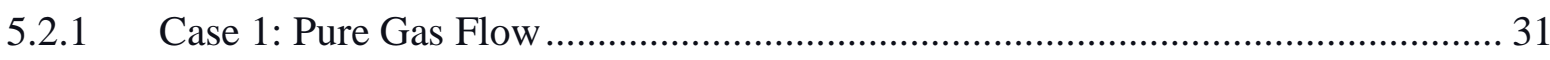

5.2.2 Influence of Gravity in a Flow ……………………........................................... 34

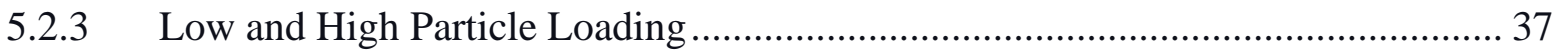

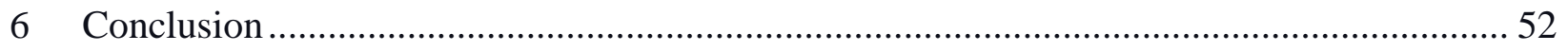

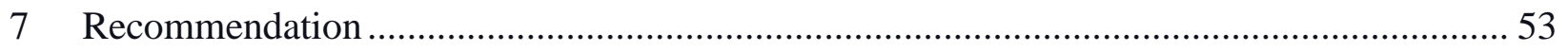

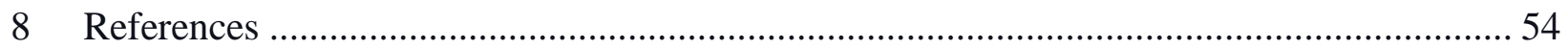




\section{List of Figures}

Figure 1.1: Schematic diagram of an oxy-fuel combustion process [9]................................ 3

Figure 3.1: Schematic diagram of the SPOC system [23]. ................................................ 8

Figure 3.2: Geometry of the reactor and inlet section of SPOC [21]. ................................. 8

Figure 4.1: A wireframe structure of the configuration used............................................... 22

Figure 4.2: The solid structure of the configuration used................................................ 23

Figure 4.3: The section of the geometry with inlet, outlet, and inner wall............................ 23

Figure 4.4: The solid structure of the geometry with inlet, outlet, and inner wall. ................... 24

Figure 4.5: The Wireframe and solid structure of inlet surface (particles injection surface)...... 24

Figure 5.1: A contour of the DPM concentration in the SPOC process [23] ........................... 28

Figure 5.2: A contour of the DPM concentration. .................................................................. 28

Figure 5.3: The particle mass flow rate at the inlet versus time........................................... 29

Figure 5.4: The particle mass flow rate at the outlet versus time. ......................................... 29

Figure 5.5: Difference in particle number entering and exiting from the control volume versus

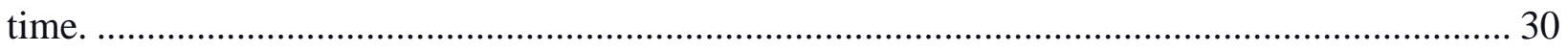

Figure 5.6: Axial velocity contour for a pure gas flow. The vertical black line shows the monitoring

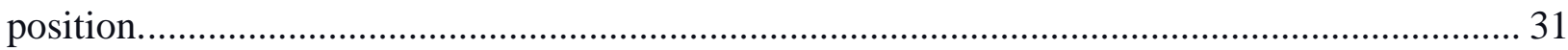

Figure 5.7: Axial velocity of the gas versus radial distance at various axial locations. .............. 32 
Figure 5.8: Axial velocity along with the boundary layer for a pure gas-phase flow 33

Figure 5.9: The TKE along with the boundary layer for a pure gas-phase flow. 34

Figure 5.10: The axial velocity of the gas and the particles at the inlet in the presence of gravity.

Figure 5.11: The axial velocity of the gas and particles at the outlet in the presence of gravity.. 35

Figure 5.12: The axial velocity of the gas and particles at the inlet in the absence of gravity.... 36

Figure 5.13: The axial velocity of the gas and particles at the outlet in the absence of gravity... 36

Figure 5.14: The TKE along the boundary layer for Case 2 ............................................... 37

Figure 5.15: Axial velocity of the gas versus radial distance at various axial locations. ............ 38

Figure 5.16: Axial velocity of the gas versus radial distance at various axial locations............ 39

Figure 5.17: The TKE profile along with the boundary layer for Case 3............................. 40

Figure 5.18: The axial gas velocity along with the boundary layer for Case 3...................... 40

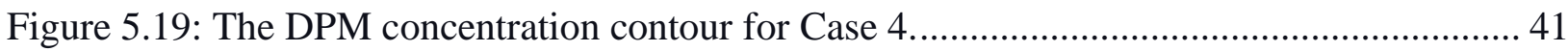

Figure 5.20: Axial velocity of the gas versus radial distance at various axial locations. ............ 42

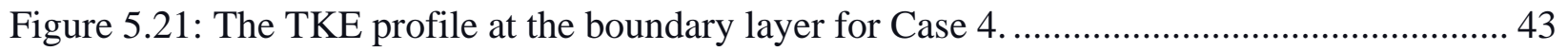

Figure 5.22: The axial velocity of the gas at the boundary layer for Case 4.......................... 43

Figure 5.23: The TKE profile along with the boundary layer for Case 5 ............................... 44

Figure 5.24: Axial velocity of the gas versus radial distance at various axial locations. ........... 45

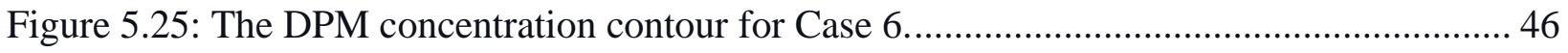

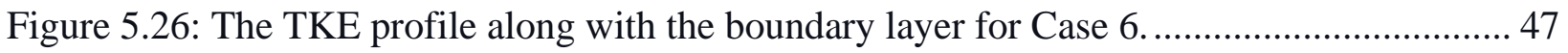

Figure 5.27: The axial velocity of gas along with the boundary layer for Case 6................... 47

Figure 5.28: Axial velocity of the gas versus radial distance at various axial locations. ............ 48

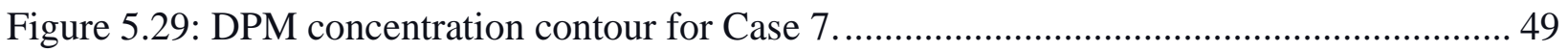

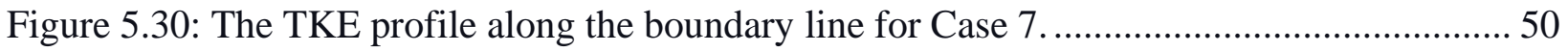


Figure 5.31: Velocity profile of the gas phase along the boundary line for Case $7 \ldots \ldots \ldots \ldots \ldots \ldots . . . .50$

Figure 5.32: Axial velocity of the gas versus radial distance at various axial locations. ............ 51

\section{List of Tables}

Table 1: Domain extents for the geometry used in the simulation...................................... 22

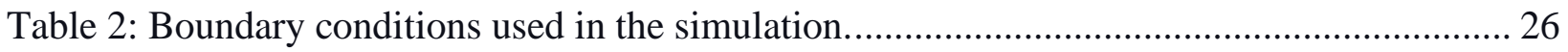

Table 3: Seven different cases developed to study phase coupling, turbulence dispersion, and

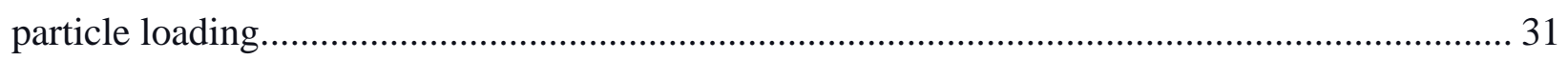

\section{List of Abbreviation}

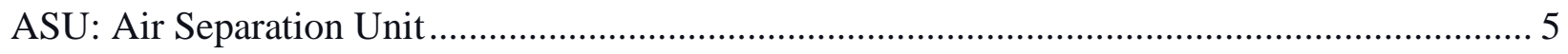

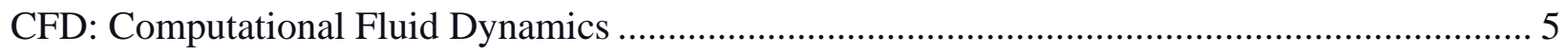

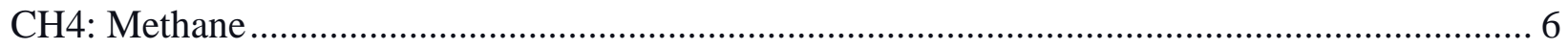

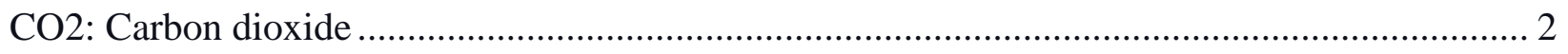

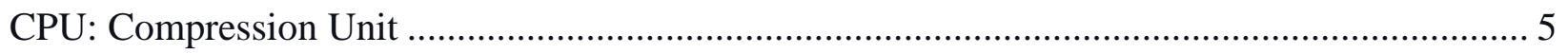

DNS: Direct Numerical Simulation ........................................................................... 10

DPM: Discrete Phase Model......................................................................................... 20

DRW: Discrete Random Walk Model .............................................................................. 23

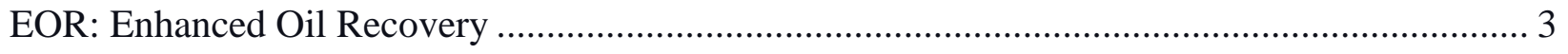

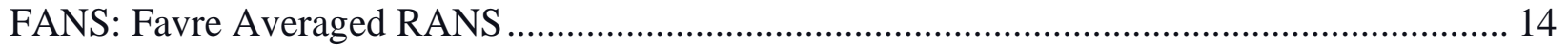

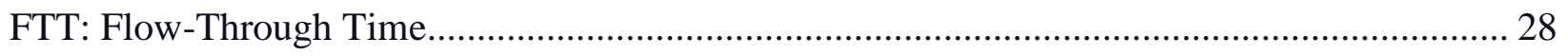

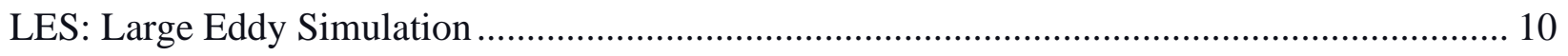

MUSCL: Monotonic Upstream-centered Scheme for Conservation Laws ............................. 29

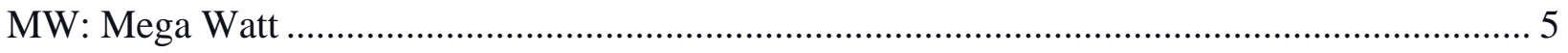




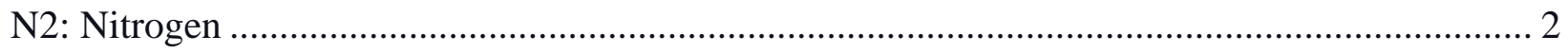

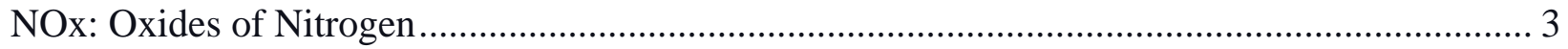

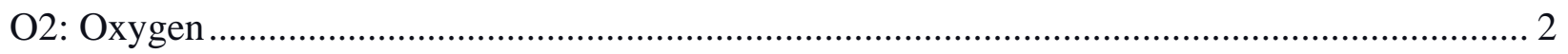

p: Pressure

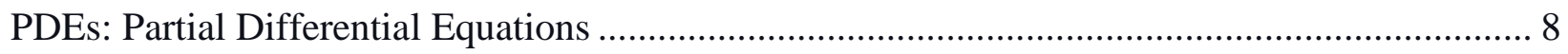

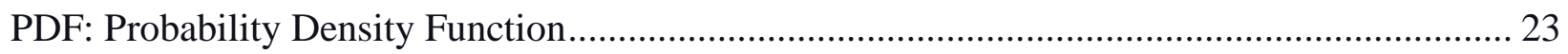

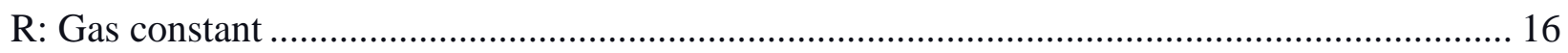

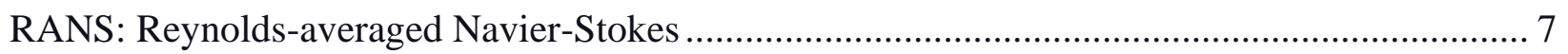

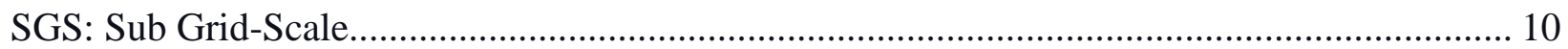

SIMPLE: Semi-Implicit Method for Pressure Linked Equations ................................................... 29

SPOC: Staged Pressurized Oxy-fuel Combustion …………................................................... 2

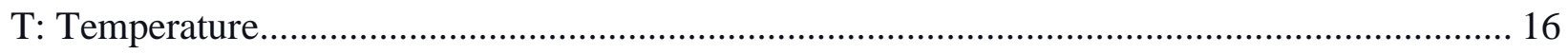

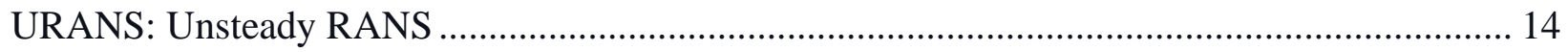

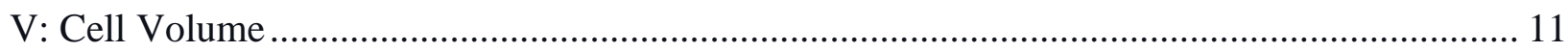

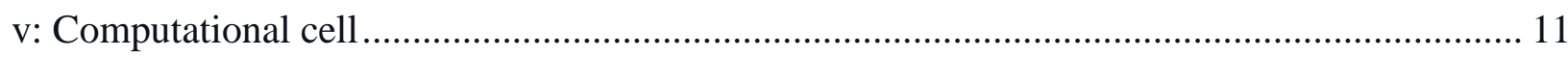

\section{List of symbols}

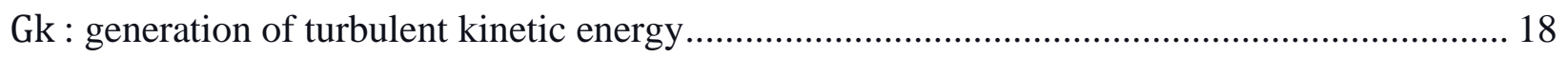

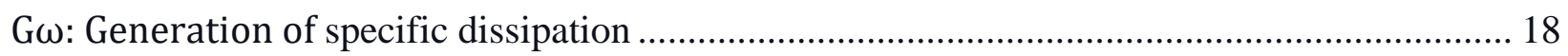

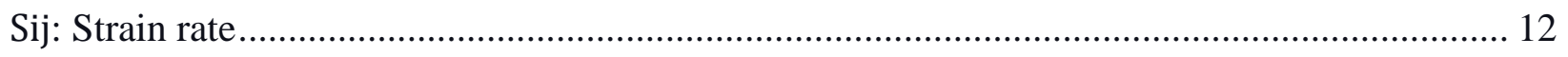

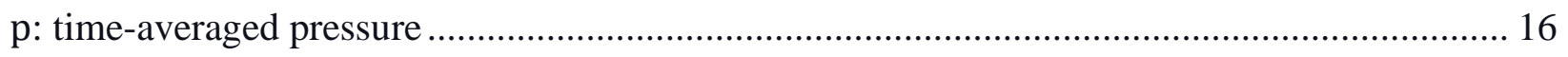

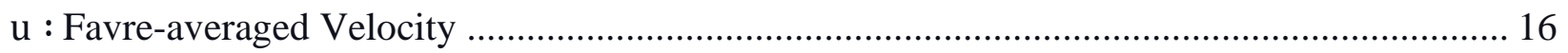

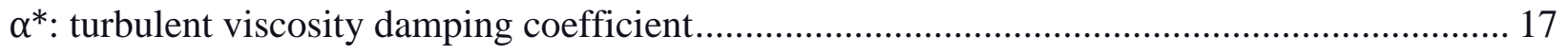

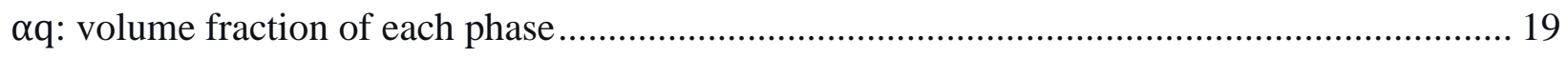




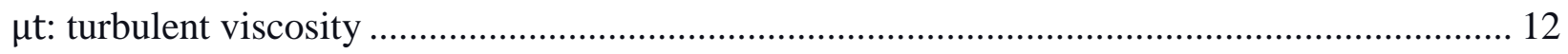

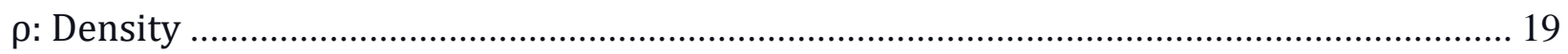

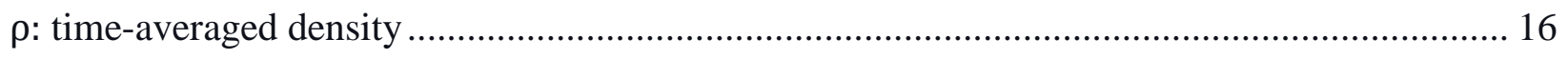

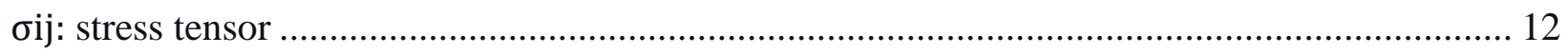

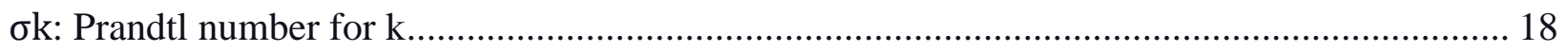

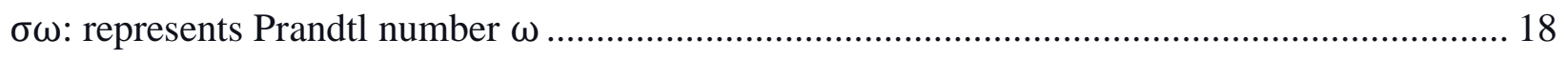

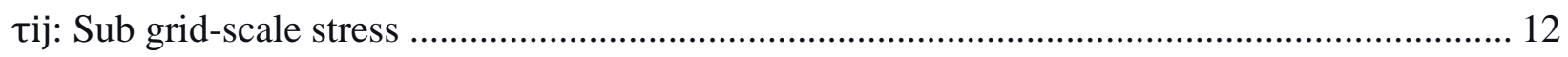

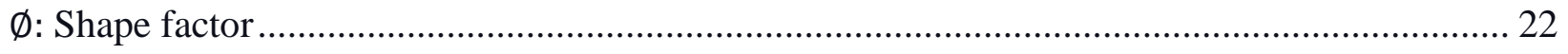

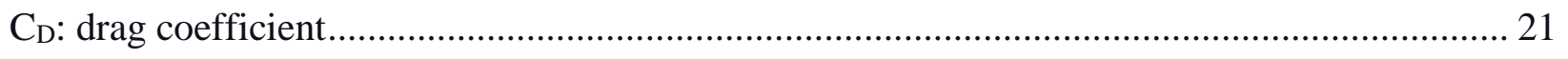

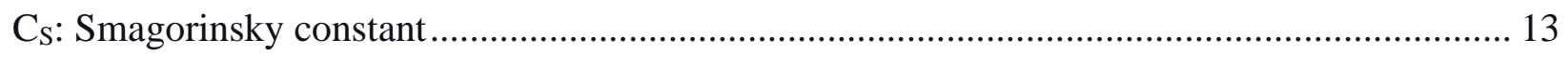

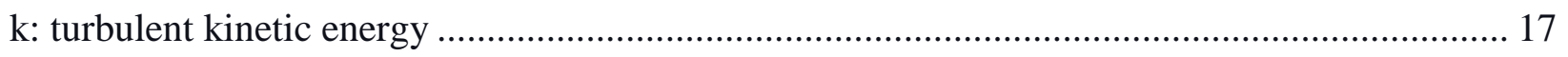

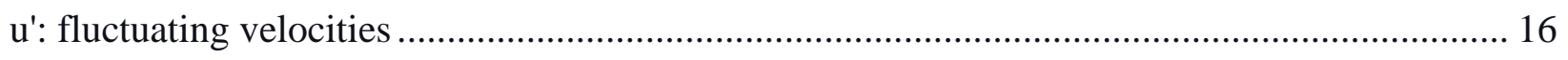

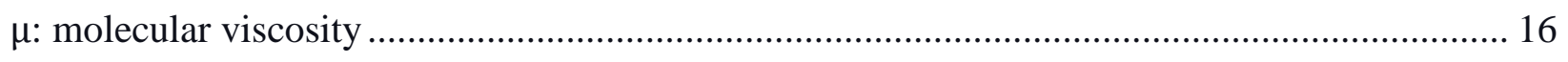

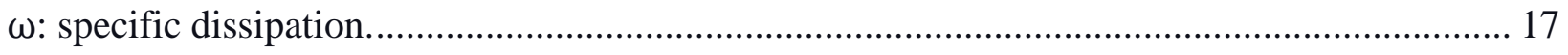




\section{Introduction}

\subsection{Types of Flows}

A multiphase flow can be defined as a fluid flow consisting of more than one phase while a fluid flow with just two distinct components or phases is categorized as two-phase flows. Multiphase, especially, two-phase flows are ubiquitous in the real-world and engineering applications, such as in coal combustion, transportation of oil and gas through a pipe, sewage pipeline, nuclear reactor, pneumatic conveying, and many more. Medical and biological flow processes, critically important blood flow, are also among the examples of multiphase flows. Natural disasters such as avalanches, floods, and landslide are also considered as multiphase flows [1]. The multiphase flows are divided into two distinguishing categories: (I) Dispersed multiphase flow, where the primary (main) phase transports or carries secondary phase, e.g. coal particles carried by air in a burner and (II) the separated multiphase flow that consists of two separate phases unmixed during the flow but being in contact at the shear layer [2-3].

Two-phase flows are classified into three distinct groups: I) Gas-solid flows, where the solid phase is carried by the gas phase, e.g. suspension of the solid particle in the air; II) Gas-liquid flows, which are formed by the combination of the liquid phase and gas phase, which consist of either the same or different volume fraction of phases, e.g. spraying of fuel in engine cylinder along with air; and III) Liquid-solid flows, which are formed by the dispersion of the solid particles in a liquid, e.g. flow in sewage pipe [4].

Multiphase flows occur from a nuclear reactor to a sewage pipe flow. Despite being commonplace, a multiphase flow also has a lot of challenges. Hence, the comprehensive fundamental studies of the multiphase flow processes are utterly important, which can eventually help to enhance the effectiveness of the process and the overall design of better systems.

\subsection{Combustion}

Combustion is defined as an exothermal process, where heat, light, and burnt matter are generated in a chemical reaction between an oxidizer and a fuel. Combustion is categorized as premixed and 
non-premixed (diffusion) [5]. A combustion process, where the mixing of the fuel and oxidizer happens before ignition is regarded as premixed combustion. The common example of premixed combustion is that in spark-ignition engines. Non-premixed or diffusion combustion is defined as a combustion process where the oxidizer and fuel are separated before ignition. A burning candle is a good example of non-premixed combustion. Further, premixed combustion is categorized into the deflagration and detonation regimes. Deflagration, also known as a flame, occurs due to thermal conduction and it spreads at a subsonic speed. In contrast, a combustion front that travels at supersonic speed, because of shock waves is known as a detonation.

Coal, natural gas, petroleum, etc. are the major fossil fuels that are used in transportation, heating, and industrial combustion for energy production. In addition to heat, carbon dioxide $\left(\mathrm{CO}_{2}\right)$ is the primary outcome of combustion. Numerous studies have pointed out $\mathrm{CO}_{2}$ to be the major culprit contribution to global warming [6]. Coal-based power plants are one of the leading sources for $\mathrm{CO}_{2}$ emission. In 2018, 85\% of the world energy demand was supplied from the fossil fuels, of which $27 \%$ was generated from coal [7]. In the same year, the consumption of coal has increased by $1.4 \%$ and its production has grown by $162 \mathrm{Mt}$ (mega tonnes) of oil equivalent. One tonne of oil equivalent is the energy released during the burning of one tonne of crude oil. The growth rate of carbon emission was found to be $2 \%$; which was the highest in seven years [8]. The presence of copious resources and ease in the extraction process has made coal one of the cheapest fossil fuel and its consumption is likely to be increasing until the foreseeable future. Hence, to generate clean energy from coal combustion with the least carbon emission, the design of an efficient coal combustor is required. Staged Pressurized Oxy-Coal Combustion (SPOC) is one such technology.

\subsubsection{Carbon Capture and Staged Pressurized Oxy-fuel Combustion}

$\mathrm{CO}_{2}$ is one of the primary products generated during combustion of a fossil fuel, and it is almost inevitable to generate the heat without any $\mathrm{CO}_{2}$ formation. Consequently, carbon capturing is one of the state-of-the-art technologies developed to mitigate the problem of carbon emissions. The separation of $\mathrm{CO}_{2}$ from other elements/components in a mixture is considered as carbon capturing [9]. Usually, air - consisting of $21 \%$ of oxygen $\left(\mathrm{O}_{2}\right)$ and $78 \%$ of nitrogen $\left(\mathrm{N}_{2}\right)-$ is used as an 
oxidizer during combustion. In addition to heat and $\mathrm{CO}_{2}$, a significant fraction of $\mathrm{N}_{2}$ is generated during air-fuel combustion. Carbon capturing from such a mixture first requires $\mathrm{CO}_{2}$ separation from the product which is energy-consuming.

Oxy-fuel combustion is burning of fuel with almost pure oxygen. Oxygen supersedes air in this process since it significantly decreases the amount of $\mathrm{N}_{2}$ in the products [10]. In air-fuel combustion, $\mathrm{N}_{2}$ acts as an inert gas and absorbs some portion of heat generated during combustion. However, the absence of $\mathrm{N}_{2}$ in the oxy-fuel process increases the adiabatic flame temperature. To mitigate this demand, $\mathrm{CO}_{2}$ is recycled back and injected into the combustor to control/regulate the desired flame temperature. A flue gas produced in this process contains a high $\mathrm{CO}_{2}$ concentration.

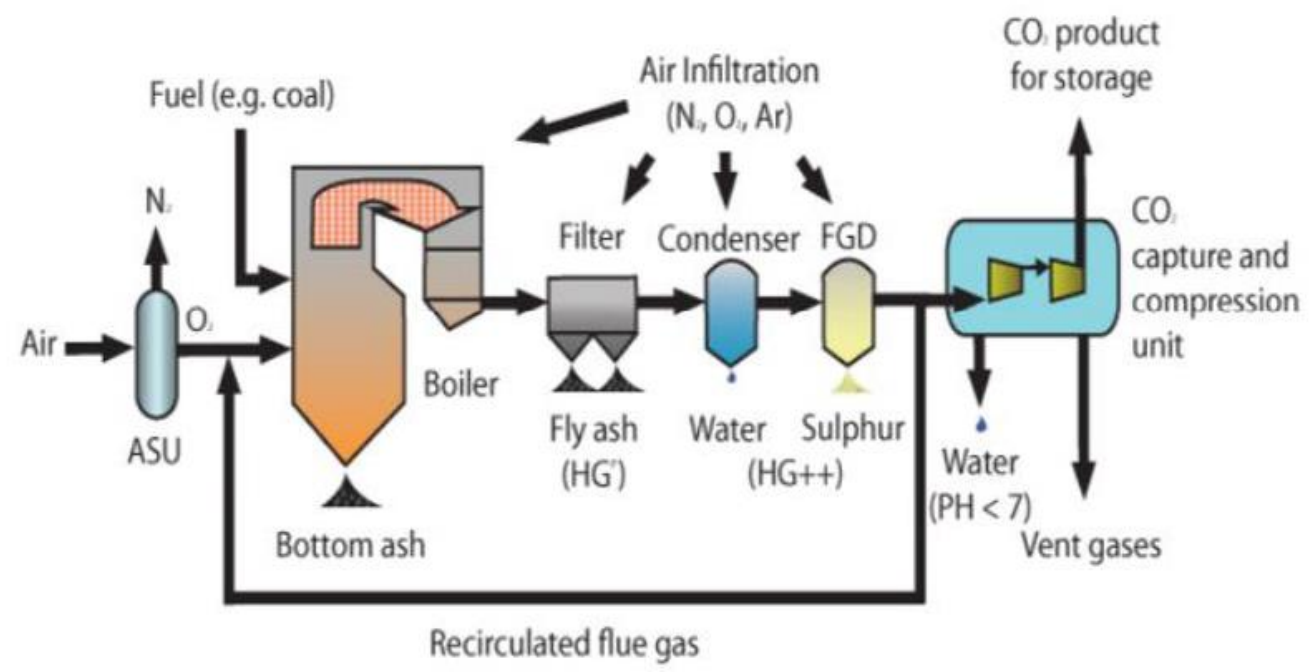

Figure 1.1: Schematic diagram of an oxy-fuel combustion process [9].

Although a high concentration of $\mathrm{CO}_{2}$ is generated during oxy-fuel combustion, the generated $\mathrm{CO}_{2}$ is eventually required to be pressurized either for sequestration or enhanced oil recovery (EOR). Staged pressurized oxy-fuel combustion, hence referred to as SPOC, is a process occurring at elevated pressures, where the injection of fuel is staged to control the high flame temperature. Oxyfuel combustion at an elevated pressure excludes the necessity to pressurize the $\mathrm{CO}_{2}$ generated as a product of the combustion process and increases the dew point of the flue gas. The latent heat of flue gas moisture generated at the elevated pressure can be used back in the operation cycle, which 
eventually increases the overall efficiency of the operating system [12]. Besides, the operation of the SPOC system at high pressure decreases the volume of the gas which eventually decreases the size of the equipment used in the system and hence the total cost associated with the system. Also, there is no formation of nitrogen oxides $\left(\mathrm{NO}_{\mathrm{x}}\right)$, which decreases the cost of separation and purification of $\mathrm{CO}_{2}$ from $\mathrm{NO}_{\mathrm{x}}$. This makes the $\mathrm{SPOC}$ process one of the most promising carboncapturing technology. 


\section{Motivation and Objectives}

\subsection{Motivation}

An increase in coal production and consumption, which is not likely to decrease in the forthcoming future, has necessitated the need of an effective coal combustor that can generate clean energy via burning of coal. The SPOC and flameless combustion technology (ISOTHERM) are two major oxy-fuel combustion technologies operating at elevated pressures. The requirement of recycling a lesser volume of flue gas in the SPOC as compared to the ISOTHERM decreases its energy penalty [13]; consequently, the SPOC is expected to run with higher efficiency.

Arguably, two-phase flows are commonplace in real-world applications and have a wide scope. The SPOC primarily employs coal particles as the fuel, which makes the injection process inside the SPOC a two-phase (gas-solid flow) system. The SPOC, which is at the first stage of the study, is one of the cutting-edge technologies and has shown promising results; however, the fundamental understanding of flow inside SPOC is still required. The present thesis is primarily focused on the fundamental study of the gas-solid (coal particle) behavior inside a pressurized system in a vertical annular tube. Specifically, a thorough research is conducted to understand the flow dynamics at the inlet section of the SPOC geometry. However, this research should not be limited to the SPOC and can further be used to understand the fluid-particle dynamics in general.

\subsection{Objectives}

The dynamics of gas-particle (air-coal particles) flow in the inlet section of the SPOC burner has being investigated. The main objectives of this study were:

1. To design and develop the geometry and mesh for the inlet section of the SPOC burner using the ICEM (commercial software primarily used for mesh generation).

2. To develop an accurate gas-solid turbulence model using the Ansys FLUENT package.

3. To investigate the role of particle loading in the flow field.

4. To study the role of turbulence dispersion in the flow field.

5. To study the difference between one-way and two-way coupling.

The details of the turbulence dispersion and phase coupling are provided in the following section. 


\section{Literature Review}

Giacinto et. al. [14] conducted an investigation concluding that particle loading (particle volume fraction) is one of the primary parameters determining the choice between one-way and two-way coupling for a computational simulation of a two-phase flow. In the parametric study of a gasparticle flow in a vertical duct conducted by Liu et al. [15], it was found that the segregation of the particle transited towards the wall from the center, with the increase in the solid phase loading, considering the particles to be elastic. In contrast, considered as inelastic, the particles were found to be segregated towards the center of the channel. Sinclair et al. [16] investigated a gas-particle flow in a vertical pipe considering particle-particle interaction. Segregation of both the phases was found in the radial direction. Likewise, a similar numerical study was conducted by Kartushinsky et al. [17], for a 3D gas-solid flow but in a horizontal pipe. An asymmetric flow field was observed, with the gravity being attributed to be the cause of such a flow phenomenon. In the semi-empirical model for an upward vertical two-phase flow system, developed by Rhodes et al. [18], it was implied that the radial solids concentration profile in flow regime is governed by the cross-section area of a pipe. Ding et al. [19] observed the laminar-to-turbulent transition at the Reynolds number of $R e=620$, which is substantially less than the conventional values for such a transition.

\subsection{Research in Oxy-Fuel Combustion}

Despite being at the primary stage of development, a significant research has been conducted on the oxy-fuel combustion technologies, including the SPOC, which has led to the efficient operation of power plants with the various capacity. For the past decade, the majority of the research efforts on oxy-fuel technologies is being focused on the development of the combustor/boiler [10].

Using Aspen Plus, Gopan et al. [12] studied the effect of operating pressure and fuel moisture on net plant efficiency and attributed the SPOC process to increase the net plant efficiency by $6 \%$ as compared to the first-generation, oxy-combustion process. No significant rise in a plant efficiency was observed for the operating condition beyond 16 bar. Xia et al. [20] ran a computational fluid dynamic (CFD) simulation at an elevated pressure of 16 bar with low flue gas recycle for a 1540 
MW SPOC system. For efficient combustion, conical geometry of the combustor was implemented that generated a relatively longer and thinner flame with uniform distribution of wall heat flux as compared to conventional design. With an increase in the operating pressure of the combustor, the energy requirement for the air separating unit (ASU) and compression unit (CPU) increases. Hong et al. [21] conducted a holistic pressure-sensitive analysis to calculate the optimal pressure required to run an efficient oxy-fuel combustion power cycle. It was found that, with an increase in the operating pressure, the ASU compression consumed more energy, while the CPU required less energy. Hence, the maximum efficiency was found to be gained at around 10 bar of operating pressure. Lasek et al. [22] studied, experimentally, the emissions of $\mathrm{NO}_{\mathrm{x}}, \mathrm{N}_{2} \mathrm{O}$, and other elements in an oxy-fuel combustor at elevated pressures. The emissions of $\mathrm{SO}_{2}, \mathrm{NO}$, and $\mathrm{N}_{2} \mathrm{O}$ have been found to be significantly curtailed with an increase in the operating pressure.

\subsection{Previous Studies and Research Gap}

Udochukwu [23] performed a numerical investigation on the first stage combustor (out of four) for the SPOC system being developed at the Washington University in St. Louis, MO (WUSTL). A schematic of the overall SPOC process is depicted in Fig. 3.1, while Fig. 3.2 delineates geometry of the combustor and the inlet section of the SPOC. To maintain the adiabatic flame temperature, which is the nominal temperature generated during the combustion with no energy loss [24], the combustion process is divided into four stages [25]. 


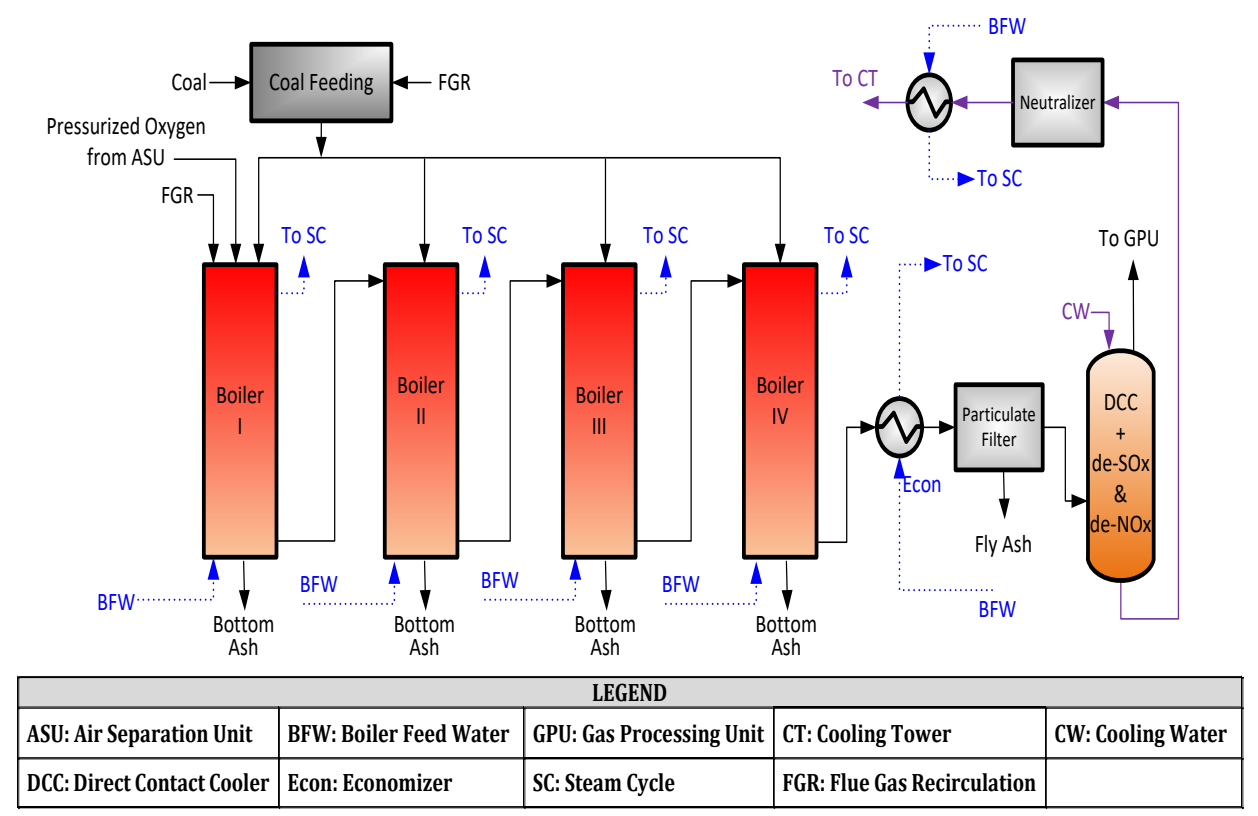

Figure 3.1: Schematic diagram of the SPOC system [23].

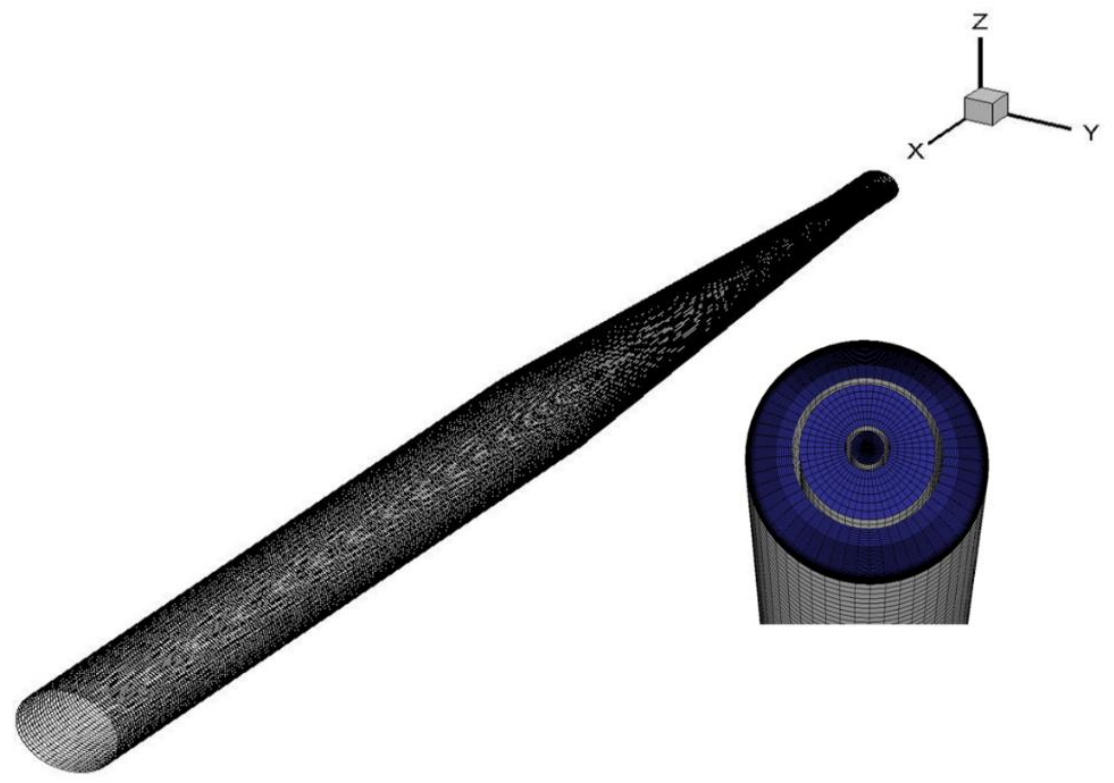

Figure 3.2: Geometry of the reactor and inlet section of SPOC [21].

The geometry in Fig. 3.2. consists of three (primarily, secondary, and tertiary) inlet sections. The injection of a small fraction of recycled $\mathrm{CO}_{2}$ along with excessive $\mathrm{O}_{2}$ prevented the generation of excessive undesired flame temperature. Pressurized (15 bar) $\mathrm{CO}_{2}$ and $\mathrm{O}_{2}$ at various mole fractions 
are injected together from the primary inlet and tertiary inlet however, pulverized coal particles along with recirculated $\mathrm{CO}_{2}$ and methane $\left(\mathrm{CH}_{4}\right)$ are injected through the secondary inlet. A small amount of $\mathrm{CH}_{4}$ is injected to assist the flame ignition and sustain the combustion.

During this investigation, Udochukwu [23] ran steady and unsteady Reynolds-averaged NavierStokes (RANS) simulation, described in the later sections. Despite the use of perfectly symmetric geometry, the non-symmetric and oscillating flame inside the combustor was observed. Injection of the coal particles (solid) in the gas was found to be one of the causes of this problem. An increase in the volumetric flow rate of coal carrier (i.e. $\mathrm{CO}_{2}$ ) was found to mitigate this problem, to some extent. While this research has unveiled some magnificent results, it lacked the explanation of how particle disturbs the gas flow field. In particular, it has not been vividly explained if the inception of the oscillations happens prior to flame ignition or after it. An increase in the volumetric flow rate was attributed to the decreasing oscillations, but it has not been clarified why/how an increase in the volumetric flow rate attenuates the problem of the flame oscillations. This required a separate thorough investigation of the particle-gas interaction at the secondary inlet, which constitutes the content of the present work. Specifically, this thesis is primarily focused on understanding the particle-gas interaction in the second inlet and unveiling the influence of the volume fraction of the particle (or mass flow rate) in the flow field.

\subsection{Computational Fluid Dynamics and Turbulence Modeling}

\subsubsection{Computational Fluid Dynamics (CFD)}

The CFD is a numerical approach used for solving the three-dimensional (sometimes lower) and transient partial differential equations (PDEs) [26]. Along with the combination of mass and heat transfer, the CFD allows a comprehensive analysis of the flow field with the assistance of powerful computers [27]. The Navier-Stokes (N-S) equations, which are derived to mathematically delineate various flows including turbulent flows, are three-dimensional (3D), transient (time-dependent) and non-linear PDEs. The presence of non-linearity in the equation creates the closure problem and foils the generation of the exact solution. Nevertheless, various computational tools have been 
devised that predict the turbulent phenomena based on the continuity, momentum, and energy equations. Initial and boundary conditions along with various physical properties as well as proper spatial discretization and time formulation must be supplied to make an accurate CFD prediction.

Even though computational facilities has largely increased in past decades, the results generated from the CFD tools are never exact which can be accredited to various sources of errors [28]. However, CFD tools should not be understood otherwise as it has lots of advantages. To mention a few:

I. CFD simulations generate results with lesser cost as compared to the experiments. Interpretation of the results might require some time and human power with the working experience of CFD tools [28].

II. The study of turbulence requires a frequent change in parameters (e.g. pressure, temperature, velocity). Often, the dimensions of geometry are changed and hence the experimental process must be reinitialized to generate the results. Therefore, such repetitive change of dimension and parameters are daunting and expensive. The CFD appears very useful in such situations and helps in design optimization.

III. The study of the flow field in detail may not be possible in an experimental setup, in particular, data at some point in a domain cannot be extracted because of extreme flow conditions. Then the CFD simulations can easily predict and extract data that otherwise could not have been possible to generate from the experiment [29].

IV. The CFD can help to predict the mishap or explosion (that can occur during the experiment) and save life and property.

\subsubsection{Turbulence Modeling}

Turbulence is one of the most mysterious and complicated phenomena existing in the nature and industrial flows. One of the basic traits of turbulence flow is the presence of random and chaotic fluctuations, with a wide spectrum of length and time scales involved in the problem. Generating the analytical solution that can fully explain such a random behavior has always been an elusive 
quest. The statistical approach is, however, found to produce a solution that helps to understand certain turbulence properties. Motion or a movement of molecules in a turbulent flow field is in the irregular chaotic path which makes a turbulent flow different from a laminar one. Also, enhanced diffusion that prompts mass, momentum, and energy transfer makes turbulence a useful phenomenon in engineering applications [30]. Mathieu et al. [31] have described turbulence to have various physical properties including small scale random vorticity. Random mixing - due to eddies of various sizes and scale - of oxidizer and fuel is the cornerstone for efficient turbulent combustion, which makes it essential to consider turbulence in the combustion process. In the case of gas-solid combustion, it is also necessary to understand the influence of the particles on the gas and vice versa. The role of particle turbulence dispersion in the gaseous flow field should also be thoroughly understood.

A proper grid resolution, numerical algorithm, and turbulence modeling are three factors involved in the CFD simulations. A correct choice of a suitable, problem-oriented turbulence model is very important for capturing the essence of the turbulent flow. Various computational methods are developed and available to solve the turbulent flow problems. These methods are classified into three categories and use different approaches to solve the set of PDEs for a turbulent flow. As detailed below, these three tools are:

I. Direct Numerical Simulations (DNS).

II. Reynolds Averaged Navier-Stokes (RANS) Simulations

III. Large Eddy Simulations (LES).

\subsubsection{Direct Numerical Simulations (DNS)}

Turbulence flow consists of a wide spectrum (in terms of size and frequency) of eddies. Among them, the smallest possible eddies are referred to as the Kolmogorov scale, while the largest eddies determine the integral turbulent length scale. The DNS is a numerical approach to solve the set of PDEs governing a flow without introducing any model. The DNS resolves eddies of all length scales, thereby requiring the mesh of size smaller than the smallest eddies [32]. Since the DNS requires a proper numerical scheme to minimize the dispersion and dissipation errors along with 
fine mesh resolution and smaller time step, the only error produced in this method is from the residual approximation contained in the numerical scheme [33]. The computational cost of the DNS method is estimated to be $\propto \operatorname{Re}^{9 / 4}$; that is why the applications of the DNS method are restricted to flows with a lower Reynolds number [34]. Nevertheless, the advent of highperformance supercomputers is likely to change the scenario.

\subsubsection{Large Eddy Simulation (LES)}

Unlike the DNS, where eddies of all scales are resolved, the LES filters out the small eddies and resolves the larger ones. The space filtering techniques are applied to separate the larger eddies from the smaller ones. However, the effect of the unresolved smaller eddies on the flow field is incorporated by the means of the so-called sub-grid scale (SGS) models [35]. The larger eddies are anisotropic; they interact with the mean flow and extract energy from it. Also, while the larger eddies depend on the domain geometry and boundary conditions, the smaller eddies tend to be isotropic, universal, and independent of geometry, which justifies their filtration from the larger eddies. Because the smaller eddies are modeled, the LES allows the usage of a coarser mesh as compared to the DNS [36]. Hence, the LES computational costs and time are significantly lowered. A suitable filtering function and a cutoff width to separate and resolve large scale eddies are required. All eddies smaller than a cutoff width are modeled. The PDEs governing the flow are then operated with this filtering function, which excludes the information of smaller eddies. Nevertheless, the interaction effect between the resolved and unresolved eddies is captured using the SGS model [35]. A variable obtained after the filtration of $\phi\left(x^{\prime}\right)$ is defined as:

$$
\bar{\phi}(x)=\int_{D} \phi\left(x^{\prime}\right) G\left(x, x^{\prime}\right) d x^{\prime}
$$

Where, $D$ is the computational domain and $G\left(x, x^{\prime}\right)$ is the filtering function. The Ansys FLUENT implicitly generates the filtering function for a finite volume as:

$$
G\left(x, x^{\prime}\right)= \begin{cases}\frac{1}{V}, & x^{\prime} \in v \\ 0, & x^{\prime} \notin v\end{cases}
$$

Where, $V$ is the volume cell and $v$ is the computational cell. Hence 


$$
\bar{\phi}(x)=\frac{1}{V} \int_{v} \phi\left(x^{\prime}\right) d x^{\prime} . \quad x^{\prime} \in v
$$

Filtering the continuity and N-S equation transforms them into

$$
\begin{gathered}
\frac{\partial \rho}{\partial t}+\frac{\partial \rho \bar{u}_{i}}{\partial x_{i}}=0 \\
\frac{\partial\left(\rho \bar{u}_{i}\right)}{\partial t}+\frac{\partial\left(\rho \bar{u}_{i} \bar{u}_{j}\right)}{\partial x_{j}}=\frac{\partial\left(\sigma_{i j}\right)}{\partial x_{j}}-\frac{\partial(\bar{p})}{\partial x \bar{i}}-\frac{\partial \tau_{i j}}{\partial x_{j}},
\end{gathered}
$$

respectively. Here, $\sigma_{i j}$ is the stress tensor and $\tau_{i j}$ is the SGS stress, which represents the influence of the unresolved eddies in the flow field and it is defined as:

$$
\tau_{i j}=\rho \overline{u_{\imath} u_{\jmath}}-\rho \bar{u}_{i} \bar{u}_{\jmath}
$$

The fluctuating terms in the SGS stress are unknown. The most challenging aspect of the LES is an accurate modeling of the SGS stress. The Ansys FLUENT employs the Boussinesq hypothesis to relate the SGS stress with the artificial viscosity termed as SGS the turbulent viscosity $\left(\mu_{t}\right)$ and the strain rate $\left(\bar{S}_{i j}\right)$. Mathematically, it is formulated as:

$$
\begin{gathered}
\tau_{i j}-\frac{1}{3} \tau_{k k} \delta_{i j}=-2 \mu_{t} \bar{S}_{i j}, \\
\bar{S}_{i j} \equiv \frac{1}{2}\left(\frac{\partial \bar{u}_{i}}{\partial x_{j}}+\frac{\partial \bar{u}_{j}}{\partial x_{i}}\right) .
\end{gathered}
$$

Four different options - the Smagorinsky-Lilly model, the dynamic Smagorinsky-Lilly model, the WALE model, and the dynamic kinetic energy SGS model - are available in the ANSYS FLUENT to model the SGS viscosity [36]. Among all other options, the Smagorinsky-Lilly model [37] is mostly used. This is a simple model, which relates the mixing length $L_{s}$ with the eddy viscosity for sub-grid-scale as [37]

$$
\begin{gathered}
\mu_{t}=\rho L_{s}^{2}|\bar{S}| \\
|\bar{S}| \equiv \sqrt{22_{i j} \bar{S}_{i j}}
\end{gathered}
$$


In the Ansys FLUENT, the mixing length $L_{s}$ is computed through the von Karman constant $k, d$ the distance to the closest wall, the Smagorinsky constant $C_{s}$, and the local sub-grid-scale $\Delta$ as

$$
\begin{gathered}
L_{s}=\min \left(k d, C_{s} \Delta\right), \\
\Delta=V^{1 / 3} .
\end{gathered}
$$

\subsubsection{Reynolds-Averaged Navier-Stokes (RANS) Simulation}

In this method, numerical study of a flow is done by performing the time averaging (Reynolds averaging) on the PDEs, continuity, and N-S equation that governs the fluid flow. Reynolds averaging is a mathematical procedure of decomposing the solution variables into the mean and the fluctuating components. The extra term, also called the Reynolds stress term, which depicts the interaction of various turbulent fluctuation is generated as a result of such a decomposition. Proper modeling of the Reynolds stress to tackle the issue of the closure problem is the cornerstone behind the RANS simulation. Therefore, in the RANS simulation, in lieu of resolving the entire scale of eddies or filtering out some of them, all turbulence scales are modeled. Doing so largely saves the computational time and cost [38]: this is the main advantage of the RANS simulation as compared to the DNS and LES. Time-dependent RANS simulations are usually referred to as unsteady RANS (URANS), while the RANS based on the decomposition of various parameters with density weighted time average is defined as Favre-averaged RANS (FANS). The detail mathematical equations are below. The Reynolds decomposition is defined as:

$$
\begin{aligned}
\rho & =\bar{\rho}+\rho^{\prime}, \\
u_{i} & =\bar{u}_{i}+u_{i}^{\prime}, \\
p & =\bar{p}+p^{\prime} .
\end{aligned}
$$

Applying the Reynolds decomposition to the continuity and momentum equations followed by the ensemble averaging yields:

$$
\frac{\partial \bar{\rho}}{\partial t}+\frac{\partial\left(\bar{\rho} \widetilde{u}_{i}\right)}{\partial x_{i}}=0
$$




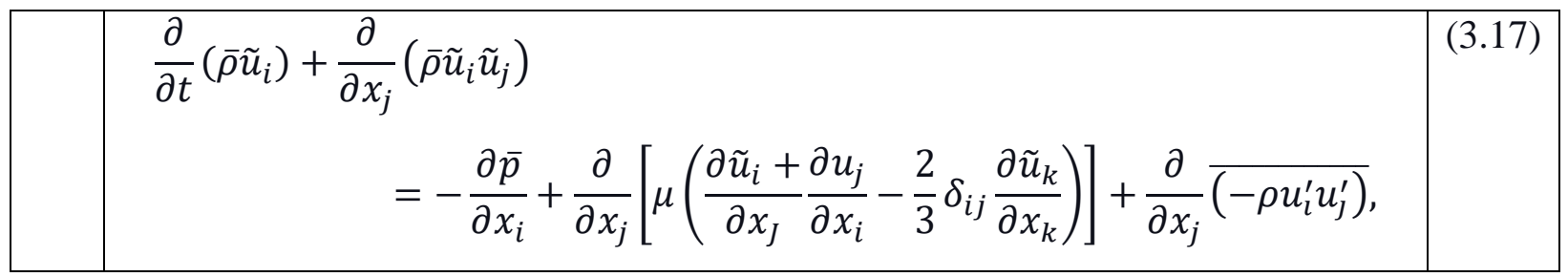

$$
\begin{aligned}
& \delta_{i j}=\left\{\begin{array}{ll}
1 & \mathrm{i}=j \\
0 & \mathrm{i} \neq j
\end{array} .\right.
\end{aligned}
$$

Here $\tilde{u}_{i}, \tilde{u}_{j}$ and $\tilde{u}_{k}$ are three Favre-averaged velocities, $u_{i}^{\prime}$ and $u_{j}^{\prime}$ are the fluctuating velocities, $\mu$ is the molecular viscosity of the fluid, and $\bar{\rho}$ and $\bar{p}$ are time averaged density and pressure respectively [39]. Pressure and velocity in the above equations are coupled by the ideal gas law

$$
p=\rho R T,
$$

where $R$ and $T$ are the specific gas constant and temperature. The term $\overline{\left(-\rho u_{\imath}^{\prime} u_{\jmath}^{\prime}\right)}$ in Eq. (3.17) is the definition of the Reynolds stress. It describes the interaction between the turbulent fluctuations in various directions. The value of the Reynolds stress cannot be derived analytically, hence, it becomes impossible to generate the analytical solution for the $\mathrm{N}-\mathrm{S}$ equations. This closure problem now can numerically be solved by using the Boussinesq hypothesis. Similar to a laminar flow, the dependence of turbulence shear stress and mean rate of the strain are assumed to be linear. This approximation is based on the observation that the mixing caused by large and energetic turbulence eddies dominates the momentum transfer in turbulence flow [40]. Analytically, it is formulated as:

$$
\tau_{i j}=\overline{\left(-\rho u_{\imath}^{\prime} u_{j}^{\prime}\right)} \approx \mu_{t}\left(\frac{\partial \tilde{u}_{i}}{\partial x_{j}}+\frac{\partial \tilde{u}_{j}}{\partial x_{i}}-\frac{2}{3} \delta_{i j} \frac{\partial \tilde{u}_{k}}{\partial x_{k}}\right)-\frac{2}{3} \bar{\rho} k \delta_{i j} .
$$

The proportionality factor $\mu_{t}$ here is the eddy viscosity. It is not a physical property of a flow, such as molecular viscosity, but just a mathematical function of a flow condition and is highly affected by the flow history. The quantity $k$ in Eq. (3.23) is the specific turbulent kinetic energy defines as

$$
k=\frac{1}{2}\left(\overline{{u^{\prime}}^{2}}+\overline{{v^{\prime 2}}^{2}}+\overline{{w^{\prime}}^{2}}\right) \text {. }
$$

Based on additional transport equations, there exist several RANS models. Here, I employed the $k$ - $\omega$ model. The shear stress transport (SST) $k$ - $\omega$ model predicts the flow dynamic much better, both in the free stream and near the wall. For the $k-\omega$ model, the eddy viscosity is defined by: 


$$
\mu_{t}=\frac{\rho k}{\omega} \frac{1}{\max \left[\frac{1}{\alpha^{*}}, \frac{s F_{2}}{\alpha_{1} \omega}\right]},
$$

with

$$
\omega=\epsilon / k \text {. }
$$

Various constants such as $\mathrm{S}, \mathrm{F}_{2}$ and $\alpha^{*}$ are strain rate magnitude, blending function, and turbulent viscosity damping coefficient. The quantity $\omega$ represents the specific dissipation rate which is the ratio of the rate of dissipation of turbulent kinetic energy $(\epsilon)$ and the specific turbulence kinetic energy $(k)$. Now, the specific turbulence kinetic energy (TKE) and specific dissipation rate are obtained by solving the following equations

$$
\begin{gathered}
\frac{\partial(\rho k)}{\partial t}+\frac{\partial\left(\rho k u_{i}\right)}{\partial x_{i}}=\frac{\partial}{\partial x_{j}}\left(\Gamma_{k} \frac{\partial k}{\partial x_{j}}\right)+\tilde{G}_{k}-Y_{k} \\
\frac{\partial(\rho \omega)}{\partial t}+\frac{\partial\left(\rho \omega u_{i}\right)}{\partial x_{i}}+\frac{\partial}{\partial x_{j}}\left(\Gamma_{\omega} \frac{\partial k}{\partial x_{j}}\right)+\tilde{G}_{\omega}-Y_{\omega}+D_{\omega}, \\
\Gamma_{k}=\mu+\frac{\mu_{t}}{\sigma_{k}} \\
\Gamma_{\omega}=\mu+\frac{\mu_{t}}{\sigma_{\omega}}
\end{gathered}
$$

In the above equations, $\tilde{G}_{k}$ and $\tilde{G}_{\omega}$ represents the generation of the specific TKE and the specific dissipation respectively. Likewise, $\Gamma_{k}$ and $\Gamma_{\omega}$ represents the effective diffusivity while $Y_{k}$ and $Y_{\omega}$ denotes the dissipation of the TKE and specific dissipation. The value $D_{\omega}$ represents the cross diffusion while $\sigma_{k}$ and $\sigma_{\omega}$ designate the Prandtl number (Pr) for $k$ and $\omega$, respectively [39].

\subsubsection{Numerical Modelling of a Gas-Solid Flow}

The Ansys FLUENT offers various options for the gas-solid particle modeling which are based on the two principles: (I) the Eulerian-Eulerian (EE) and (II) Eulerian-Lagrangian (EL) approaches.

\subsubsection{Eulerian-Eulerian approach}

The Eulerian-Eulerian (EE), also known as the two-fluid, model treats both the phases as a fully inter-penetrating continuum medium. Different sets of the conservation equations are employed to 
describe the two phases (both fluid), with the interaction terms representing the coupling between the phases [41]. The volumetric fractions defined as the space occupied by each phase are used for representing the individual phases. The volumetric fraction of the particles plays a pivotal role in the modeling of particle-laden flow [42]. The volume of phase $q$ is calculated as

$$
V_{q}=\int_{V} \alpha_{q} \mathrm{~d} V
$$

where $\alpha_{q}$ is a volumetric fraction of each phase and it sums up to 1 for all the phases. The effective density of phase $q$, having a density $\rho_{q}$, is represented by:

$$
\hat{\rho}_{q}=\alpha_{q} \rho_{q}
$$

The continuity equation is used for calculating the volume fraction of phase $q$ with velocity $\vec{v}_{q}$,

$$
\frac{\partial}{\partial t}\left(\alpha_{q} \rho_{q}\right)+\nabla \cdot\left(\alpha_{q} \rho_{q} \vec{v}_{q}\right)=0
$$

Likewise, the momentum equation for phase $q$ reads

$$
\frac{\partial}{\partial t}\left(\alpha_{q} \rho_{q} \vec{v}_{q}\right)+\nabla \cdot\left(\alpha_{q} \rho_{q} \vec{v}_{q} \vec{v}_{q}\right)=-\alpha_{q} \nabla \mathrm{p}+\nabla \cdot \overline{\overline{\tau_{q}}}+\alpha_{q} \rho_{q} \vec{g}+\sum_{p=1}^{n}\left(\vec{R}_{p q}\right)+\vec{F}
$$

where $\overline{\overline{\tau_{q}}}, \vec{g}$ and $\vec{F}$ stand for the stress-strain tensor, acceleration due to gravity, and various forces acting in a flow, respectively, while $\vec{R}_{p q}$ describes the interaction between the phases.

\subsubsection{Eulerian-Lagrangian Approach}

The Eulerian-Lagrangian (EL) approach considers the fluid/gas phase as a continuum medium, while the particles are tracked using the Lagrangian approach by considering it as a discrete phase. Unlike the EE mode, this approach provides more latitude in the flow analysis, with a wide variation range in particle velocity, size, type, shape [43].

The momentum equation governing the gas phase is identical as the Eulerian-Eulerian equation except the volume fraction equates to one and the interaction between the phases is not included. It can be mathematically written as: 


$$
\frac{\partial}{\partial t}(\rho \vec{v})+\nabla \cdot(\rho \vec{v} \vec{v})=-\nabla p+\nabla \cdot \overline{\bar{\tau}}+\rho \vec{g}+\vec{F}
$$

Where, $\vec{F}$ is an external force emerged due to an interaction with the discrete phase. Likewise, the momentum equation for the particles is based on the Newton $2^{\text {nd }}$ law of motion as shown below:

$$
\rho_{p} \frac{\mathrm{d} \overrightarrow{u_{P}}}{\mathrm{~d} t}=\overrightarrow{F_{d}}+\overrightarrow{F_{b}}+\vec{F}_{V M}+\vec{F}_{f p}+\vec{F}_{P P}
$$

where $\overrightarrow{F_{d}}, \overrightarrow{F_{b}}, \vec{F}_{V M}, \vec{F}_{f p}$, and $\vec{F}_{P P}$ stand for the drag force, buoyancy force, virtual mass force, fluidparticle interaction, and particle-particle interaction. The simulations for this study are performed by means of the Ansys FLUENT using the discrete phase model (DPM). For a gas-particle flow with the volumetric particle fraction less than 10\%, the DPM provides a good prediction of the flow field. For such a low volumetric fraction, the effect of particle pressure and viscous stresses due to particle are not considered [44]. Hence, the dominating in the Lagrangian frame is the drag force given by:

$$
\overrightarrow{F_{d}}=F_{D}\left(\vec{u}_{g}-\overrightarrow{u_{P}}\right)
$$

where $\vec{u}_{g}$ and $\overrightarrow{u_{P}}$ stand for the gas and particle velocity with $F_{D}$ being equivalent to:

$$
F_{D}=\frac{18 \mu C_{D} \mathrm{Re}}{24 d_{p}^{2}}
$$

The relative Reynolds number, Re in the above equation is defined as:

$$
\operatorname{Re}=\frac{\rho \mathrm{d}_{P}\left|\overrightarrow{u_{g}}-\overrightarrow{u_{p}}\right|}{\mu} \text {. }
$$

Also, the drag coefficient is given by

$$
C_{D}=\frac{24}{\operatorname{Re}}\left(1+b_{1} \operatorname{Re}^{b_{2}}\right)+\frac{b_{3} \operatorname{Re}}{b_{4}+\operatorname{Re}^{\prime}}
$$

where

$$
\begin{gathered}
b_{1}=\exp \left(2.3288-64581 \emptyset+2 \cdot 4486 \emptyset^{2}\right), \\
b_{2}=0.0964+0.5565 \emptyset
\end{gathered}
$$




$$
\begin{aligned}
& b_{3}=\exp \left(4.905-13.8944 \varnothing+18.4222 \emptyset^{2}-10.2599 \emptyset^{3}\right), \\
& b_{4}=\exp \left(1.4681+12.2584 \emptyset-20.7322 \emptyset^{2}+15.8855 \emptyset^{3}\right) .
\end{aligned}
$$

Here $\emptyset$ is the shape factor and is defined as

$$
\emptyset=\frac{S}{S},
$$

where $S$ is the actual surface area of the particle while $s$ is the surface area of the sphere having the same volume as the particle.

\subsection{Coupling Between Phases}

In a gas-solid flow, drag and turbulence of the gas phase always influence the solid or particle phase. However, a higher particle loading counter influences the carrier fluid phase. The DPM method available in the Ansys FLUENT provides the option to model the gas-solid flow for both scenarios. The DPM method has both the one-way coupling and two-way coupling options [44].

If a gas phase influences solid phase without any counter influence of solid phase on gas phase, then such interaction between the phases is defined as the one-way coupling while the presence of mutual influence of phases on each other is regarded as the two-way coupling. The coupling of the phases can occur through the mass, momentum, and energy transfer [45]. In the DPM method, the mass, momentum and energy (gained or lost by the particles) are calculated by means of computing the trajectory of the particles. The change of these quantities is incorporated into the continuous phase calculations, which appears as an interacting source term while two-way coupling is choose. Also in the case of two-way coupling, the effect of particle-phase trajectories is considered, which is accomplished by solving the equilibrium equations for the gaseous and the particle phase until the solution in both phases stops changing [46].

\subsection{Turbulence Dispersion}

The dispersion of the particles due to the mean and turbulent components of the fluid phase is known as turbulence dispersion [47]. The Ansys FLUENT offers two option for predicting the turbulence dispersion in a gas-solid flow, namely: 
1) The particle cloud model;

2) The Stochastic model/Random walk model.

The particle cloud tracking model utilizes the statistical method for calculating the turbulence dispersion of particles. The Gaussian probability density function (PDF) is used for representing the concentration of particles, whose variance is based on the degree of particle dispersion. The particle dispersion is caused by the turbulent fluctuations of the gas. The ensemble-averaged of the equation of particle motion yields the mean trajectory.

In the stochastic methods, the instantaneous equation for particle motion is solved. Instantaneous turbulent velocity is assumed to affect the particle trajectory [48]. Usually, when the turbulence dispersion is not included, Eq. (3.36) is solved using just mean velocity component $\bar{u}$. However, for the prediction of particle dispersion, the fluctuating component, $u^{\prime}$, is also added, and hence, the equation for the motion of the particles is solved using the instantaneous velocity

$$
u=\bar{u}+u^{\prime}
$$

In the discrete random walk model (DRW)/eddy lifetime mode, the fluctuating velocity is assumed to be a discrete function of time. The particles are assumed to be interacting with the eddies, which are characterized by a Gaussian-distributed velocity fluctuation and a time scale. The random fluctuating velocity is formulated as:

$$
u^{\prime}=\zeta \sqrt{\overline{u^{\prime 2}}}
$$

where $\zeta$ is the normally distributed random number. For a particular turbulence model, the turbulent kinetic energy is calculated, and hence the root mean square (rms) velocity $\sqrt{\overline{u^{\prime 2}}}$ reads:

$$
\sqrt{\overline{u^{\prime 2}}}=\sqrt{\frac{2 k}{3}}
$$




\section{Methodology}

Detailed numerical study of the gas-solid phases flow in an annular geometry with uniform crosssection is presented in this thesis. The primary aim of this research is to get a vivid understanding of the gas-particle flow field inside the secondary inlet section of the SPOC geometry, which is believed to have a significant role in defining the flame structure inside the combustor. The CFD simulations are performed using Ansys FLUENT.

\subsection{Geometry and Domain Discretization}

This study is focused on the secondary inlet of the SPOC geometry. Some changes have however been made. Specifically, a hollow cylinder with an annular structure and uniform cross-section is used for this study, while the secondary inlet of the SPOC had the converging cross-section. Convergence in geometry is likely to accelerate the flow hence, uniform cross-section structure is used here to eliminate the influence of convergence in the flow field. The study is solely focused on the fundamental behavior of fluid-particle flow in the annular cylinder at an elevated pressure. The Ansys FLUENT employs the control-volume technique for solving the governing equations, namely, that of continuity, momentum, and energy for the fluid flow. A computational grid is first generated to divide the domain into a number of the discrete control volumes. To construct an algebraic equation for the discrete, variables such as the velocities and pressure, the integration of the governing equations is performed on the individual control volumes. Finally, the set of discretized equations and the solution of the resulting equations are linearized to obtain the updated values of the dependent variables. An efficient and reliable meshing software, ICEM, was used for designing geometry and generating the mesh.

Figures 4.1 and 4.2 show the wireframe and solid structure of the geometry, which possesses the inlet section, the outer wall, the inner wall, and the outlet section - depicted in Fig. 4.1 by blue, green, red, and yellow regions, respectively. Further, Figs. 4.3 and 4.4 delineate geometry without the outer wall, while Fig. 4.5 shows only the inlet section. The total length of the domain is $0.2 \mathrm{~m}$, 
with the inner and outer radii of 4.7 and $14.2 \mathrm{~mm}$, respectively. The mesh contains 62,720 nodes with 336 mass inlet flow face. The details of various geometry dimensions are shown in Table 1.

Table 1: Domain extents for the geometry used in the simulation.

\begin{tabular}{|l|l|l|l|}
\hline \multicolumn{3}{|c|}{ Domain Extents (m) } \\
\hline Xmin & -0.2032 & Xmax & 0 \\
\hline Ymin & -0.0142 & $Y \max$ & 0.0142 \\
\hline Zmin & -0.0142 & Zmax & 0.0142 \\
\hline
\end{tabular}

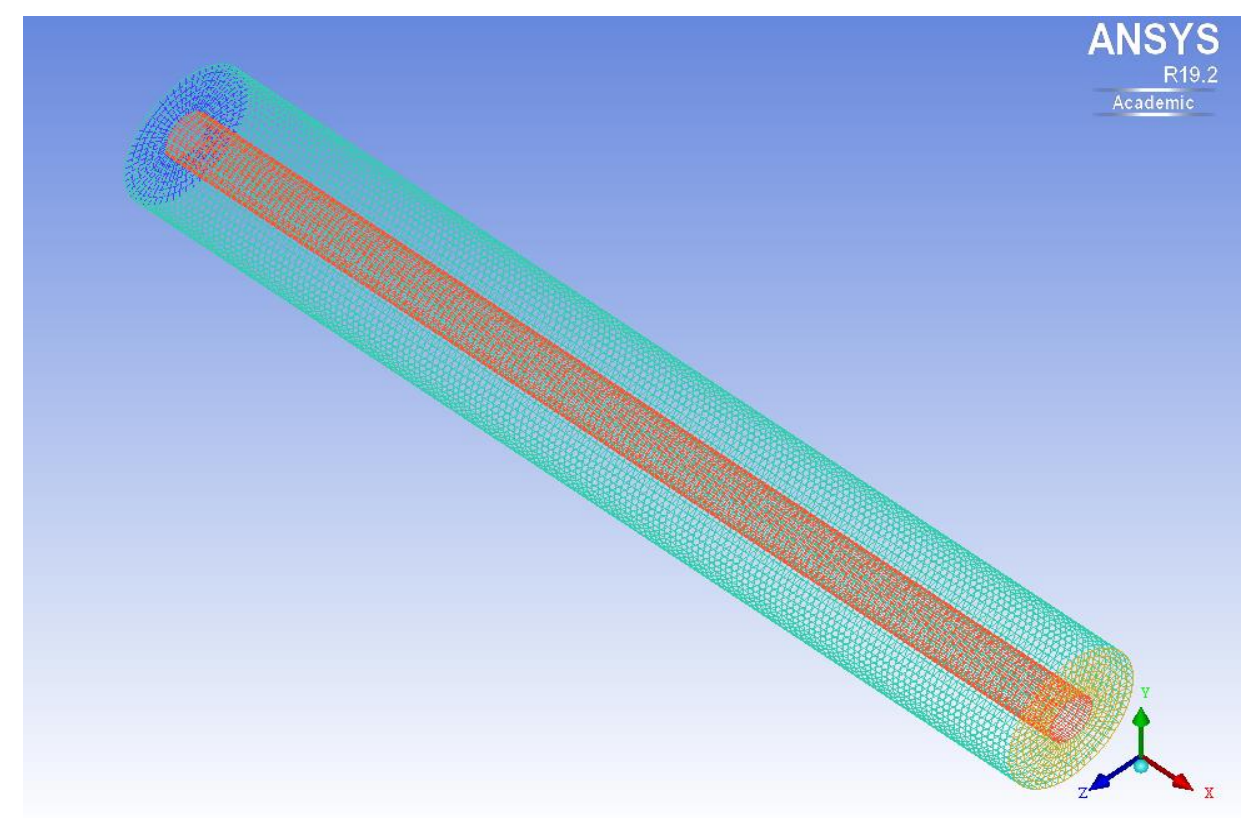

Figure 4.1: A wireframe structure of the configuration used. 


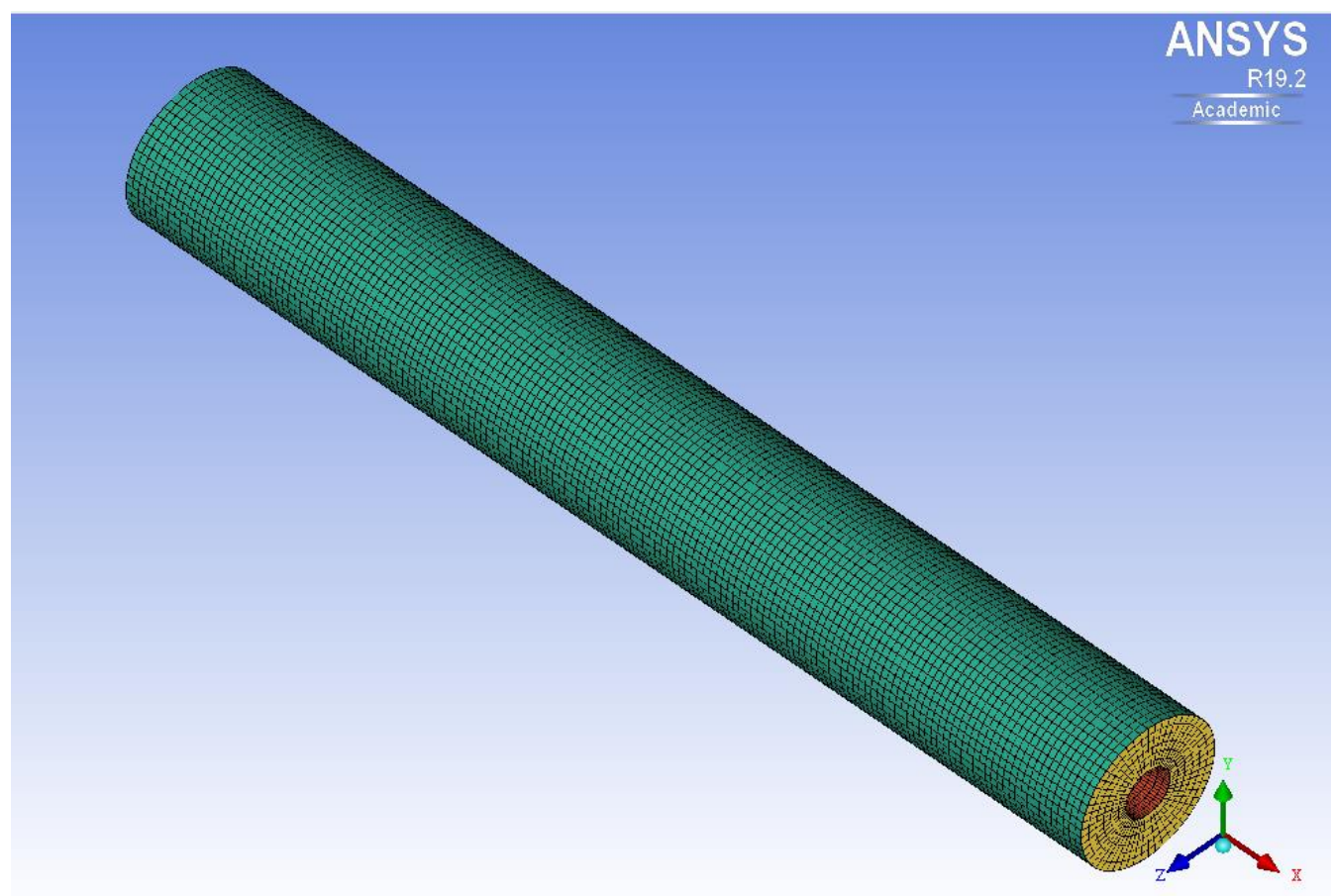

Figure 4.2: The solid structure of the configuration used.

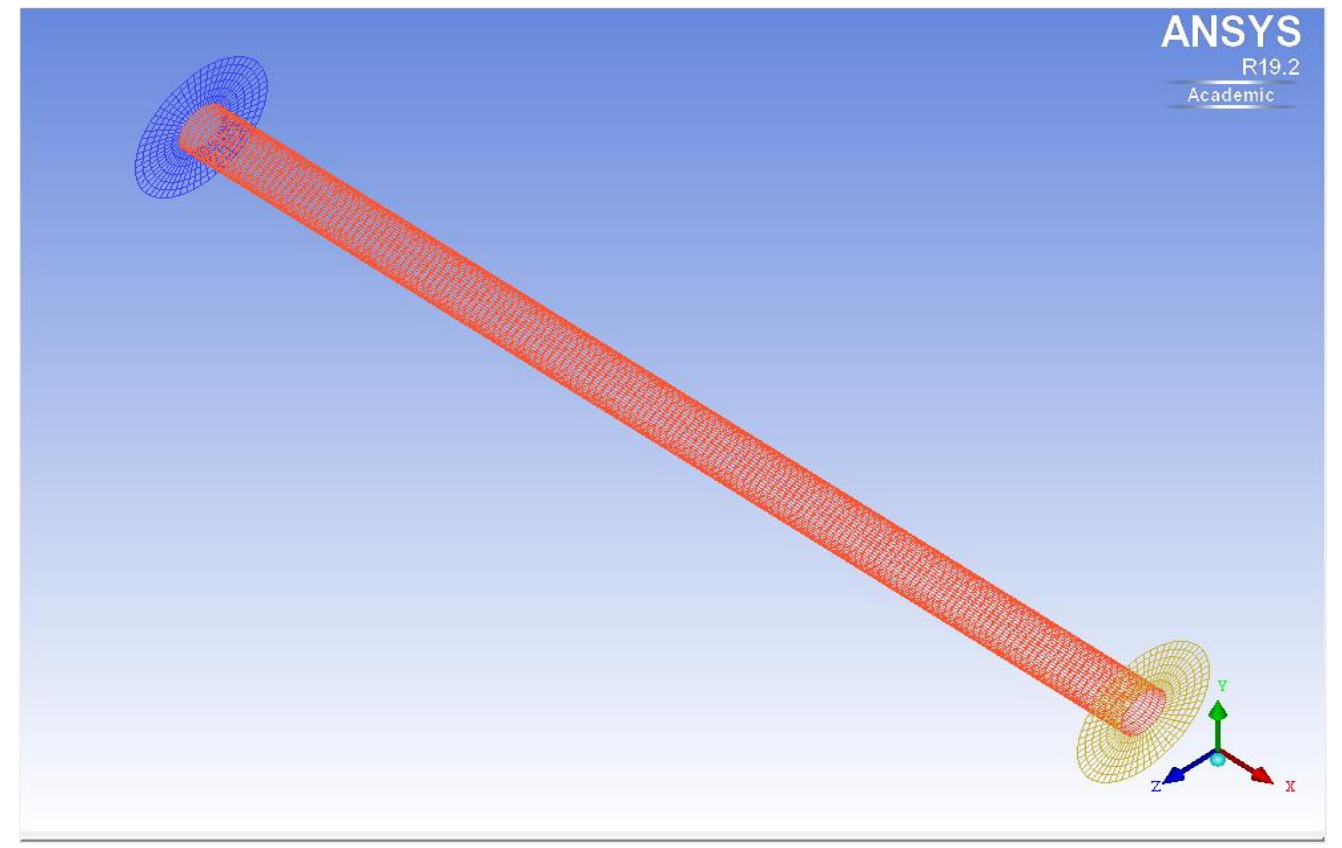

Figure 4.3: The section of the geometry with inlet, outlet, and inner wall. 


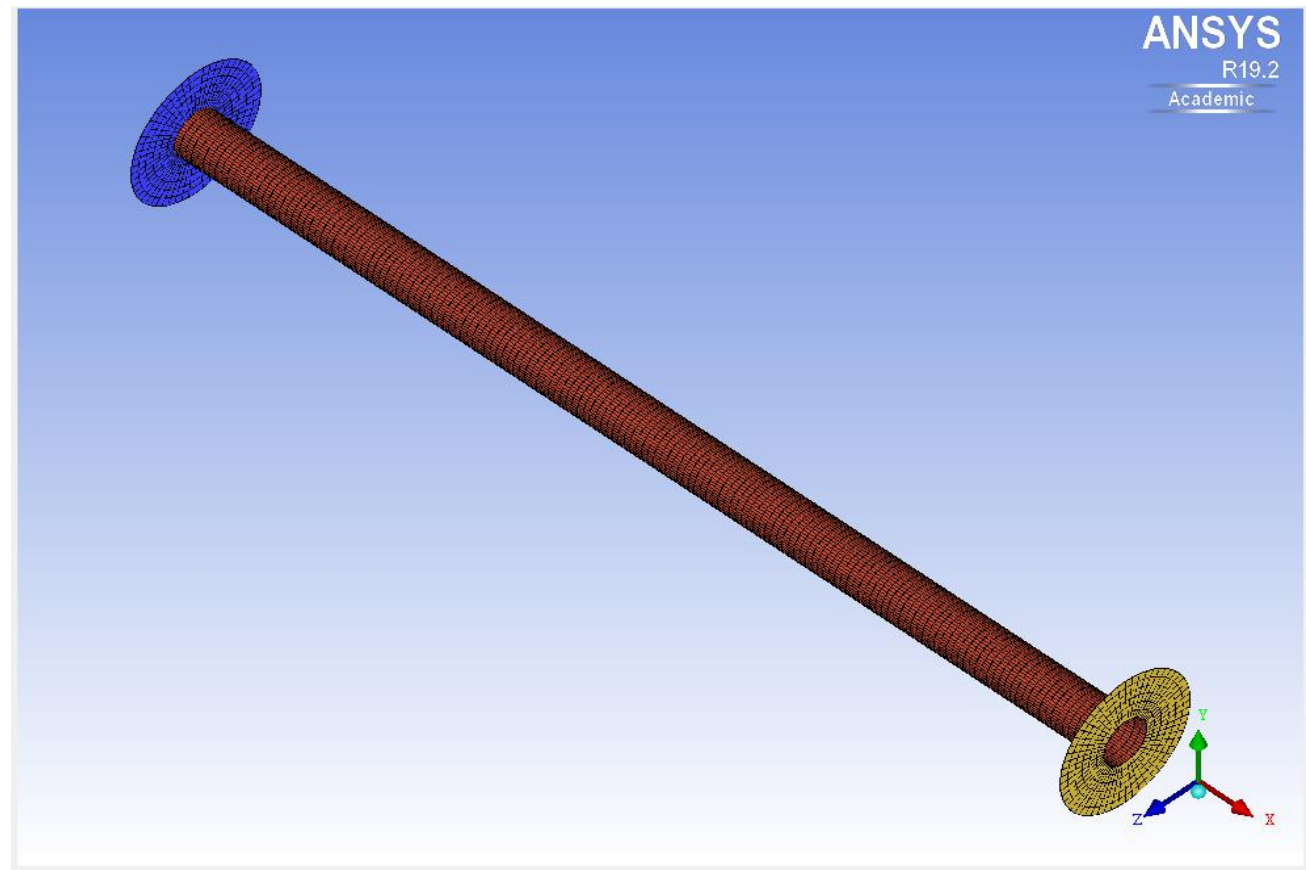

Figure 4.4: The solid structure of the geometry with inlet, outlet, and inner wall.
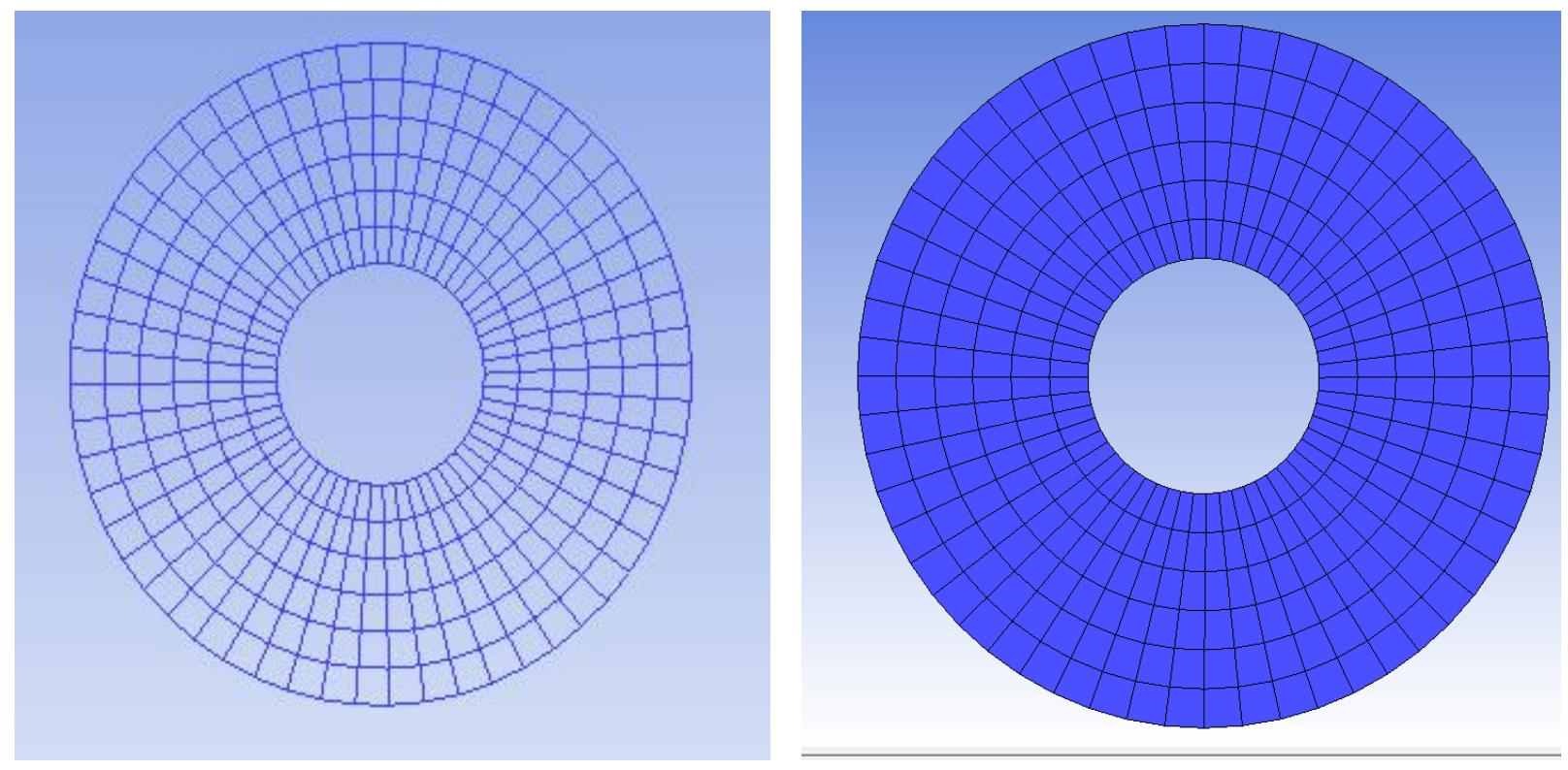

Figure 4.5: The Wireframe and solid structure of inlet surface (particles injection surface). 


\subsection{Numerical Method and Boundary Conditions}

Transient RANS simulations were performed for a pure gas flow as well as for a gas-solid phase flow. The $k-\omega$ SST model was particularly selected to capture the flow at the vicinity and away from the wall, i.e. at the free stream. The injection of particle was done based on flow through time (FFT) which is the time required by the fluid to cross the domain. The simulation was first performed to generate the steady transient solution which was followed by the injection of the particles at 5FFT. A gas-solid, two-phase flow process is modeled using the EL approach. The fluid/gas phase was modeled using the Eulerian approach while the Lagrangian method was employed for modeling solid/particles. The coupling between the phases was done through the source term in the governing equation. Particularly the DPM was selected for this purpose. Both one-way and two-way couplings were studied. The unsteady particle tracking was done while a stream or the parcels of particles was injected at the fluid flow time steps of $10^{-3} \mathrm{~s}$. Originally, for the SPOC process, the particles obeying the Rossin-Rammler (RR) size distribution were injected. However, to exclude the problem of the flow stability, which was presumably to instigate due to non-uniform size distribution, the particles with a fixed size of 65 microns were injected from the inlet surface. For a given mass flow rate of the particle, the RR diameter distribution divides the particles into several discrete intervals. However, a uniform diameter distribution injects the particle with a size of single diameter. The analysis was performed with and without consideration of the effect of turbulence dispersion of the particles. This effect has been accounted by means of the discrete random walk model. Two categories of the particle flow rates were used. Specifically, the mass flow rate of particles, identical to that in the SPOC process, was defined as high particle loading. In addition, to study the influence of the particle loading, the mass flow rate was decreased by an order of magnitude and was referred to as low particle loading.

Spatial discretization for the scaler parameters was performed using both the $2^{\text {nd }}$-order upwind and the $3^{\text {rd }}$-order monotone upstream-centered schemes for conservation laws (MUSCL). However, no difference was observed. The transient formulation was done using a bounded $2^{\text {nd }}$-order implicit method. The vector parameters such as momentum were spatially discretized using a $2^{\text {nd }}$-order upwind method. For the pressure-velocity coupling, the semi-implicit method for pressure-linked 
equation (SIMLE) method was used. The operating pressure of the system was identical to that of the SPOC, being as elevated as 15 bar. Various boundary conditions are listed in Table 2.

Table 2: Boundary conditions used in the simulation.

\begin{tabular}{|l|l|}
\hline Parameters & Values \\
\hline Inlet mass flow rate of air & $2.45 \mathrm{~g} / \mathrm{s}$ \\
\hline Inlet mass flow rate of particles & $3.98 \mathrm{~g} / \mathrm{s}$ and $0.39 \mathrm{~g} / \mathrm{s}$ \\
\hline Size of injected particles & $65 \mu \mathrm{m}$ \\
\hline Wall boundary condition & No-slip \\
\hline
\end{tabular}




\section{Result and Discussion}

In this section, the results obtained from the numerical investigation of the impact of particle injection on the gaseous flow at elevated pressure are presented. The effects of various parameters, the coupling between phases, particle loading, and turbulence dispersion of particles, are shown in detail. Based on the volumetric fraction of the particles in the flow, the particle loading has been categorized as (i) high particle loading [that with the particle mass flow rate of $3.98 \mathrm{~g} / \mathrm{s}$ and the volumetric fraction of $1.2 \%$ ] and (ii) low particle loading [that with the particle mass flow rate of $0.39 \mathrm{~g} / \mathrm{s}$ and the volumetric fraction of just $0.13 \%$ ]. Further, the simulations have been performed with both one- and two-way coupling/interaction between the phases. Also, an investigation has been carried out for understanding the role of turbulence dispersion of the particle in the gas phase.

\subsection{Verification of Mass Conservation}

After the particles were injected into the gas flow, the DPM concentration contours were inspected. For both converging and uniform cross-section geometries, concentration gradient was observed: see the blue and green zones in Figs. 5.1 and 5.2. Therefore, an investigation to validate the conservation of a particle number was performed. The entire flow domain was treated as a control volume, where the particles entered from the inlet surface and exited from the outlet one. Since the mass flow rate of the particles was known, the mass of the particle and, hence, the number of particles entering and exiting from the control volume was calculated as

$$
\begin{gathered}
\dot{M}=M / \Delta t, \\
M=N_{p} \times \forall \times \rho_{p}, \\
N_{p}=\frac{M}{\forall \times \rho_{p}} .
\end{gathered}
$$

Here, $\dot{M}$ is a mass flow rate of the particles, $M$ is a mass of the total number of particles entering the control volume at each injection time step, and $N_{p}$ is the number of particles enters through the inlet during each injection. Each particle has a volume $\forall$ and density $\rho_{p}$. The mass flow rate of the particles versus time steps at the inlet and outlet section is shown in Fig. 5.3 and 5.4 respectively. 
The difference between the number of particles entering and exiting the domain is shown in Fig. 5.5 .

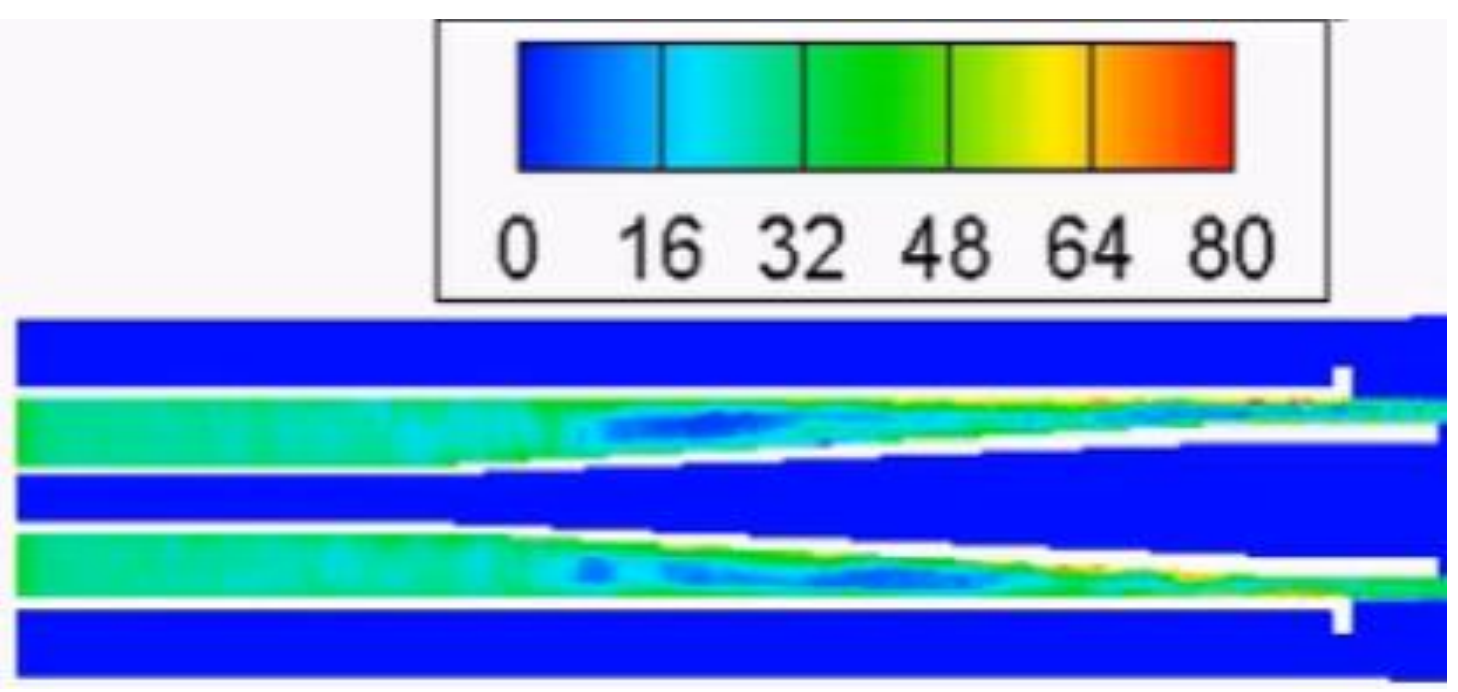

Figure 5.1: A contour of the DPM concentration in the SPOC process [23].

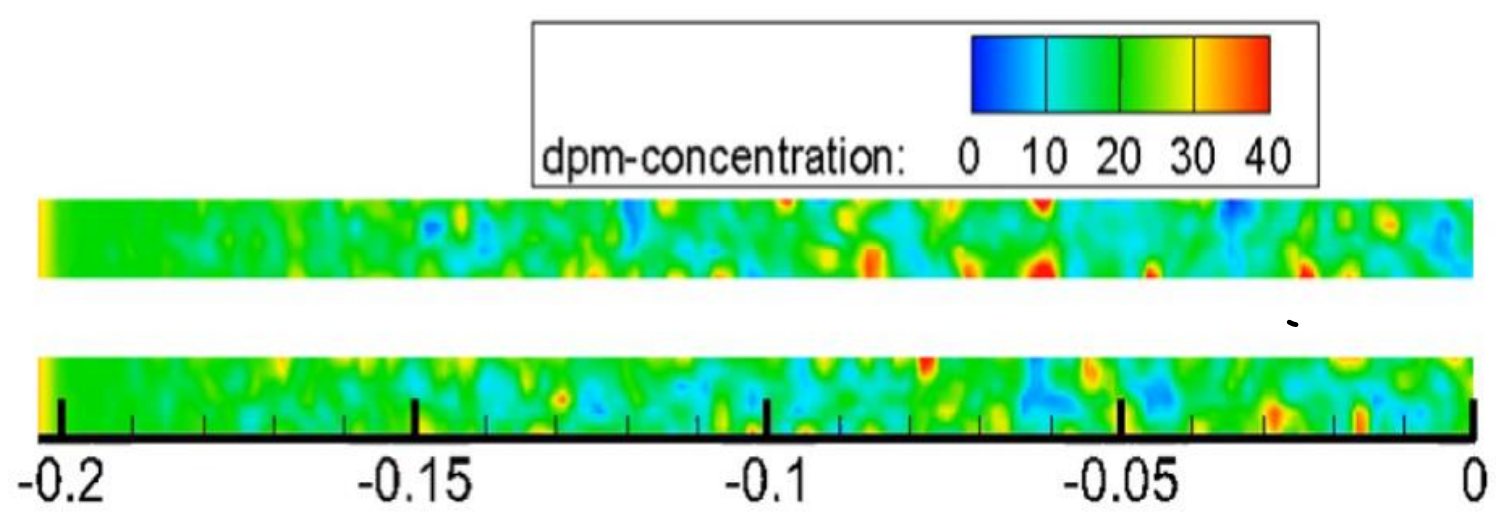

Figure 5.2: A contour of the DPM concentration. 


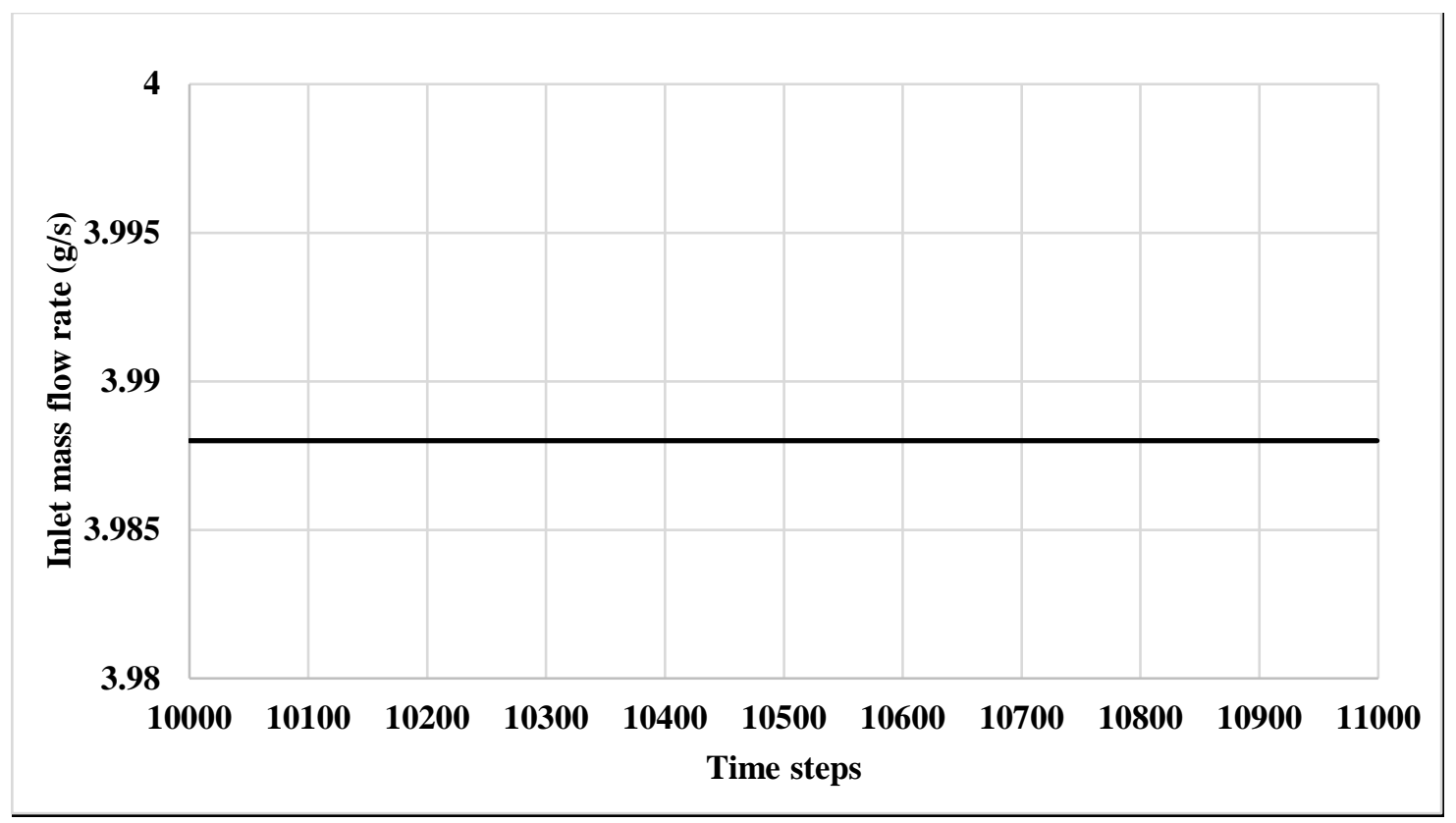

Figure 5.3: The particle mass flow rate at the inlet versus time.

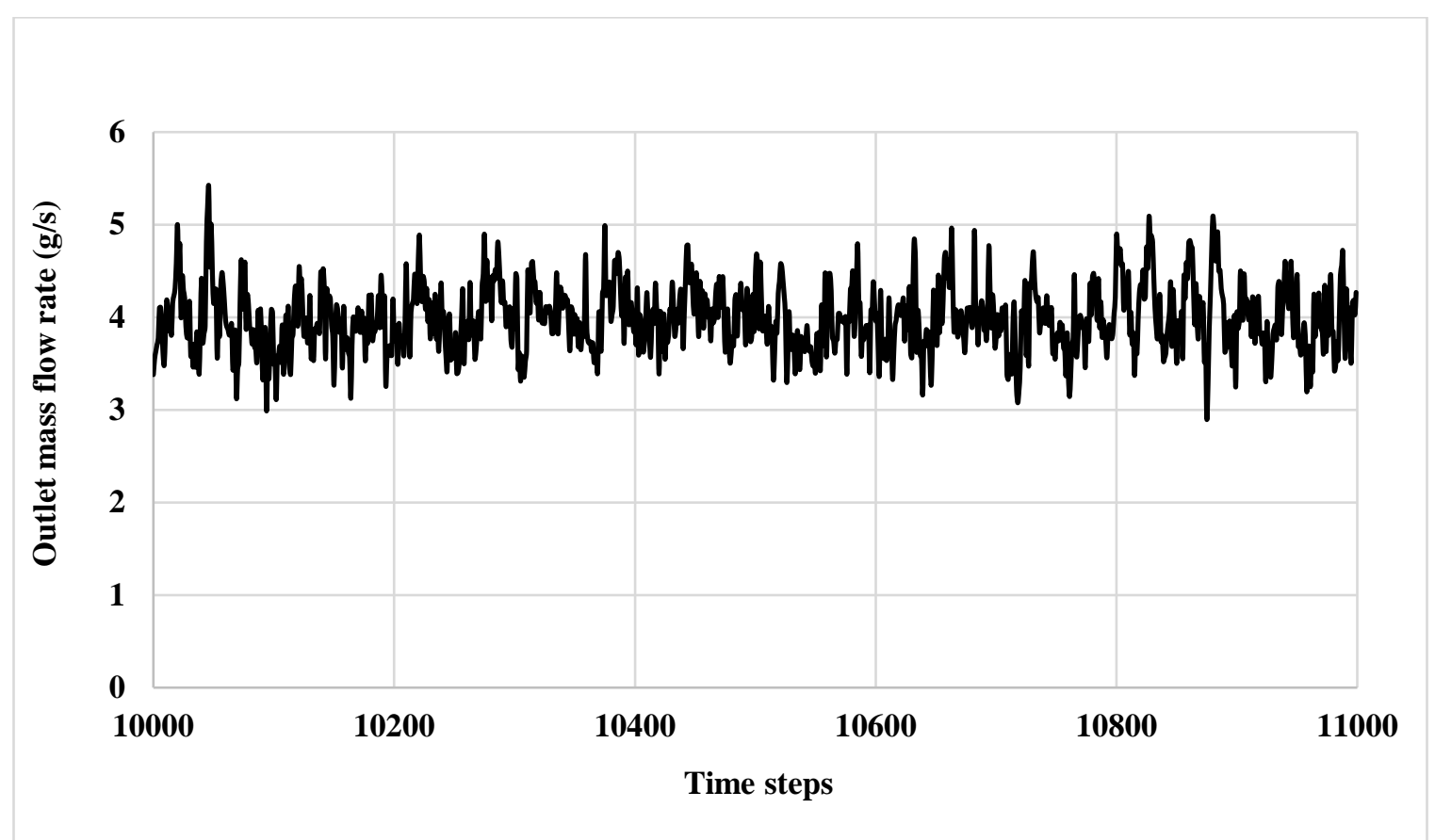

Figure 5.4: The particle mass flow rate versus time at the outlet. 


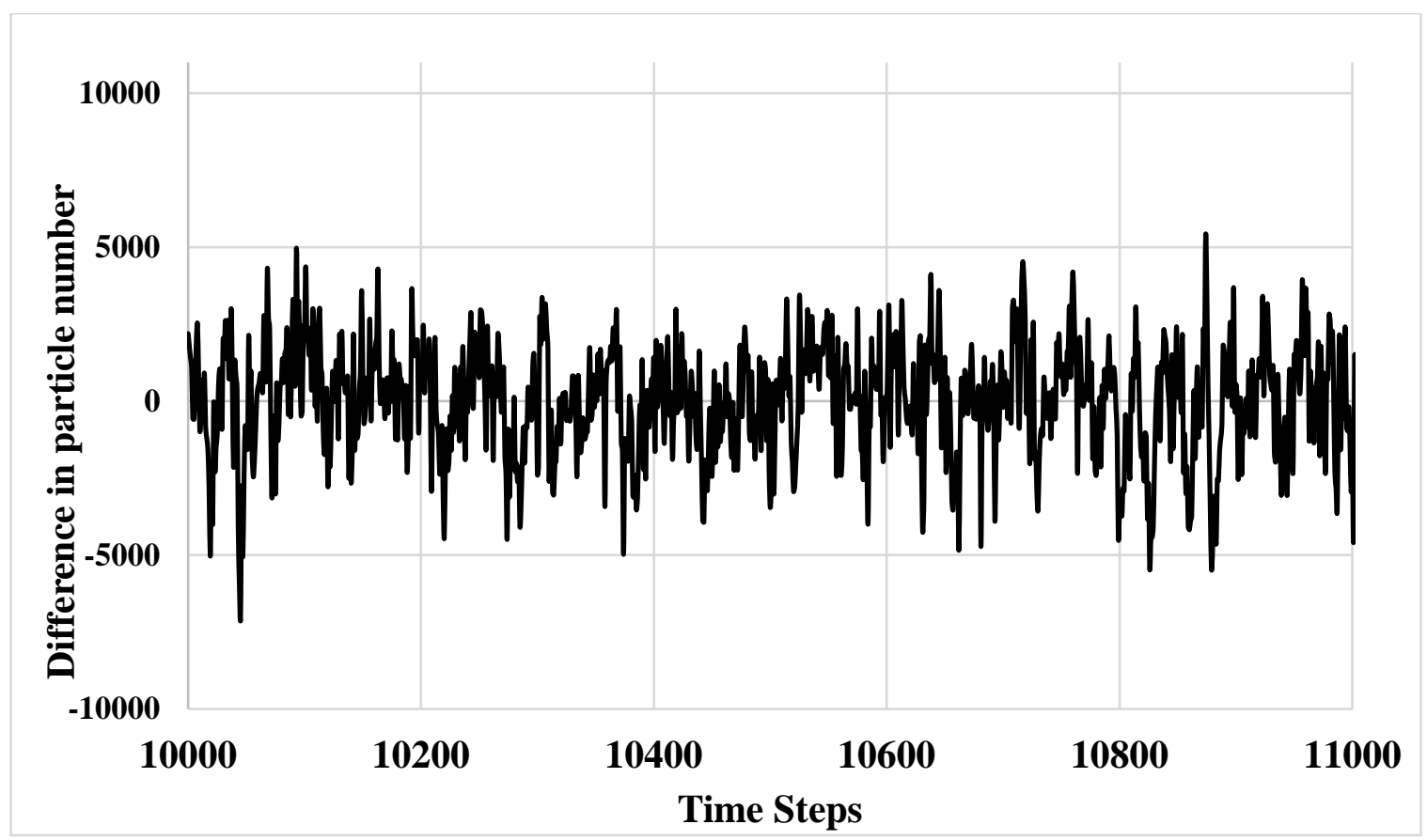

Figure 5.5: Difference in particle number entering and exiting from the control volume versus time.

Figures 5.3 and 5.4 depict the particles mass flow rate at the inlet and outlet of the control volume at various time steps. Uniform injection of the mass flow rate is seen at the inlet. However, the mass flow rate at the outlet is erratic and non-uniform. Specifically, the mass flow rate at the outlet exceeds that at the inlet at some random time, while it is smaller at other times. Likewise, the difference in the particle number at the inlet and the outlet sections, shown in Fig.5.5, is sometimes positive while sometimes it is negative. However, their mean value is zero. Therefore, it was found that, despite the uniform injection of the particle at the inlet, the particles coming out of the domain are non-uniform. Also, the number of particles is conserved, which justifies the clustering of the particles inside the control volume.

\subsection{One-way and Two-way Coupling with and without Turbulence Dispersion}

Seven different cases with various combinations between the phase-coupling and turbulence dispersion have been developed and studied as summarized in Table 3. The volume fraction of the particles in the flow has been calculated analytically to validate the computational result obtained with the Ansys FLUENT. 
Table 3: Seven different cases developed to study phase coupling, turbulence dispersion, and particle loading.

\begin{tabular}{|l|c|c|c|c|}
\hline Cases & Phases & Particle loading & Coupling & Turbulence dispersion \\
\hline $\mathbf{1}$ & Pure gas phase & No particle & No coupling & off \\
\hline $\mathbf{2}$ & Two-phase & Low & One way & on \\
\hline $\mathbf{3}$ & Two-phase & Low & Two way & off \\
\hline $\mathbf{4}$ & Two-phase & Low & Two - way & on \\
\hline $\mathbf{5}$ & Two-phase & High & One way & off \\
\hline $\mathbf{6}$ & Two-phase & High & Two - way & on \\
\hline $\mathbf{7}$ & Two-phase & High & Two - way & \\
\hline
\end{tabular}

The volumetric fraction $V_{F}$ of the particles is defined as the ratio between the total volume of the particles and the volume of the domain. Since in the present study, the volume of the domain is the summation of the volume of total particles and the volume of gas $V_{g}$, the volume fraction reads

$$
V_{F}=\frac{V_{P}}{\left(V_{P}+V_{g}\right)} .
$$

From the mass flow rate and the cross-section area of the inlet, the volume of the gas was calculated to be $1.14 \times 10^{-4} \mathrm{~m}^{3}$. Likewise, the volume occupied by the particles for high particle loading was $1.48 \times 10^{-6} \mathrm{~m}^{3}$, which corresponds to the volumetric fraction of the particles of $1.2 \%$ : the same as that obtained from the FLUENT. For the low particle loading case, the volume fraction was $0.13 \%$.

\subsubsection{Case 1: Pure Gas Flow}

First, the simulation was initiated with a pure gas (air) flow into the system. Hence, this was purely single-phase problem. The axial velocity at the four different locations were plotted, namely: the inlet, $x=-0.1 \mathrm{~m}, x=-0.05 \mathrm{~m}$, and the outlet. The locations for $x=-0.1 \mathrm{~m}$ and $x=-0.05 \mathrm{~m}$ are shown by the two vertical black lines in Fig. 5.6.

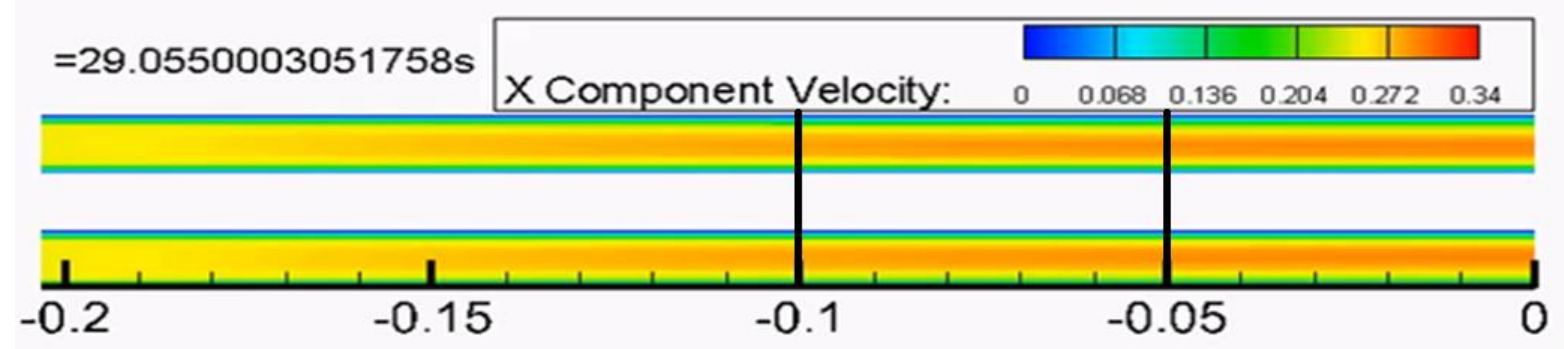

Figure 5.6: Axial velocity contour for a pure gas flow. The vertical black line shows the monitoring position. 
The graphs of the axial velocity versus the radial distance, Fig. 5.7, drawn at various axial locations show the developed flow field. However, no sign of turbulence is seen. The axial velocity of the gas phase is found to be growing as the velocity at the inlet is about $0.25 \mathrm{~m} / \mathrm{s}$ while it is $0.3 \mathrm{~m} / \mathrm{s}$ at the outlet.
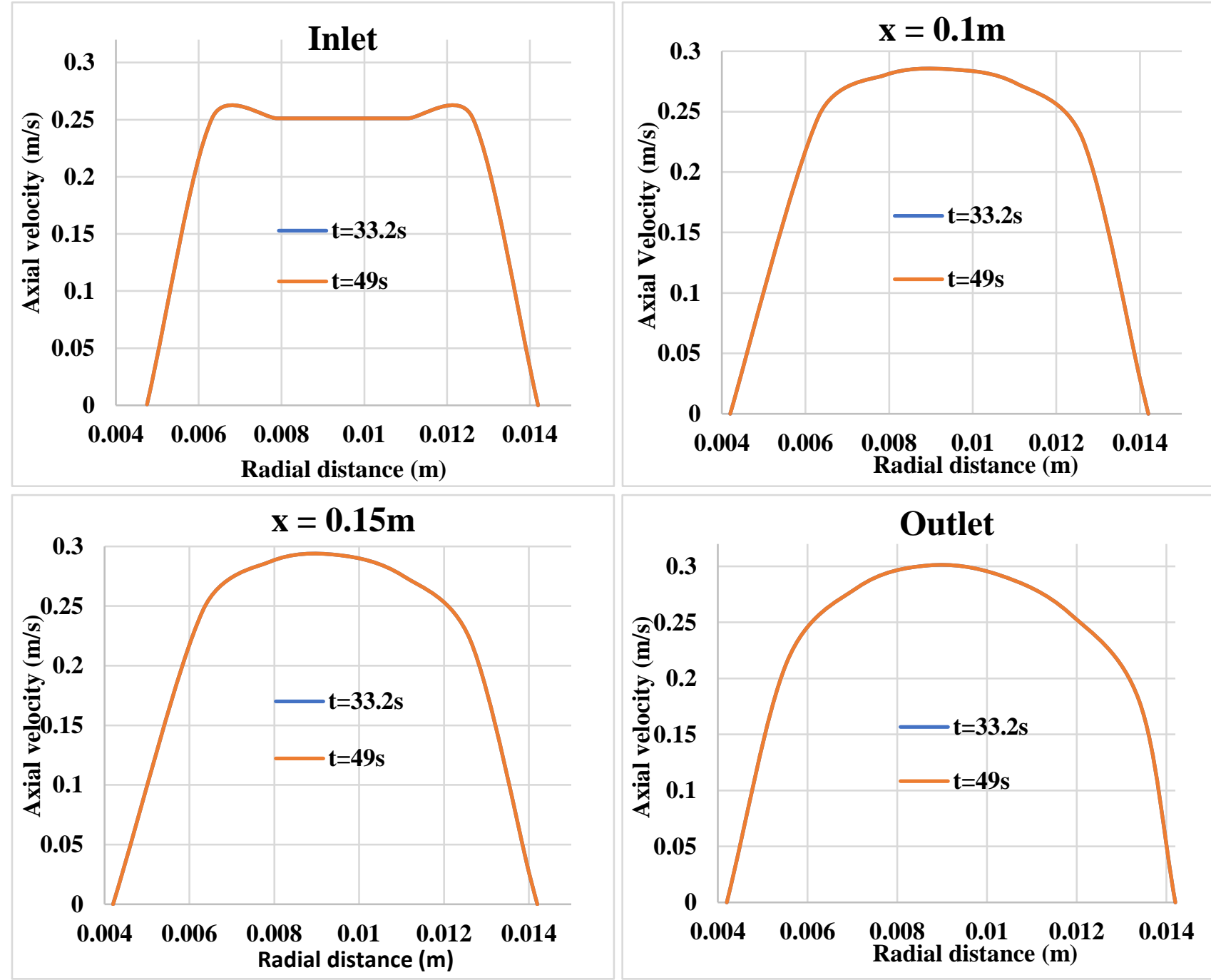

Figure 5.7: Axial velocity of the gas versus radial distance at various axial locations. 
Moreover, a horizontal line lying inside the boundary layer and in the vicinity of the wall was created. The specific turbulent kinetic energy (denoted as TKE in graphs) and the axial velocity along the horizontal line were monitored to see the flow field fluctuations. The plots for the TKE and the axial velocity along the horizontal line are shown in Figs. 5.8 and 5.9.

No fluctuations in the velocity and TKE profile is seen. Initially, the gas phase is injected with the desired velocity due to which the TKE is growing nevertheless, after a certain distance its value is falling and reach saturation. Hence, it was found that the instability in the flow field wasn't inherent in the gas phase but appears as a result of the particle injection.

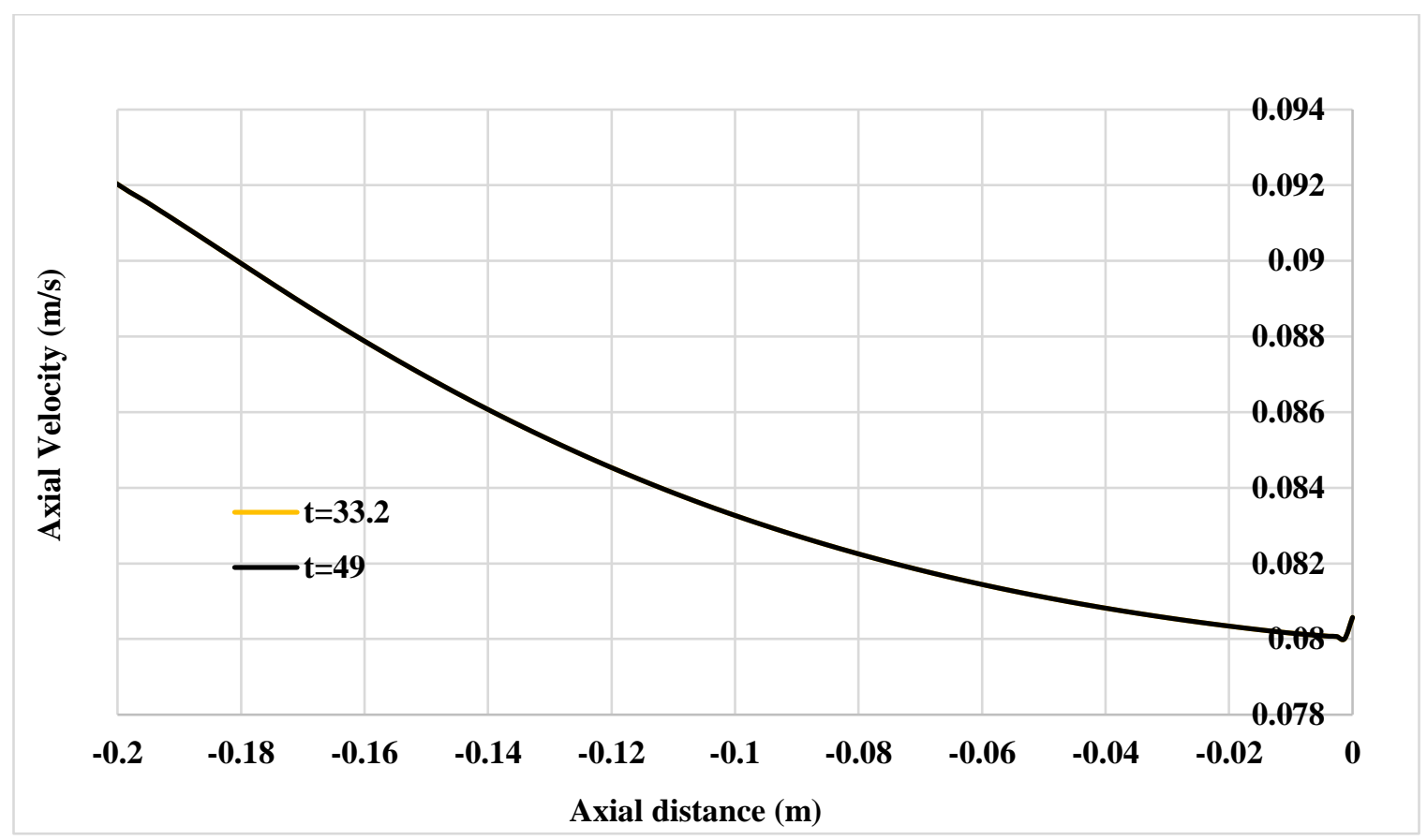

Figure 5.8: Axial velocity along with the boundary layer for a pure gas-phase flow 


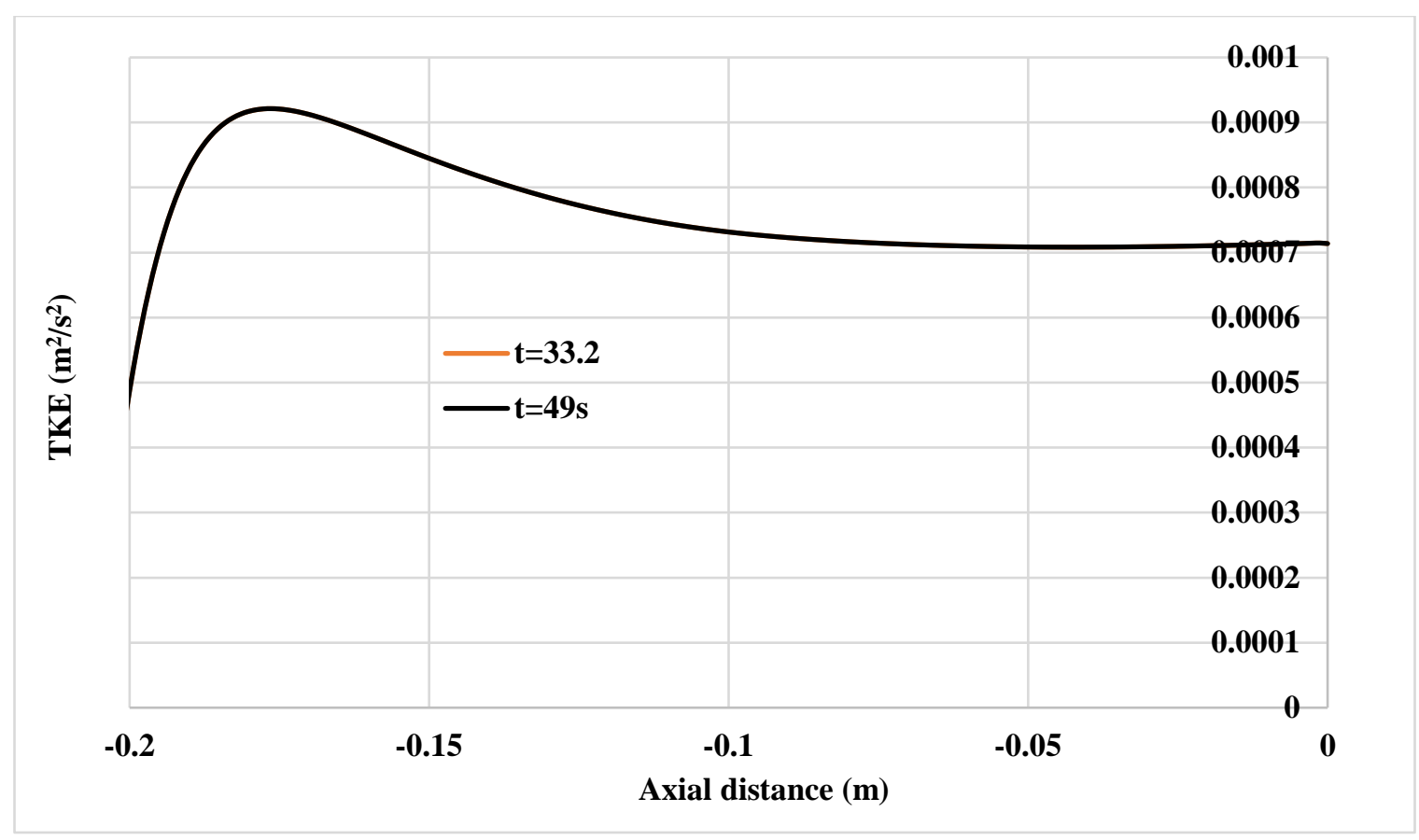

Figure 5.9: The TKE along with the boundary layer for a pure gas-phase flow.

\subsubsection{Influence of Gravity in a Flow}

The axial velocity, with which both the phases at the inlet boundary were injected, was the same (about $0.25 \mathrm{~m} / \mathrm{s}$ ). However, once both phases entered the domain, the difference in the velocity was observed immediately. The particles and gas velocities at two different locations are shown in Figs. 5.10 and 5.11. It can be seen that the change in the gas velocity is not large. However, there is a significant change in the particle's velocity. Gravity acting along the axial ( $x$-) direction was suspected to be the cause for such a velocity lag between the phases. To validate this hypothesis, a simulation was performed without gravity and the results obtained are shown in Figs. 5.12 and 5.13. Observing the graphs at inlet (Figs. 5.10 and 5.12) and outlet (Figs. 5.11 and 5.13), gravity was found to cause the velocity discrepancy between the phases. 


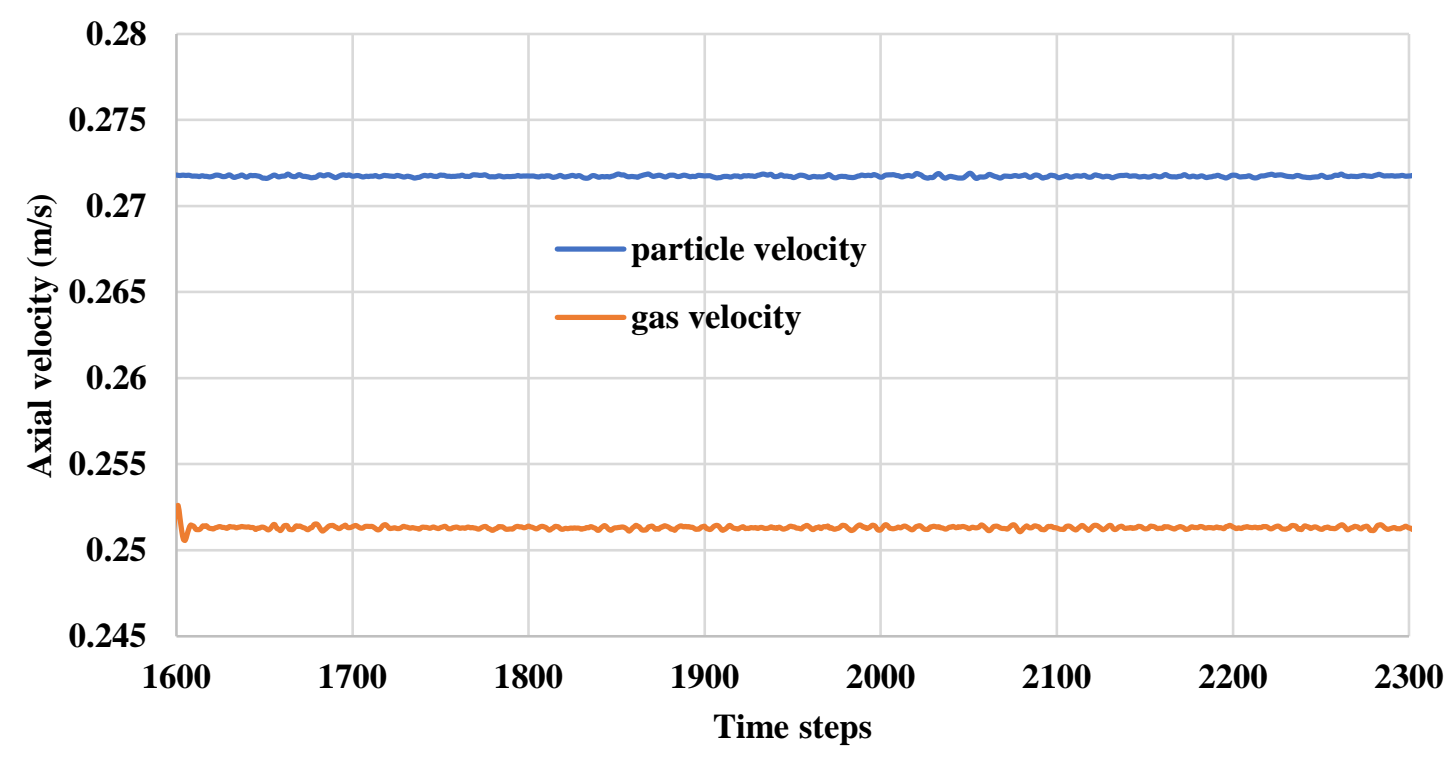

Figure 5.10: The axial velocity of the gas and the particles at the inlet in the presence of gravity.

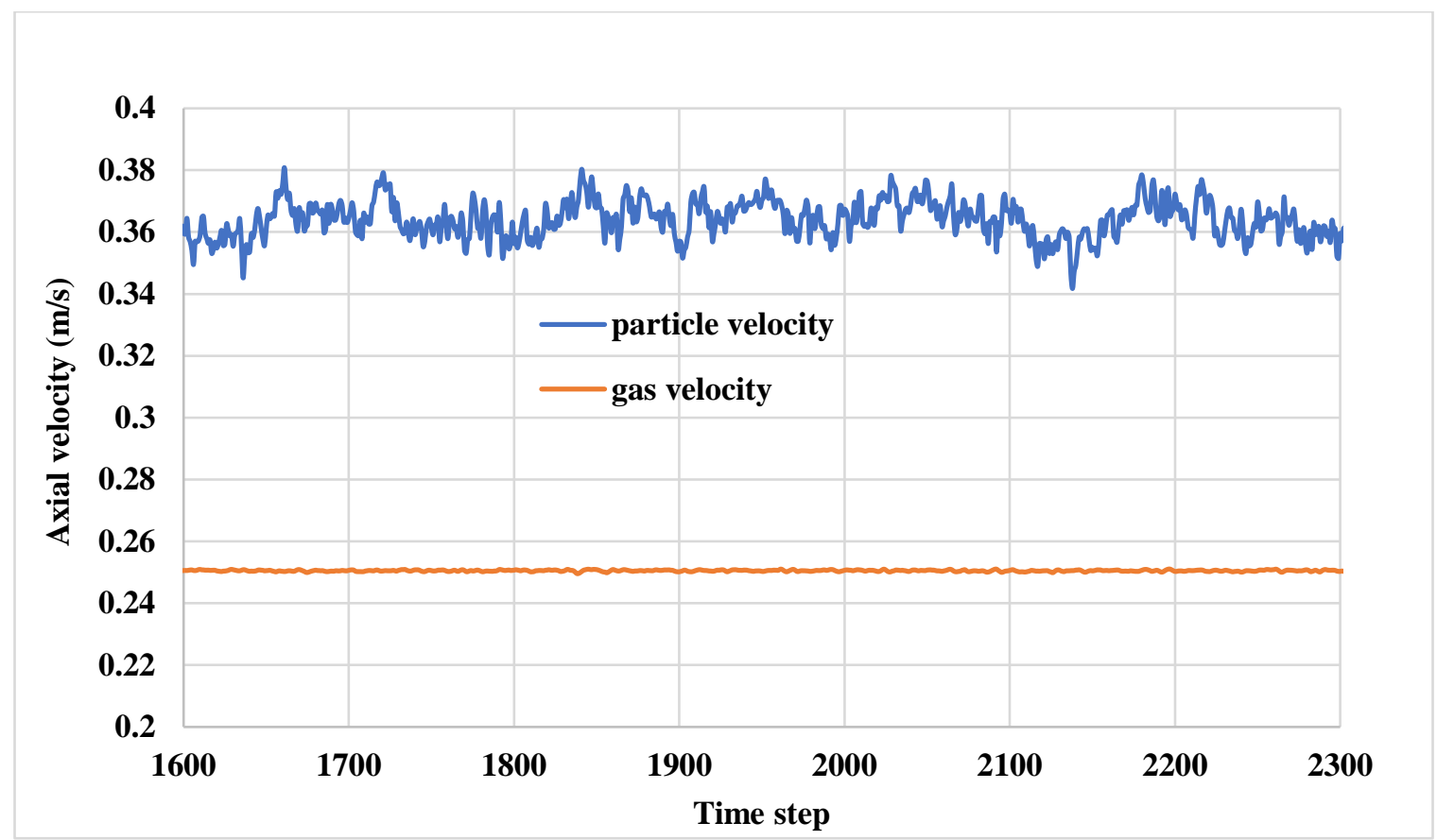

Figure 5.11: The axial velocity of the gas and particles at the outlet in the presence of gravity. 


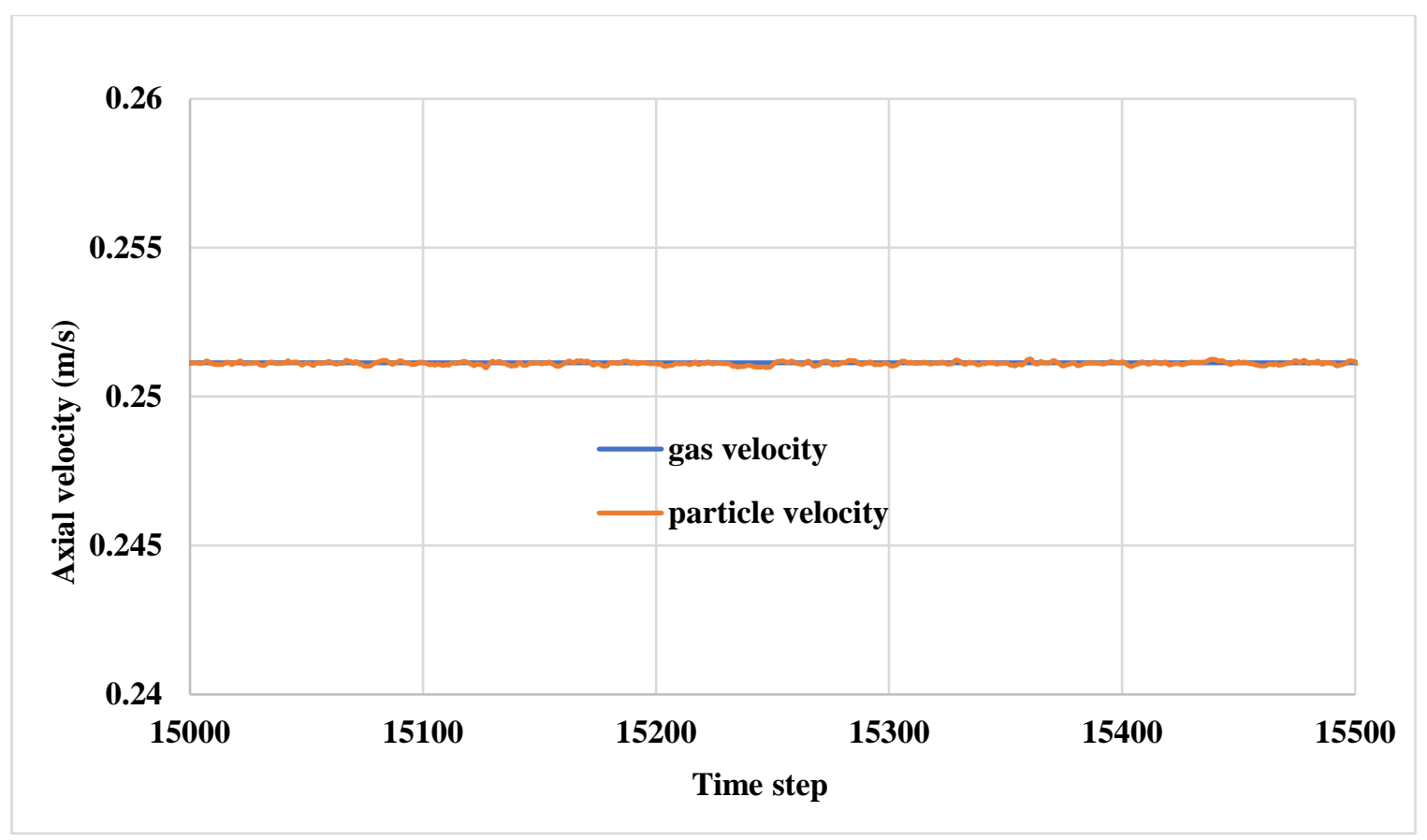

Figure 5.12: The axial velocity of the gas and particles at the inlet in the absence of gravity.

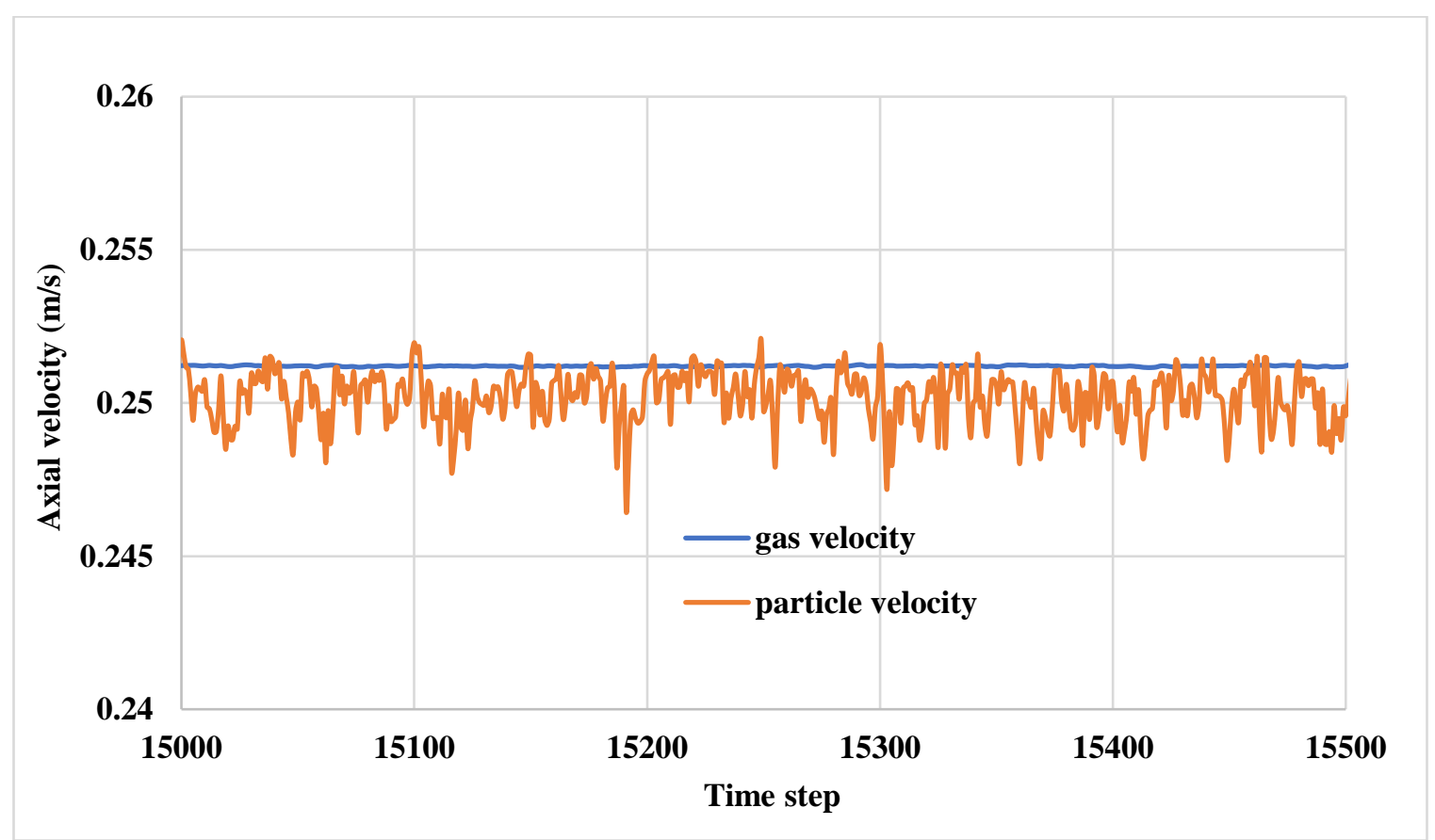

Figure 5.13: The axial velocity of the gas and particles at the outlet in the absence of gravity. 


\subsubsection{Low and High Particle Loading}

The three cases, namely, Cases 2, 3, and 4 of Table 3, were simulated. Case 2 had low particle loading with the inclusion of turbulence dispersion but had no coupling between the phase while Case 3 had low particle loading and two-way coupling, but the effect of turbulence dispersion of particle was neglected. Further, Case 4 combined the low particle loading with two-way coupling and turbulence dispersion. Similarly, these cases were repeated with high particle loading and are referred to as Cases 5, 6, and 7, respectively. The results obtained from each case are shown below.

\subsubsection{Case 2: Low particle loading with turbulence dispersion and one-way coupling}

Monitoring the axial velocities at the various axial location did not show any turbulence in the flow. In addition, the TKE plotted along the boundary layer did not show any fluctuations. The flow did not differ from a pure gas-phase flow. For a low volumetric fraction of injected particles, we did not observe any sign of clustering or instability in the flow field.

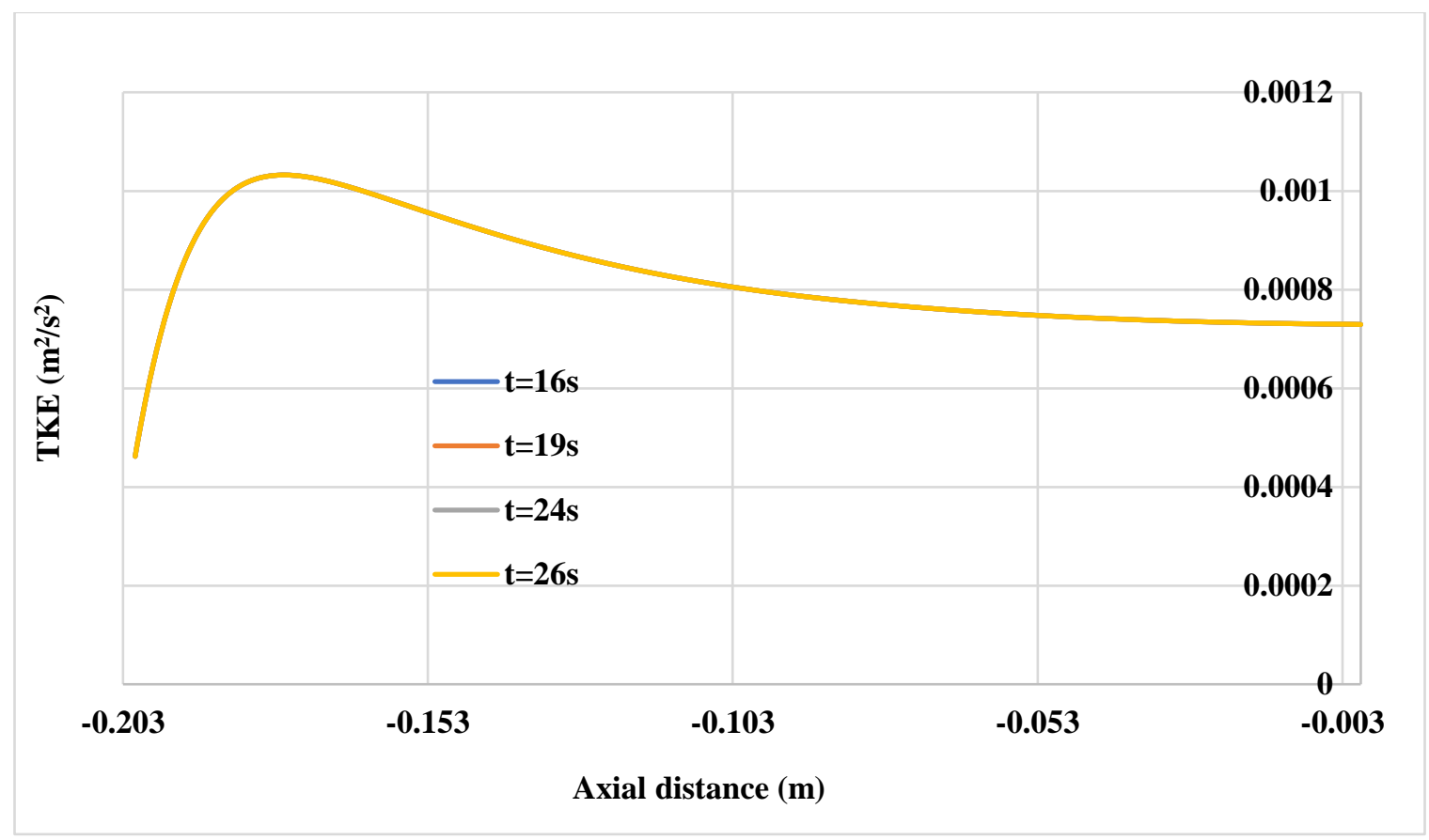

Figure 5.14: The TKE along the boundary layer for Case 2. 

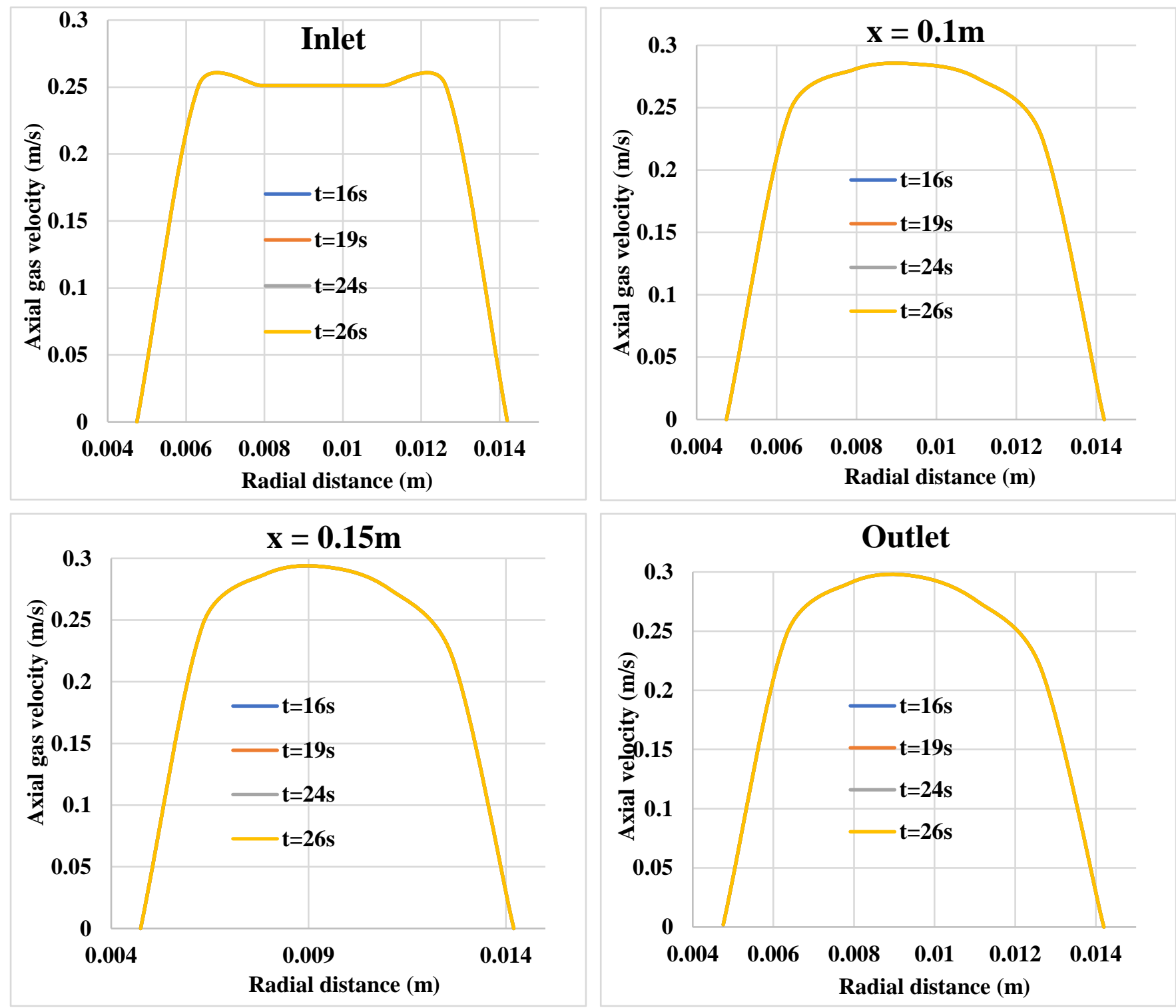

Figure 5.15: Axial velocity of the gas versus radial distance at various axial locations.

\subsubsection{Case 3: Low particle loading with no turbulence dispersion but two-way coupling}

Similar to the previous case, the axial velocity and the TKE were monitored at various locations at various time. Again, the axial velocity of the gas was found to be growing (Fig. 5.16) but was not as high as in Case 1. Since the velocity profile at different locations obtained for various times 
showed no distortion in the flow field, the results from this case did not deviate from the previous case.
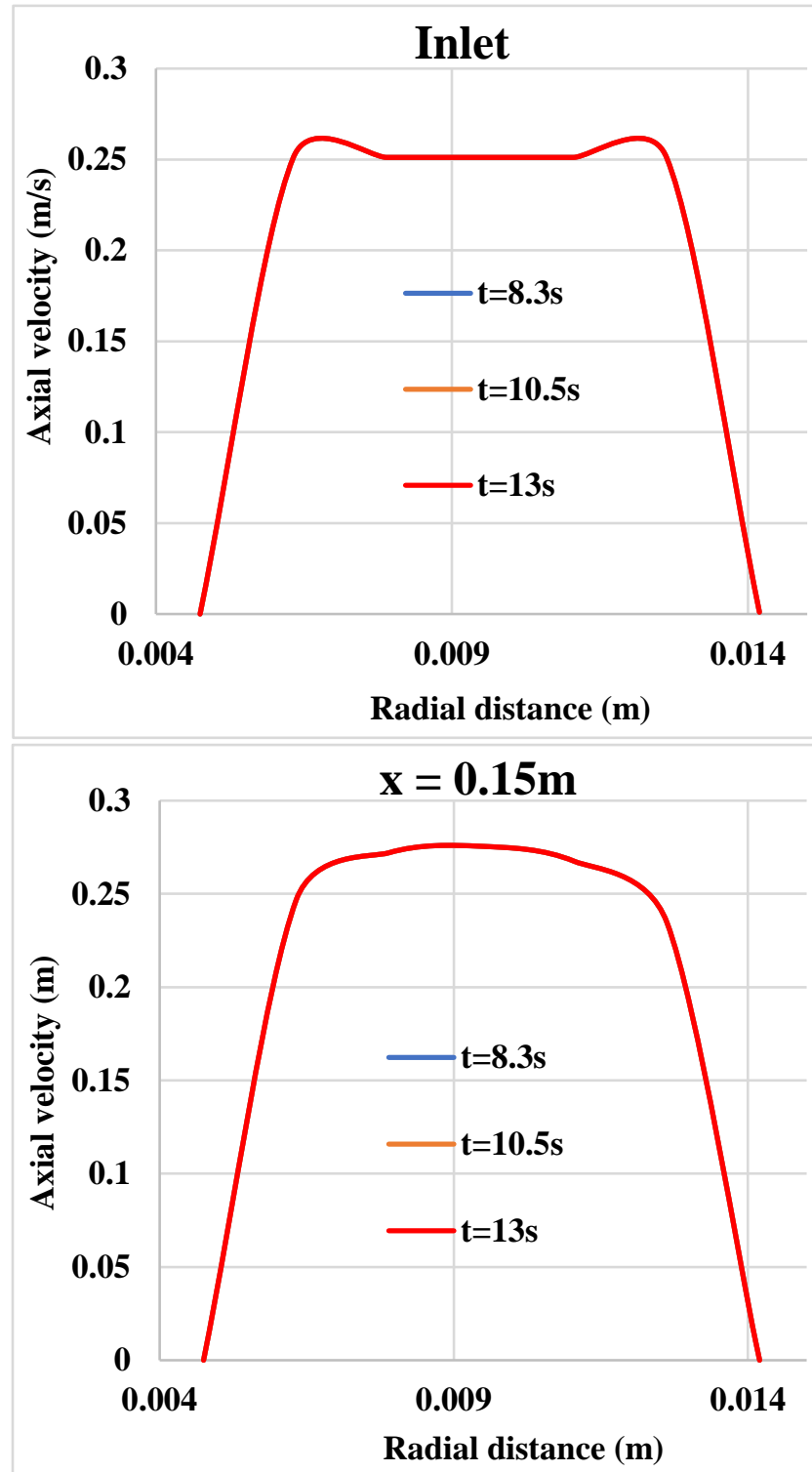
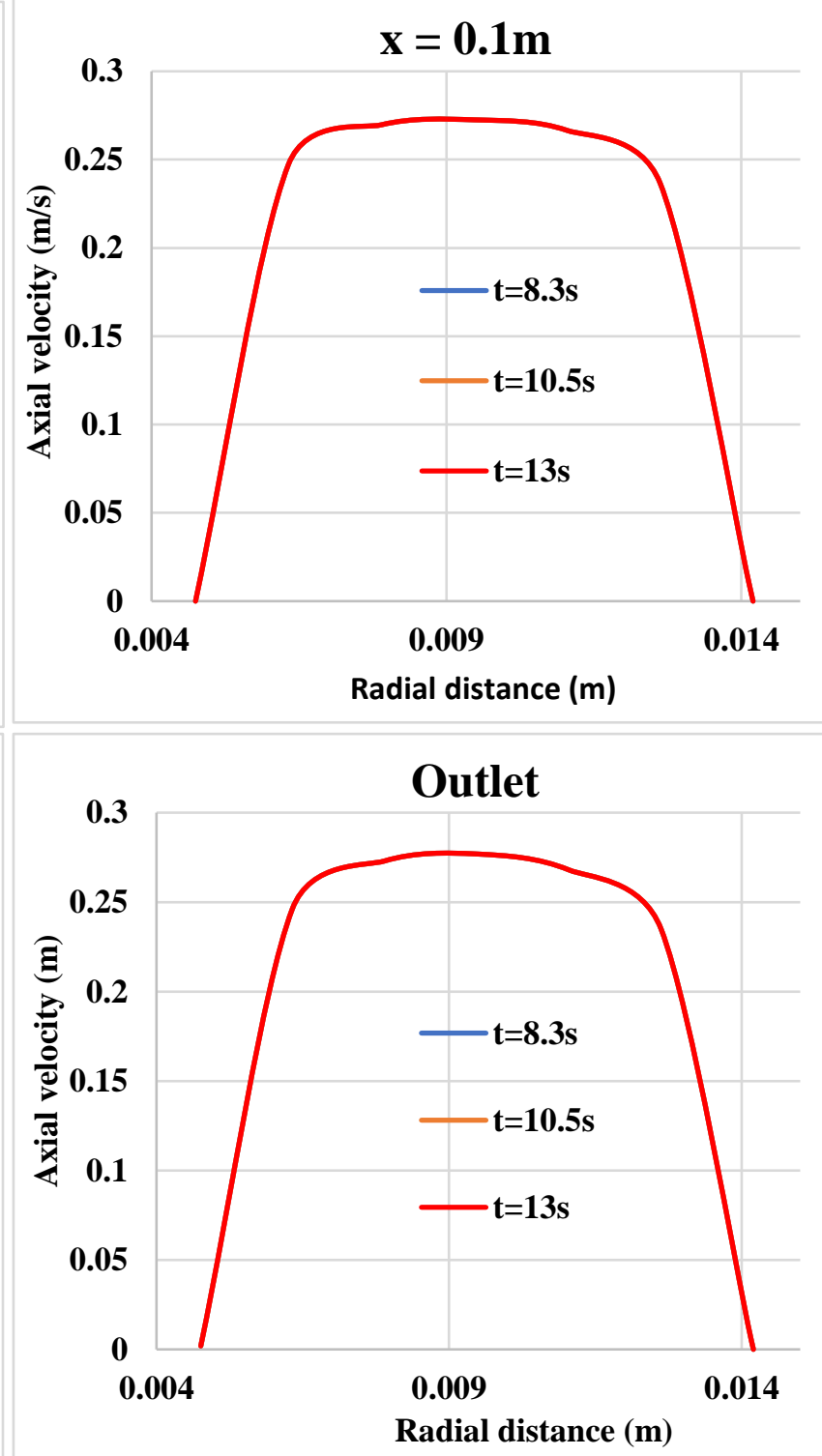

Figure 5.16: Axial velocity of the gas versus radial distance at various axial locations.

Despite the use of two-way coupling, instability/fluctuation in the flow field of the gas phase wasn't seen (Figs. 5.17 and 5.18). So, it was suspected that such phenomena appear in the flow as 
a result of turbulence dispersion and high particle loading. To validate this hypothesis more cases were simulated.

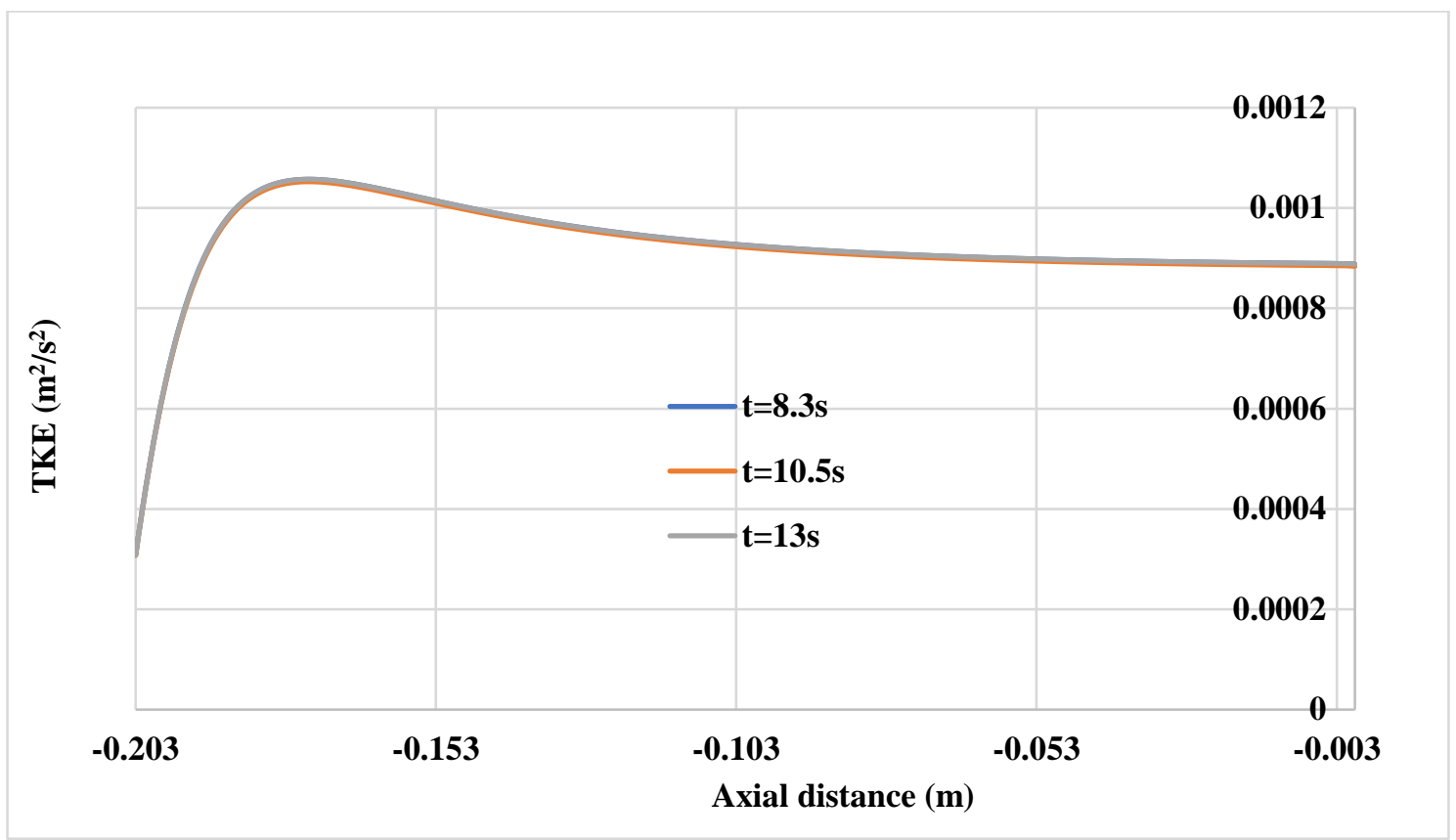

Figure 5.17: The TKE profile along with the boundary layer for Case 3.

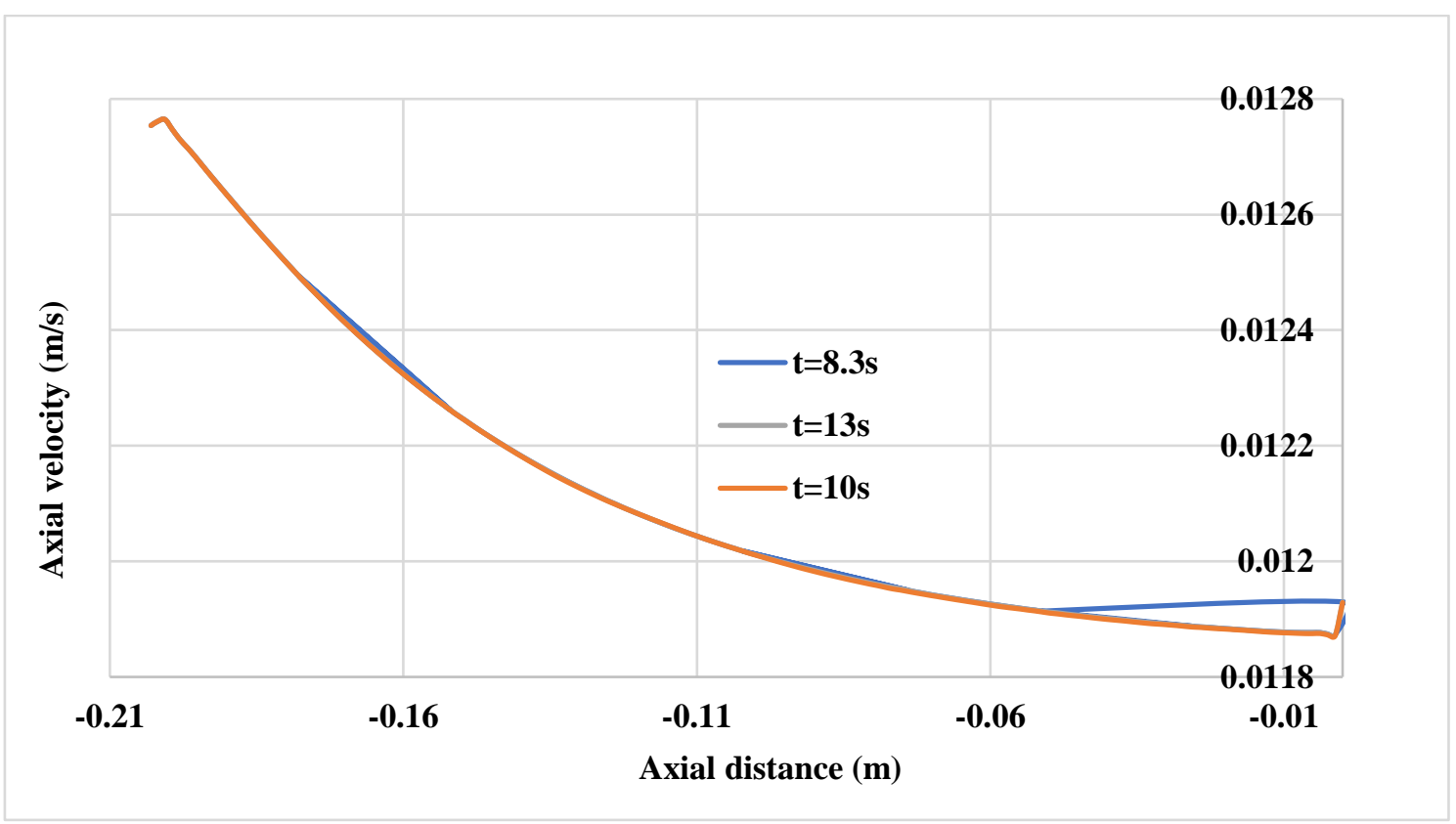

Figure 5.18: The axial gas velocity along with the boundary layer for Case 3. 


\subsubsection{Case 4: Low particle loading with turbulence dispersion and two-way coupling}

This case was modeled using two-way coupling with the incorporation of turbulence dispersion of the particles. Similar to the previous cases, the graphs for the axial velocity and the TKE have been plotted. The results differed from those obtained in the earlier cases. Specifically, the through observation of the DPM-contour in Fig. 5.19 showed the clustering of the particles in a different location near the wall.

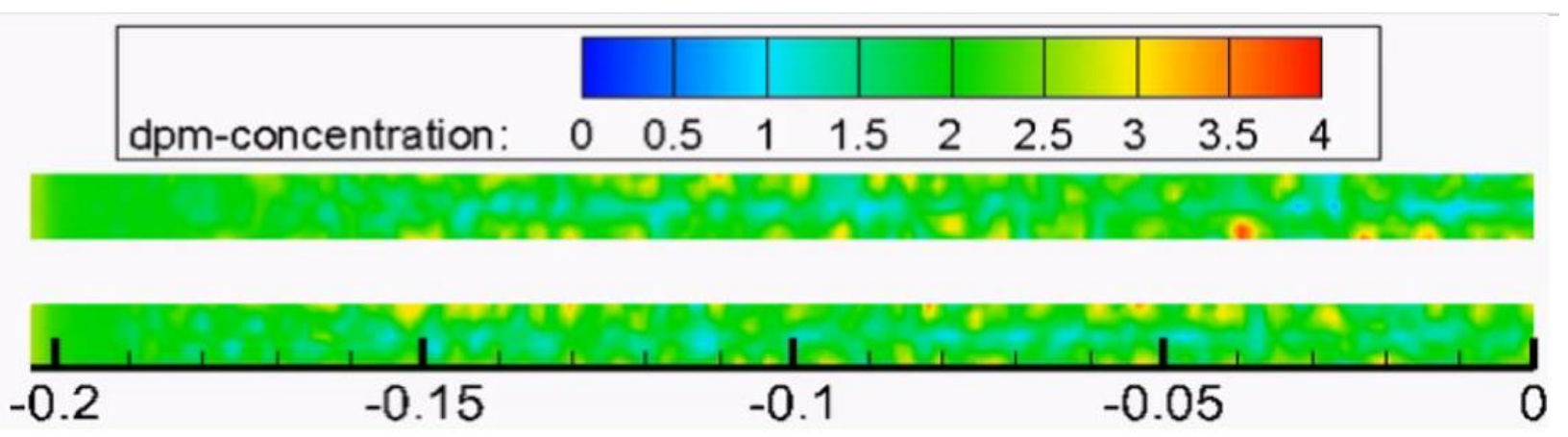

Figure 5.19: The DPM concentration contour for Case 4.

The axial velocity profiles of gas phase at various locations demonstrate the distortion (instability) in the flow field (Fig. 5.20). Also, the TKE and the axial velocity plots (Figs. 5.21 and 5.22) along the boundary layer show the flow field fluctuations.

Consequently, the results obtained from all three cases of low particle loading showed that the turbulence dispersion of the particles, along with the two-way coupling model, triggers the distortion of the flow field. The turbulence dispersion of the solid particles can impact the gas phase when two-phase interaction is turned on. 

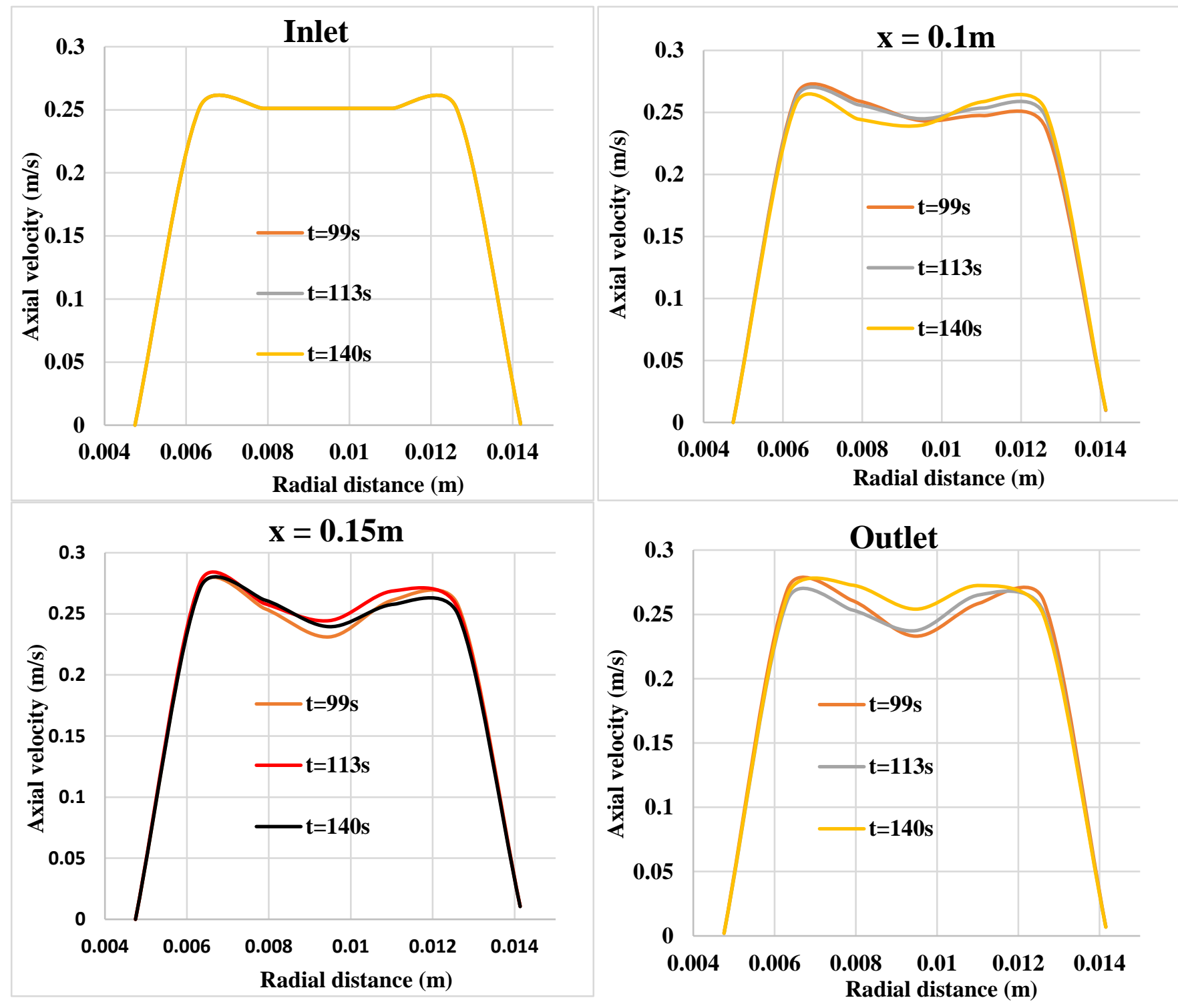

Figure 5.20: Axial velocity of the gas versus radial distance at various axial locations. 


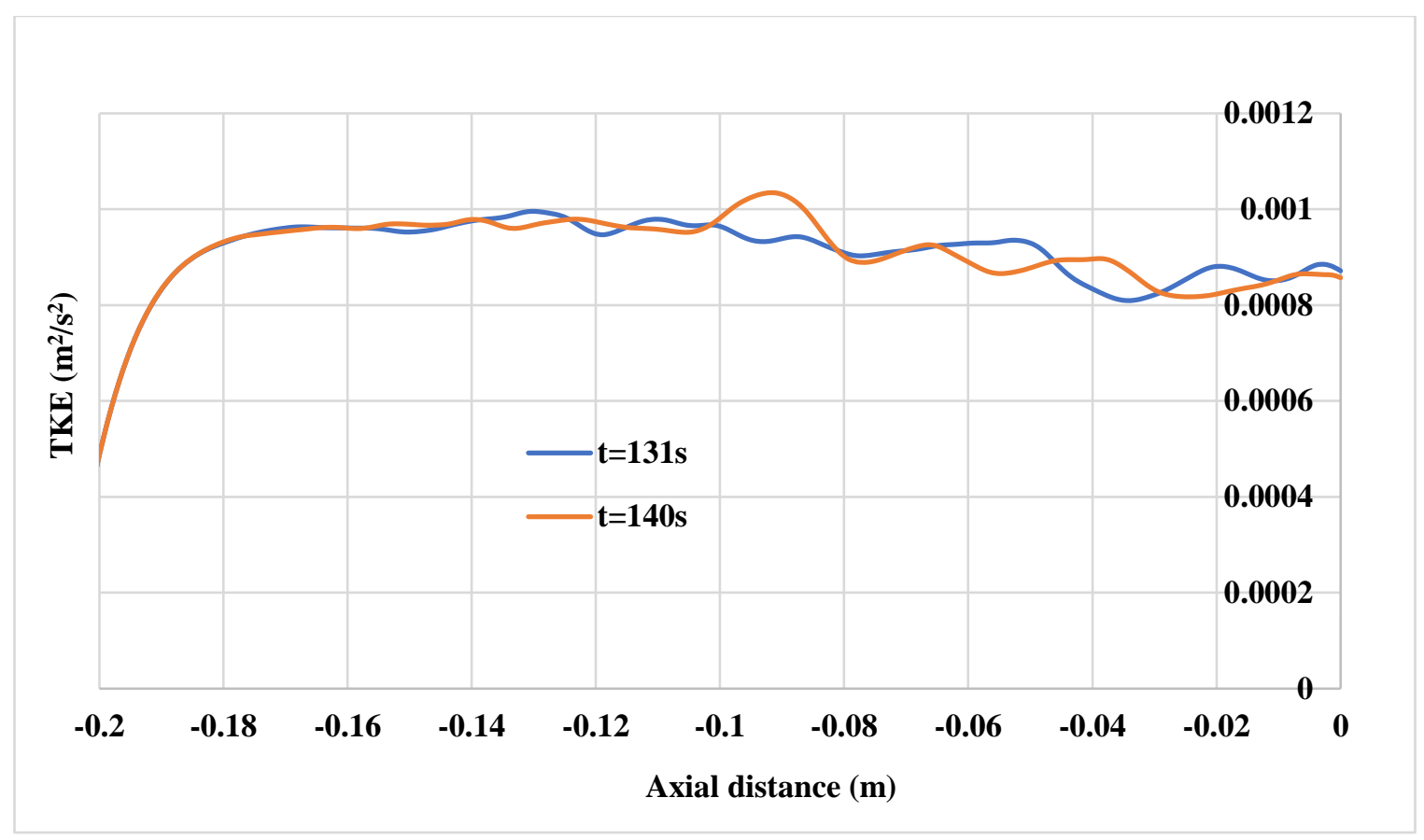

Figure 5.21: The TKE profile at the boundary layer for Case 4.

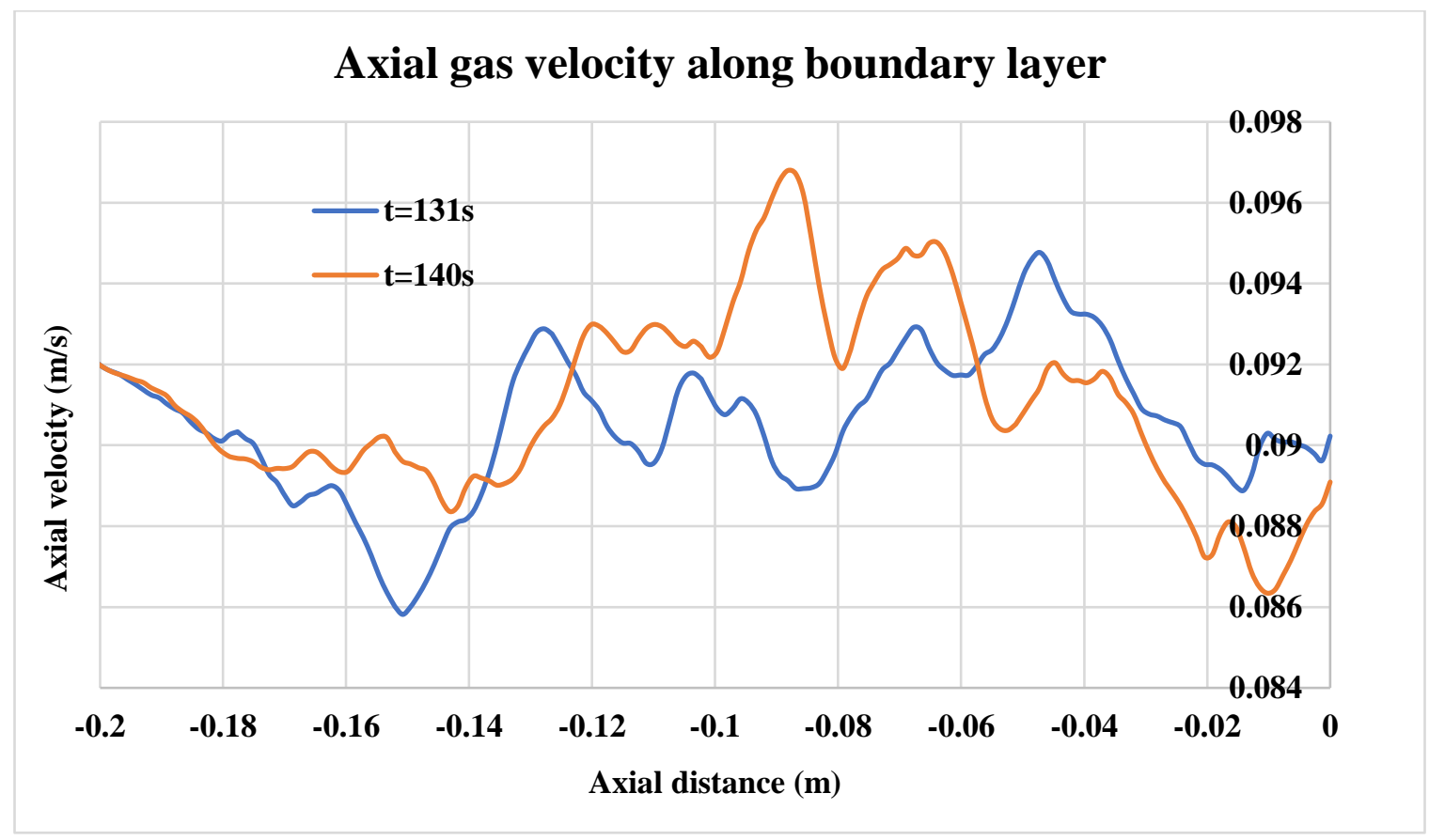

Figure 5.22: The axial velocity of the gas at the boundary layer for Case 4. 


\subsubsection{Case 5: High particle loading with turbulence dispersion and one-way coupling}

This case presents the results obtained for the high particle loading with the effect of turbulence dispersion of the particle but had no interaction between the phases. Because of no interaction between the phases, the effect of turbulence dispersion of particles was not found in the gas phase although the DRW model was turned on. The axial velocity profile, Fig. 5.24, and the TKE profile, Fig. 5.23, for this case resembled Case 2 .

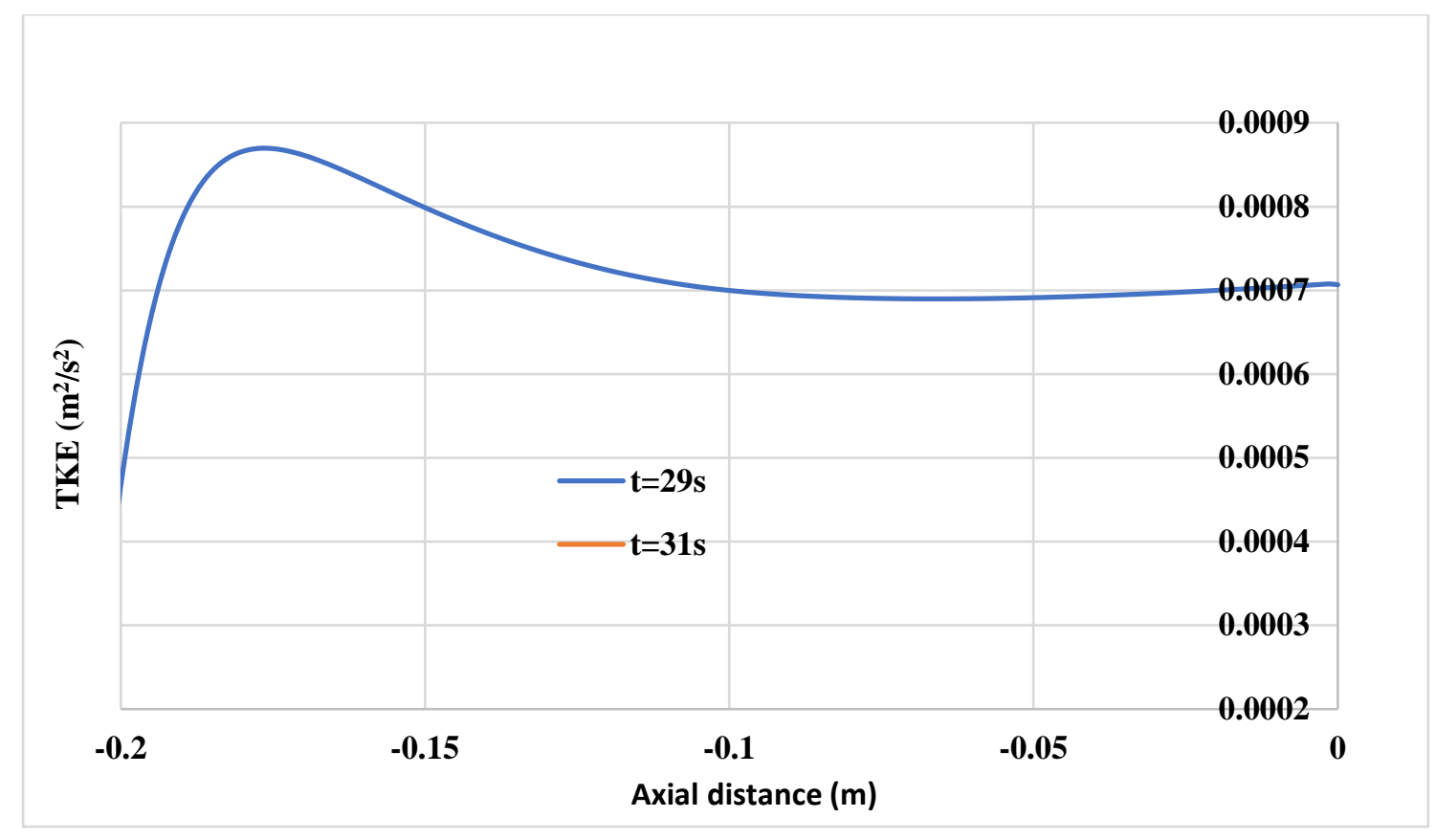

Figure 5.23: The TKE profile along with the boundary layer for Case 5 

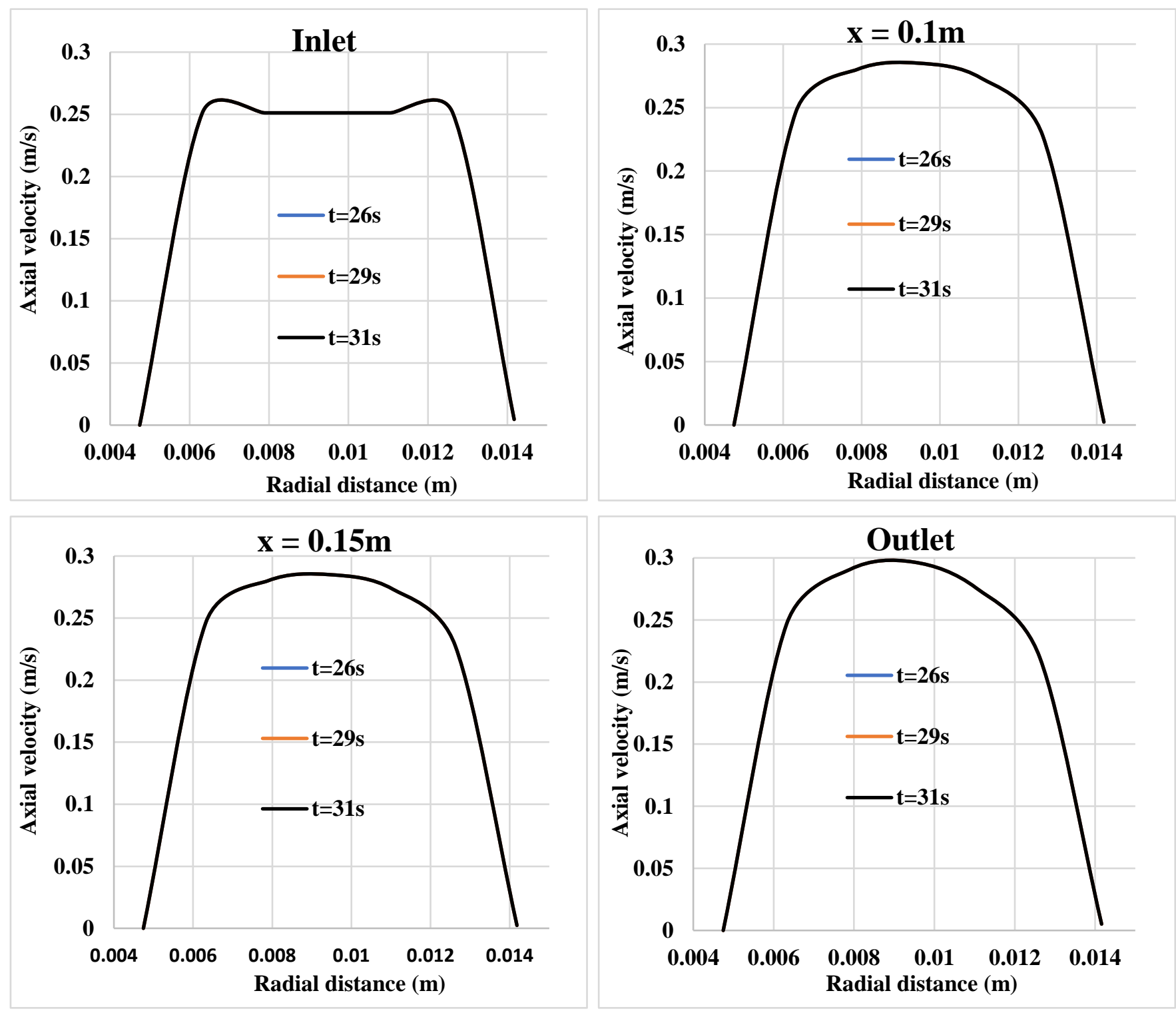

Figure 5.24: Axial velocity of the gas versus radial distance at various axial locations. 


\subsubsection{Case 6: High particle loading with no turbulence dispersion and two-way coupling}

Unlike Case 5, though Case 6 underlines the interaction between the phases, turbulence dispersion of the particle was not considered. All the parameters for this case are identical to Case 3 except for the particle mass flow rate. A distortion in the flow field was not expected since the turbulence dispersion was turned off. However, the random appearance and disappearance of the fluctuation in the flow field can be seen in the DPM concentration contour, Fig. 5.25. Also, the graphs in Fig. $5.26-5.28$ delineate the nature of the fluctuations. Such a unique observation is attributed to the particle volume fraction. Indeed, the mass flow rate for high particle loading is about ten times larger than that for the low particle loading. Hence, the clustering of the particle occurs, eventually causing the fluctuations/instability of the flow: the effect of particle clustering is transferred to the gas phase via the two-phase coupling.

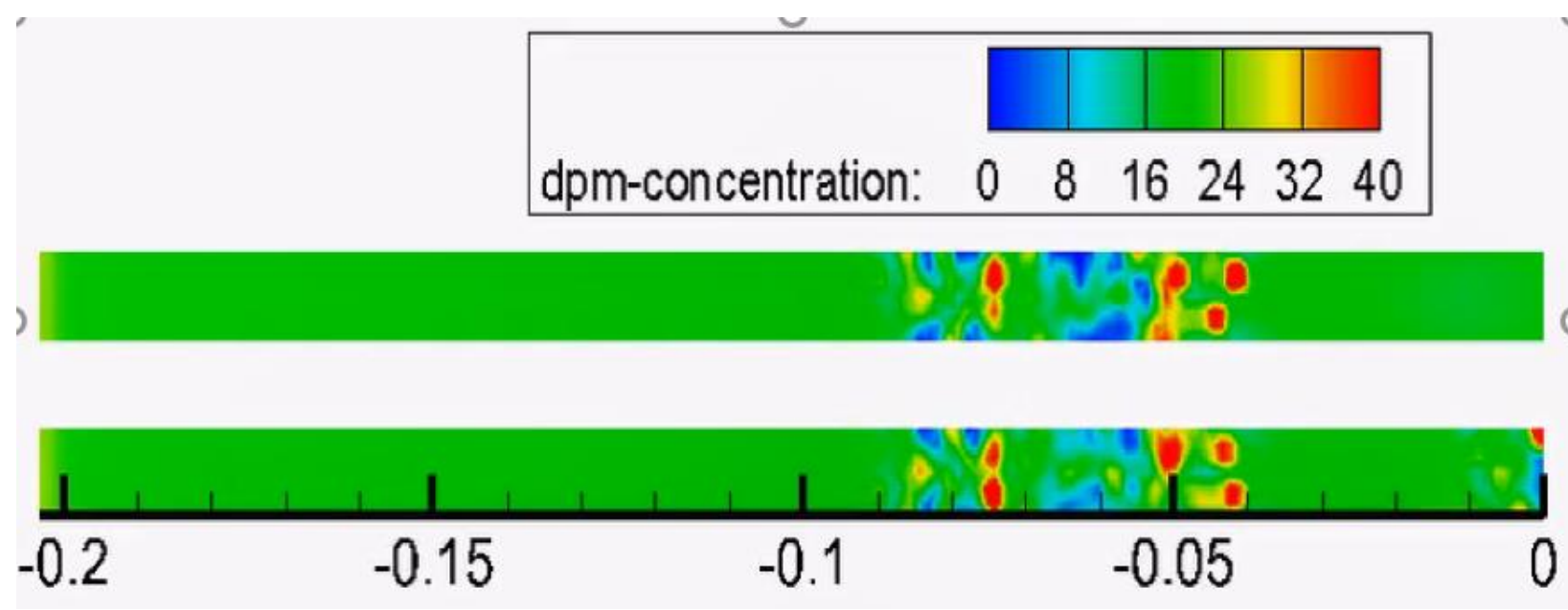

Figure 5.25: The DPM concentration contour for Case 6. 


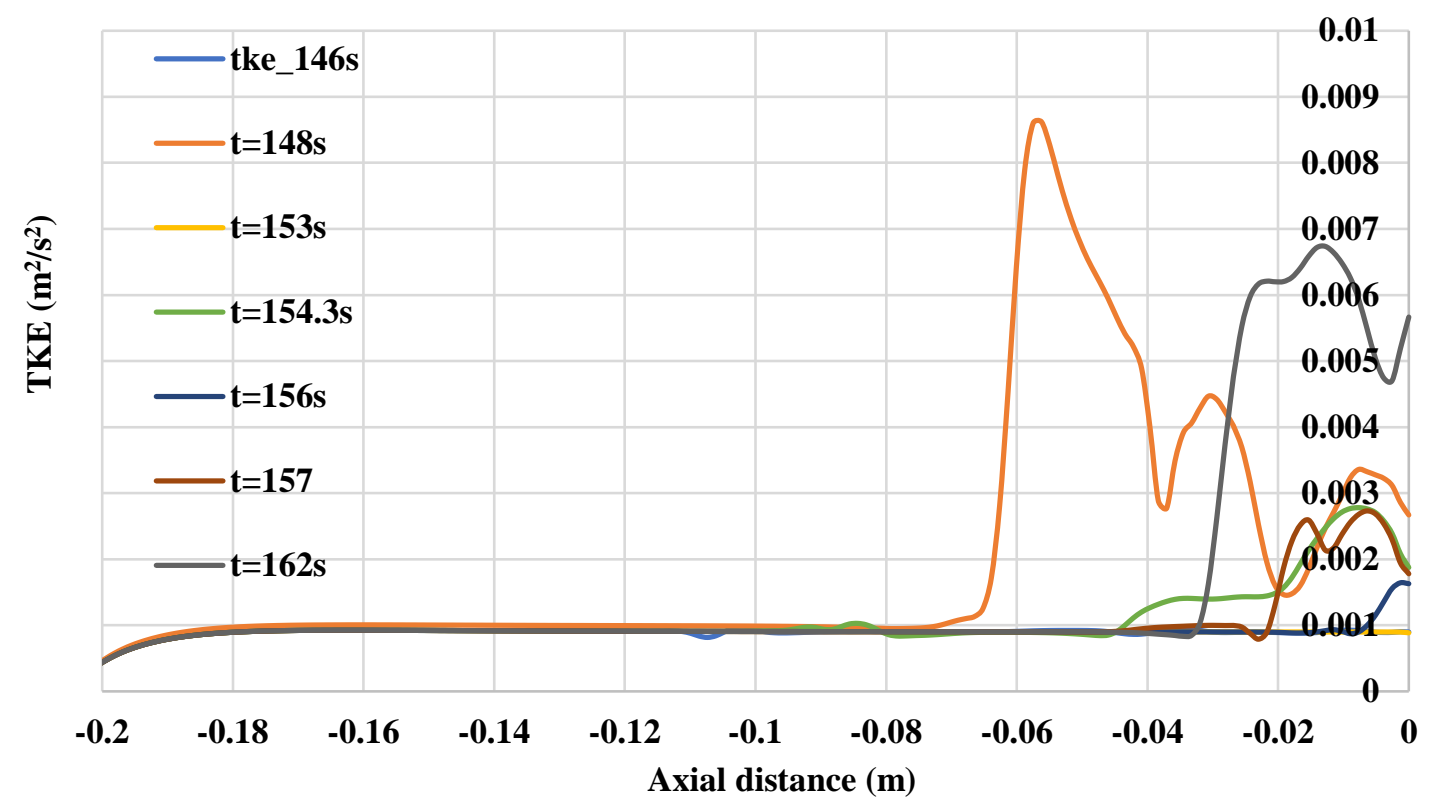

Figure 5.26: The TKE profile along with the boundary layer for Case 6.

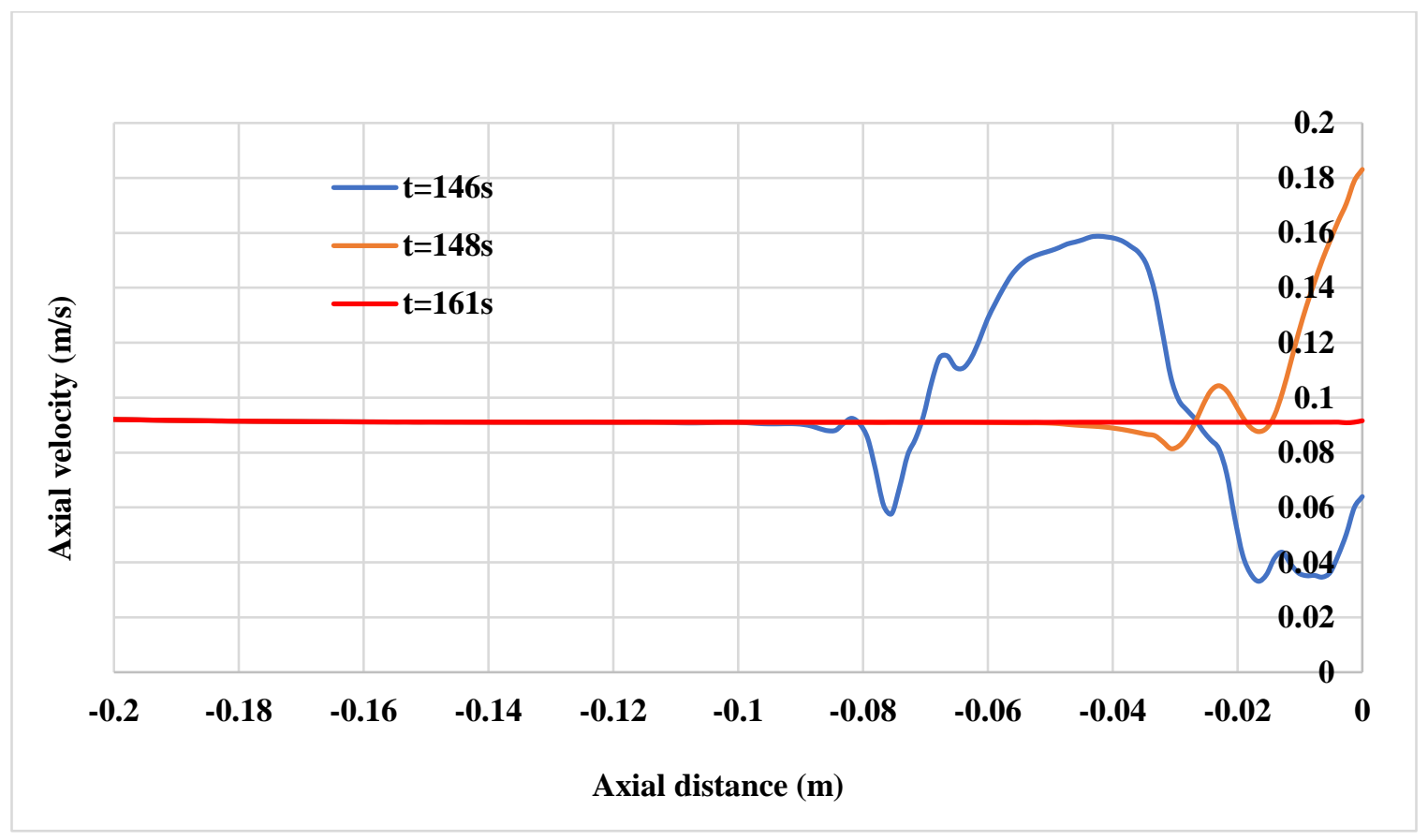

Figure 5.27: The axial velocity of gas along with the boundary layer for Case 6. 

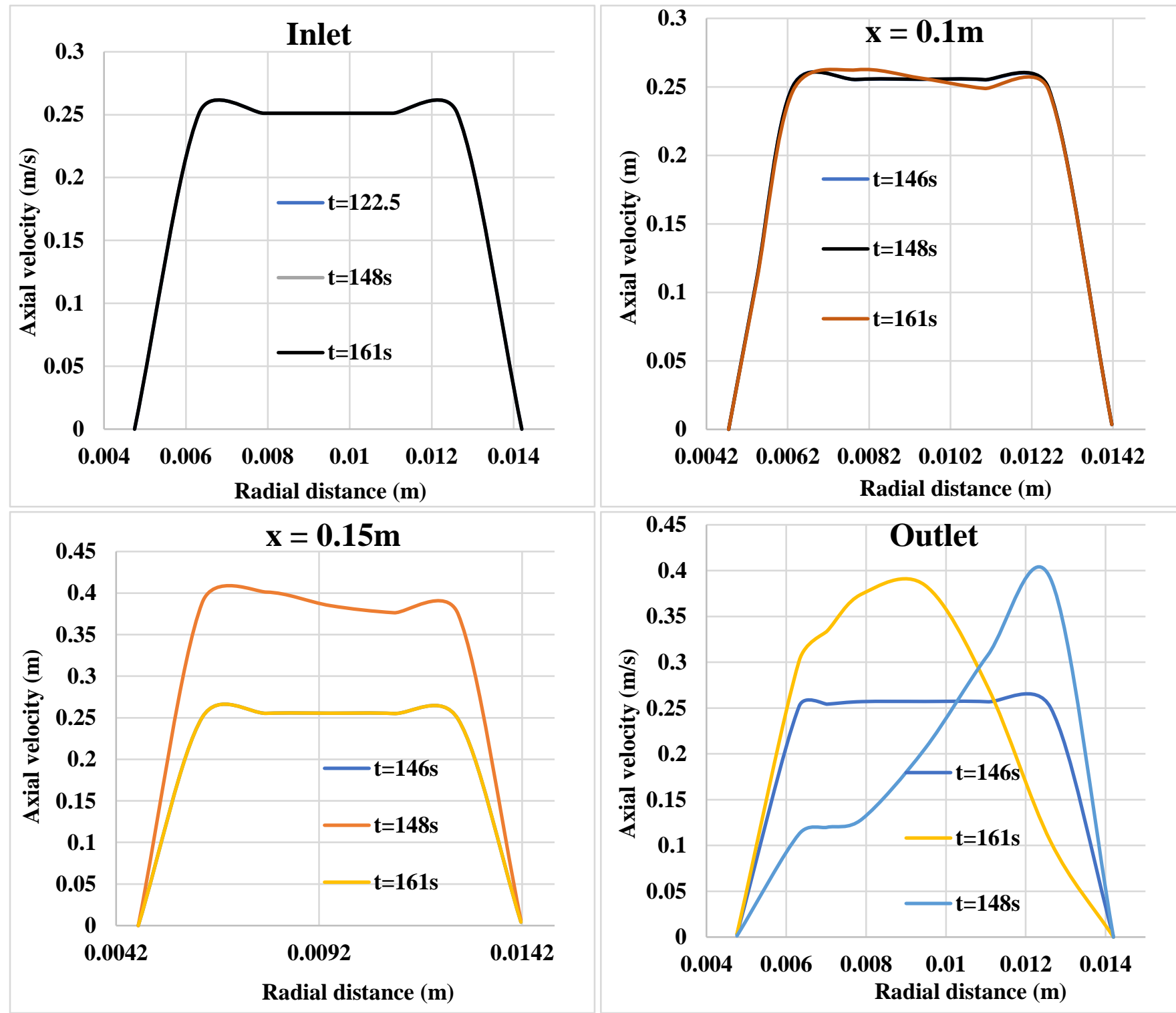

Figure 5.28: Axial velocity of the gas versus radial distance at various axial locations. 


\subsubsection{Case 7: High particle loading with turbulence dispersion and two-way coupling}

Finally, the turbulence dispersion of the particles is considered. The DPM concentration contour plot, Fig. 5.29, showed the gather/clustering of the particle at the various location near the wall. Also, the TKE and the axial velocity plot, Figs. 5.30 and 5.31, respectively, exhibited huge fluctuations along the boundary layer. Likewise, the velocity profile delineated in Fig. 5.32 shows the distortion of the flow field. The fluctuations of the velocity were not randomly repetitive, as in case 6 , but were sustained. Hence, activating turbulence dispersion of the particles enhanced and sustained the flow field distortion phenomenon.

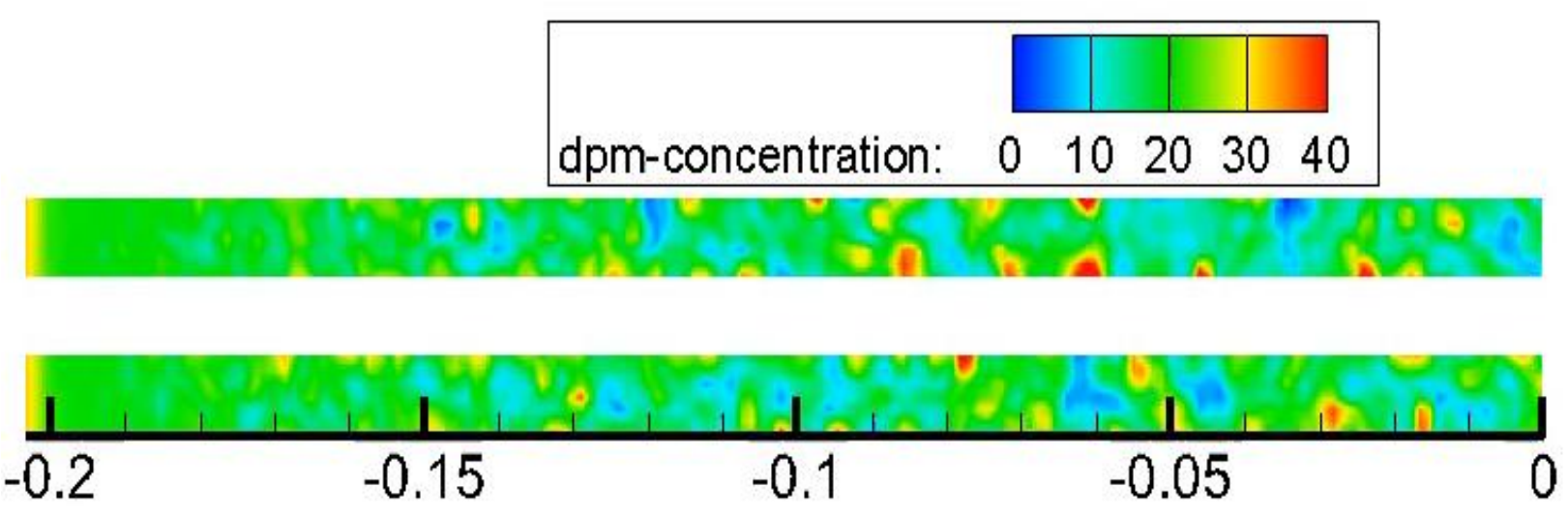

Figure 5.29: DPM concentration contour for Case 7. 


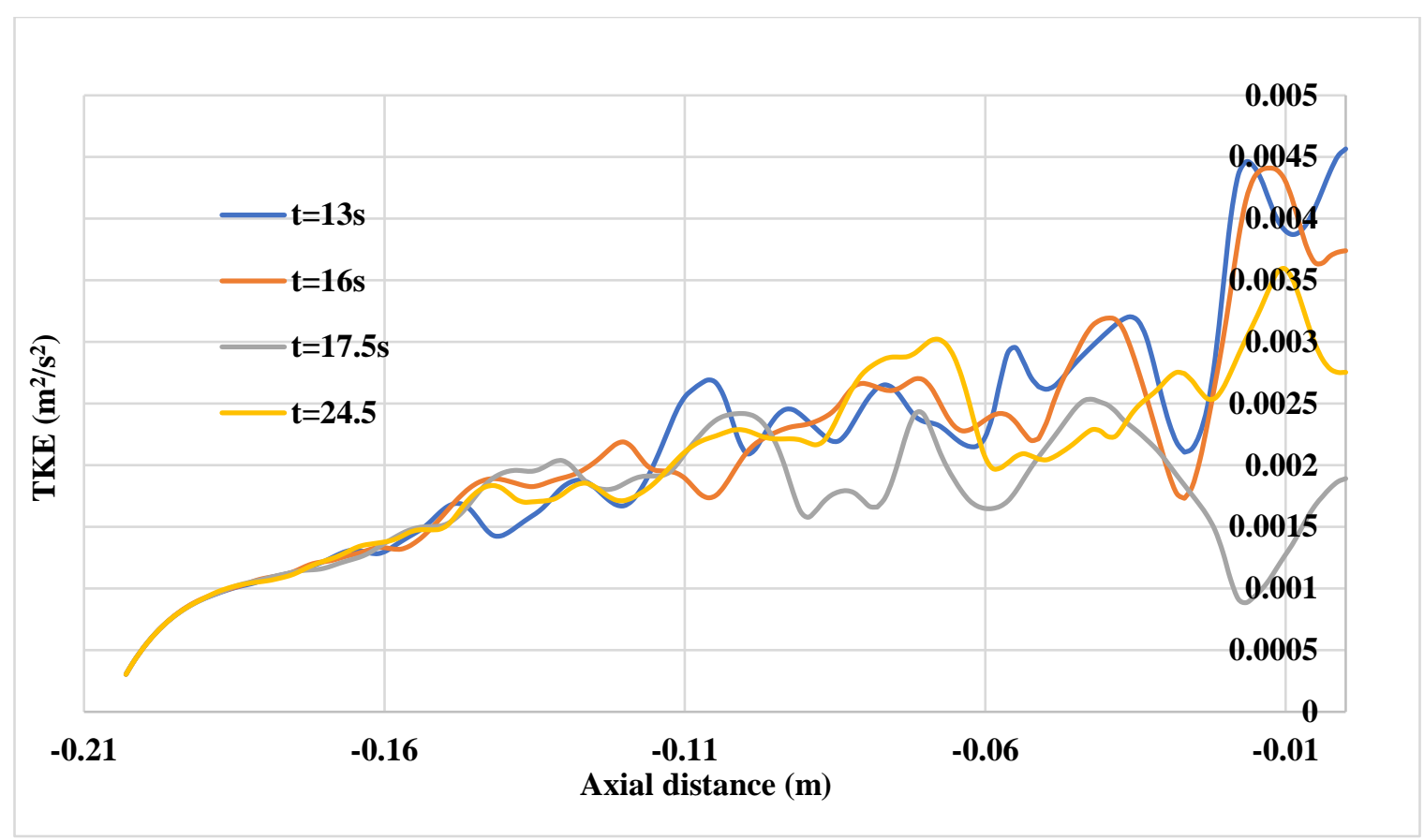

Figure 5.30: The TKE profile along the boundary line for Case 7.

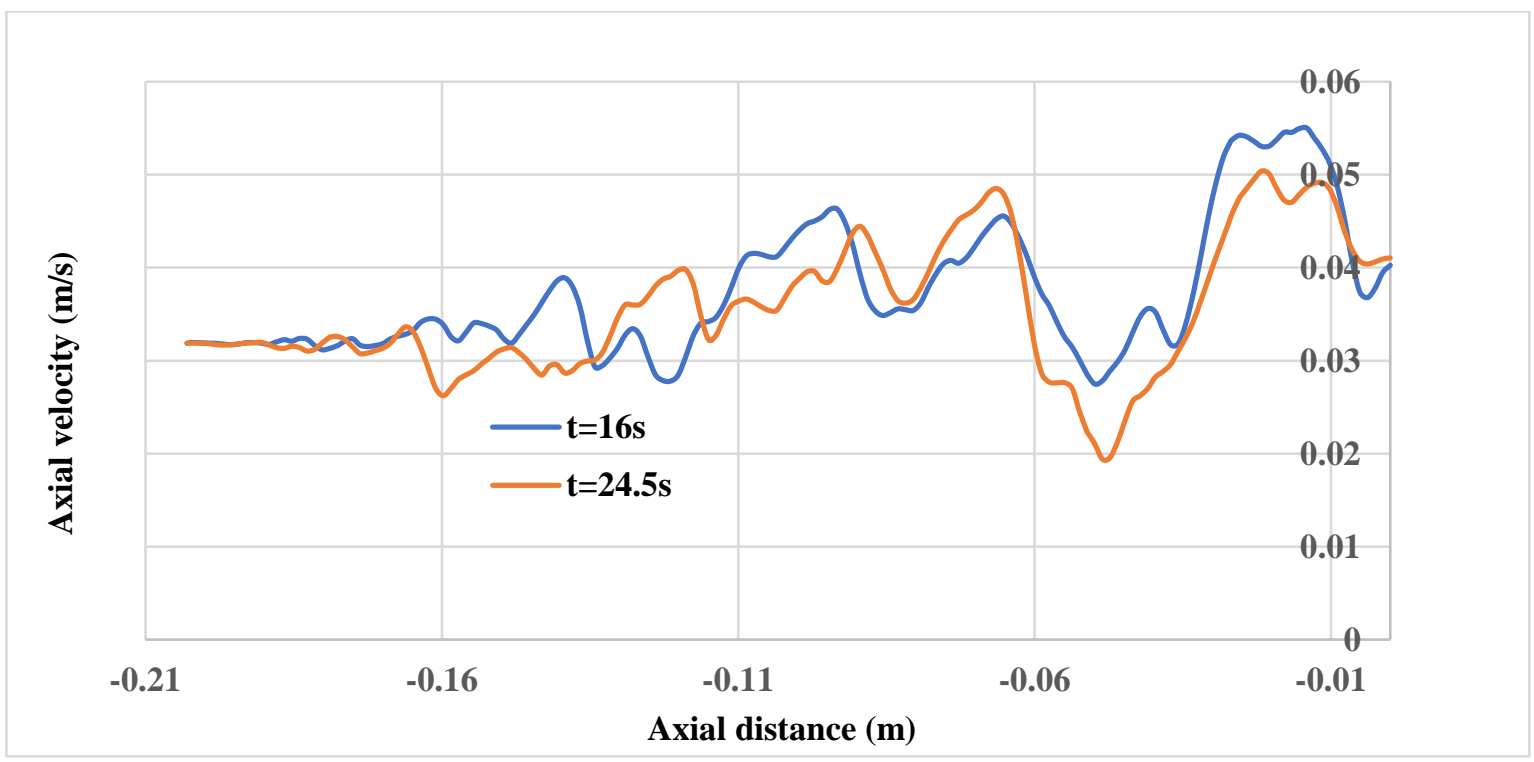

Figure 5.31: Velocity profile of the gas phase along the boundary line for Case 7 

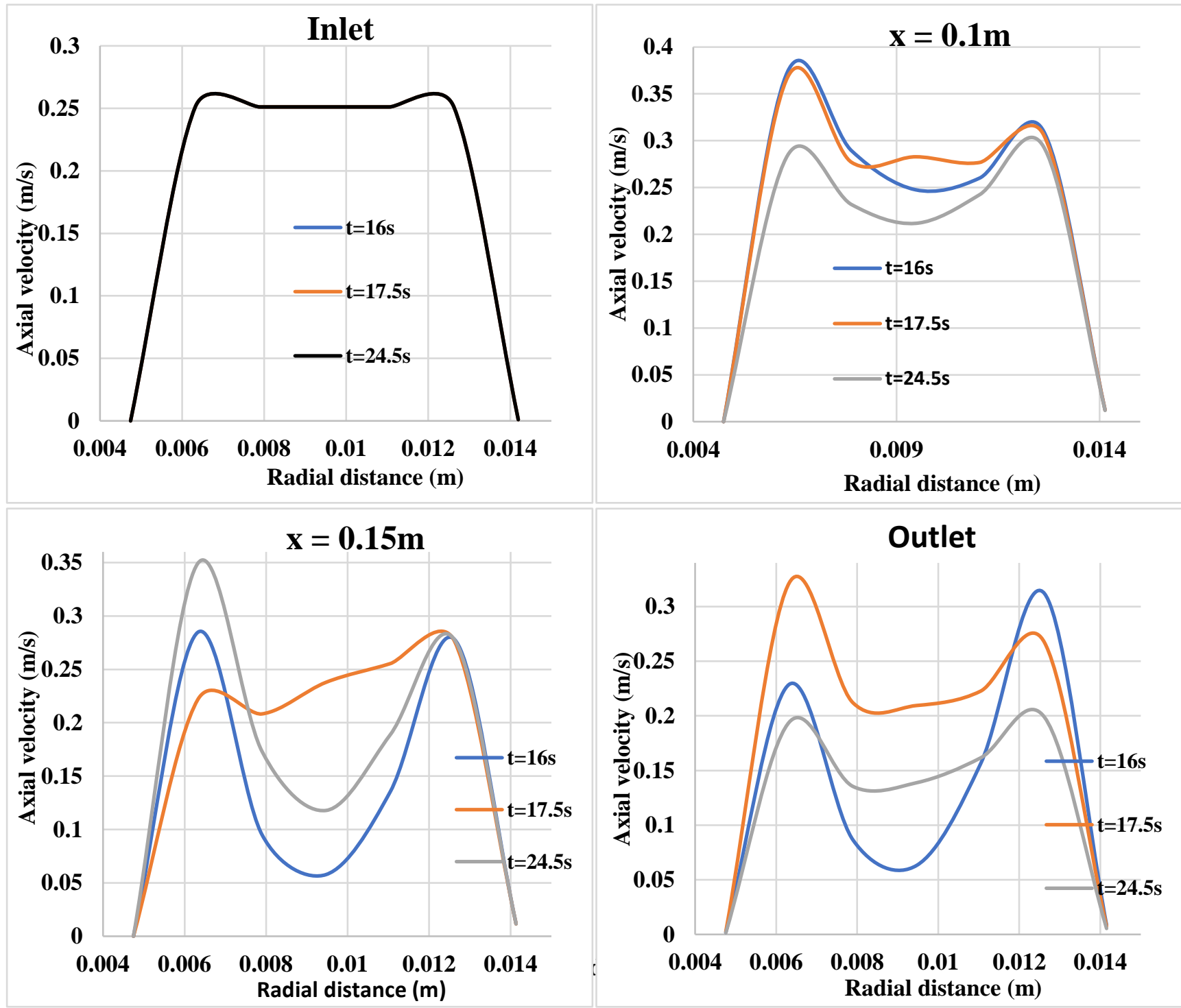


\section{Conclusion}

With an objective to understand the fundamental physics governing the gas-solid phase flow inside the uniform cross-section vertical annular pipe, seven different cases of gas-solid flow were developed. The role of phase coupling, particle loading, and turbulence dispersion was studied, along with the effect of gravity on the particle velocity. To match the SPOC experimental condition the operating pressure of the system of 15 bar was used.

Gravity was found to be causing the velocity lag between the phases. Despite having a moderate Reynolds number, the gas phase itself did not show any turbulence in the flow. For the cases with high (5) and low (2) particle loading, one-way coupling, and turbulence dispersion the presence of particles on the flow field did not show any influence on the gas-phase. It was therefore theorized that one-way coupling between the phases does not account for any effects caused by the solid phase on the gas flow field despite having different particle loading.

In addition, in the flow with two-way interaction but no turbulence dispersion, different results have been discovered. Specifically, for the low particle loading, Case 3, the flow field seemed to have no turbulence, while Case 6 with high particle loading showed random repetition in flow field fluctuation/distortion. High particle loading was found to cause the particle clustering in the lateral case and was attribute to the difference.

Finally, for a two-way coupling with turbulence dispersion, Cases 4 and 7, sustained fluctuations of the flow were observed for both cases. Hence, it is concluded that the phase-coupling, turbulence dispersion, and particle loading provides a significant role in changing the flow. The fluctuations of the flow field are caused by high particle loading, which is further enhanced by the presence of the particle turbulence dispersion. 


\section{Recommendation}

Based on the numerical simulation this thesis presents the effect of particle loading, turbulence dispersion of the particle, and phase coupling on the flow field of gas-phase. Results obtained are unique and provides the fundamental explanation for the many questions which were left unanswered before: regarding two-phase gas-solid flow. However, there are still some questions that seek an answer. First, this is a numerical study hence, to increase the validity of the research experiments can be conducted and results thus generated should be compared to those obtained from this study.

Also, the simulations are conducted by injecting the particles with a uniform size distribution of $65 \mu \mathrm{m}$. The influence of various sizes on the flow filed is not studied. Therefore, further study can be conducted by using a particle size greater and smaller than $65 \mu \mathrm{m}$.

Besides, this study is based on a fixed Reynold's number. The operating pressure is 15 bar. This study can be continued to understand the influence of pressure and hence Reynolds's number on the flow.

Lastly, this thesis presents the cases with a different mass flow rate of the particle. For a low particle loading, the volume fraction of the particle is $0.13 \%$ while for high particle loading it is $1.2 \%$. Based on the study by Elghobashi [42] it is recommended to use two-way coupling for a flow with the particle volume fraction of about $0.1 \%$. However, for a higher volume fraction fourway coupling is suggested to use. Although the effect of one-way and two-way interaction is considered in this research, particle-particle interaction is neglected. Since the clustering of the particle was observed, studies based on particle-particle interaction can be conducted. Besides, even for the low particle loading case, the distortion in the flow field is still observed while using the two-way coupling and turbulence dispersion. One can dig even more to understand this flow phenomenon. 


\section{References}

[1] C.E. Brennen, "Introduction to multiphase flow," pp. 19-22 in "Fundamentals of multiphase flows," London, Cambridge University Press, 2005.

[2] S. Balachandar, J.K. Eaton, "Turbulent dispersed multiphase flow," Annual Review of Fluid Mechanics, vol. 42, pp. 111-133, 2010.

[3] J.R. Fanchi, J.P. Seidle, "Multiphase flow in porous media," pp. 685-728 in "Multiphase flow handbook," CRC Press, Boca Raton, 2016.

[4] A. Faghri, Y. Zhang, "Two-phase flow and heat transfer," pp. 853-949 in Transport phenomena in multiphase systems, Academic Press, London, UK, 2006.

[5] S.R. Turns, An introduction to combustion: concepts and applications, pp. 8-9, McGrawHill, New York, USA, 2011.

[6] TR. Anderson, E. Hawkins, P.D. Jones, "CO2, the greenhouse effect and global warming: from the pioneering work of Arrhenius and Callendar to today's Earth System Models," Endeavour, vol. 40, no. 3, pp. 1-10, 2016.

[7] BP, "Primary energy: consumption by fuel," BP Statistical Review of World Energy, vol. 68, pp. 8-12, 2019.

[8] BP, "Coal," BP Statistical Review of World Energy, vol.68, pp. 42-47, 2019.

[9] P. Mathieu, "Oxyfuel combustion systems and technology for carbon dioxide $\left(\mathrm{CO}_{2}\right)$ capture in power plants," Woodhead Publishing Series in Energy, vol. 1, pp. 283-319, 2010.

[10] R. Stanger, T. Wall, R. Spörl, M. Paneru, S. Grathwohl, M. Weidmann, G. Scheffknecht, D. McDonald, K. Myöhänenx, J. Ritvanen, S. Rahiala, T. Hyppänen, J. Mletzko, A. Kather, S. 
Santos, "Oxyfuel combustion for $\mathrm{CO}_{2}$ capture in power plants," International Journal of Greenhouse Gas Control, vol. 40, pp. 55-125, 2015.

[11] S.M. Carpenter, H.A. Long III, "Integration of carbon capture in IGCC systems," Integrated Gasification Combined Cycle Technologies, vol. 13, pp. 445-463, 2017.

[12] A. Gopan, B.M. Kumfer, R.L. Axelbaum, "Effect of operating pressure and fuel moisture on net plant efficiency of a staged, pressurized oxy-combustion power plant," International Journal of Greenhouse Gas Control, vol. 39, pp. 390-396, 2015.

[13] H. Hagi, M. Nemer, Y.L. Moullec, C. Bouallou, "Towards second generation oxy-pulverized coal power plants: Energy penalty reduction potential of pressurized oxy-combustion systems," Energy Procedia, vol. 63, pp. 431-439, 2014.

[14] M.D. Giacinto, F. Sabetta, R. Piva, "Two-way coupling effects in dilute gas-particle flows," Journal of Fluid Engineering, vol. 104, pp. 304-311, 1982.

[15] X. Liu, B.J. Glasser, "A parametric investigation of gas-particle flow in a vertical duct," American Institute of Chemical Engineers Journal, vol. 53, pp. 940-956, 2005.

[16] J.L. Sinclair, R. Jackson, "Gas-particle flow in a vertical pipe with particle-particle interaction," American Institute of Chemical Engineers Journal, vol. 35, pp. 1473-1486, 1989.

[17] A.I. Kartushinsky, E.E. Michaelides, Y.A. Rudi, S.V. Tisler, I.N. Shcheglov, "Numerical simulation of three-dimensional gas-solid particle flow in a horizontal pipe," American Institue of Chemical Engineers Journal, vol. 57, pp. 2977-2988, 2011.

[18] M. Rhodes, S. Zhou, H. Benkreira, "Flow of dilute gas-particle suspensions," American Institue of Chemical Engineers Journal, vol. 38, pp. 1913-1915, 1992.

[19] Y. Ding, Z. Wang, M. Ghadiri, D. Wen, "Vertical upward flow of gas-solid two-phase mixtures through monolith channels," Powder Technology, vol. 153, pp. 51-58, 2005. 
[20] F. Xia, Z. Yang, A. Adeosun, A. Gopan, B.M. Kumfer, R.L. Axelbaum, "Pressurized oxycombustion with low flue gas recycle: Computational fluid dynamic simulations of radiant boilers," Fuel, vol. 181, pp. 1170-1178, 2016.

[21] J. Hong, R. Field, M. Gazzino, A.F. Ghoniem, "Operating pressure dependence of the pressurized oxy-fuel combustion power cycle," Energy, vol. 35, pp. 5391-5399, 2010.

[22] J.A. Lasek, K. Głód, M. Janusz, K. Kazalski, J. Zuwała, "Pressurized oxy-fuel combustion: a study of selected parameters," Energy and Fuels, vol. 26, pp. 6492-6500, 2012.

[23] G. Udochukwu, "Computational design of staged pressurized oxy-coal," Master's Thesis in Aerospace Engineering, West Virginia University, Morgantown, WV, USA, 2019.

[24] A. Boateng, "Combustion and flame," pp. 107-143 in "Rotaty kilns," New York, Butterworth-Heinemann, 2016.

[25] R.L. Axelbaum, B.M. Kumfer, A. Gopan, Z. Yang, J. Phillips, B. Pint, "Staged, highpressure oxy-combustion technology: development and scale-up," U.S Department of Energy, Dec. 29 2017, doi:10.2172/1415166 .

[26] B.E. Rapp, "Computational fluid dynamics," pp. 609-622 in "Microfluidics: modelling, mechanics and mathematics," London, Matthew Deans, 2017.

[27] B. Andersson, R.L. Andersson, L. Hakansson, M. Mortensen, R. Sudiyo, B.V. Wachem, "CFD simulations," pp. 1-5 in "Computational fluid dynamics for engineers," New York, Cambridge University Press, 2012.

[28] H.H. Hu, "Computational fluid dynamics," pp. 421-473 in "Fluid mechanics," London, Academic press, 2012.

[29] S. Thabet, T.H. Thabit, "Computational fluid dynamics: science of the future," International Journal of Research and Engineering, vol. 5, pp. 430-433, 2018. 
[30] I. Sadrehaghighi, "Turbulence modeling - a review," pp. 1-19 in CFD open series, United states, 2020.

[31] J. Mathieu, J. Scott, "An introduction to turbulence," pp. 1-39 in "An introduction to turbulent flow," New York, Cambridge University Press, 2000.

[32] W. Rodi, "DNS and LES of some engineering flows," Fluid Dynamics Research, vol. 38, pp. 145-173, 2006.

[33] G. Alfonsi, "On direct numerical simulation of turbulent flows," Applied Mechanics Reviews, vol. 64, pp. 1-33, 2011.

[34] H.K. Versteeg, W. Malalasekera, "Direct numerical simulation," pp. 111-113 in "An introduction to computational fluid dynamics," London, Pearson Education Limited, 2007.

[35] H.K. Versteeg, W. Malalasekera, "Large eddy simulation," pp. 98-109 in "An introduction to computational fluid dynamics," London, Pearson Education Limited, 2007.

[36] ANSYS Inc., "Large Eddy Simulation (LES) model," pp. 99-108 in "ANSYS Fluent theory guide," Canonsburg, 2013.

[37] J. Smagorinsky, "General circulation experiments with the primitive equations," Monthly Weather Review, vol. 91, pp. 99-164, 1963.

[38] J. Frohlich, D.V. Terzi, "Hybrid LES/RANS methods for the simulation of turbulent flows," Progress in Aerospace Sciences, vol. 44, pp. 349-377, 2008.

[39] F.R. Menter, "Two-equation eddy-viscosity turbulence models for engineering applications," AIAA Journal, vol. 32, pp. 1598-1605, 1994.

[40] J. Blazek, "Turbulence modeling," pp. 213-244 in "Computational fluid dynamics: principles and applications," New York, Butterworth-Heinemann, 2015. 
[41] Z. Yinghui, L. Xingying, G. Jinsen, "Modeling of gas-solid flow in a CFB riser based on computational particle fluid dynamics," Petroleum Science, vol. 9, pp. 535-543, 2012.

[42] S. Elghobashi, "An updated classification map of particle-laden turbulent flows," IUTAM symposium on computational multiphase flows, Argonne National laboratory, Lemont, IL, USA, Oct. 4-7, 2004.

[43] H. Lu, X. Guo, W. Zhao, X. Gong, J. Lu, "Experimental and CPFD numerical study on hopper discharge," Industrial and Engineering Research, vol. 53, pp. 12160-12169, 2014.

[44] H. Ariyaratne, E. Manjula, C. Ratnayake, C. Morten, "CFD approaches for modeling gassolids multiphase flows," Linköping electronic conference, Finland, Sept. 12-16, 2016.

[45] G.F. Oweis, S.L. Ceccio, Y. Matsumoto, C. Tropea, I.V. Roisman, Y. Tsuji, R. Luczkowski, T.R. Troutt, J. K. Eaton, F. Mashayek, "Multiphase interactions," pp. 12.1-12.134 in "Multiphase flow handbook, New York, USA, CRC Press, 2006.

[46] ANSYS Inc.,"Discrete phase," pp. 373-463 in ANSYS Fluent theory guide, Canonsburg, PA, USA, 2013.

[47] I. Komasawa, R. Kuboi, T. Otake, "Measurement of turbulence of liquid by continual pursuit of tracer particle motion," Chemical Engineering Science, vol. 29, pp. 641-659, 1974.

[48] D. S. Jang, S. Acharya, "Calculation of particle dispersion due to turbulence in elliptical flows," American Institue of Chemical Engineers Journal, vol. 34, no. 3, pp. 514-518, 1988.

[49] B.M. Kumfer, R.L. Axelbaum, A. Gopan, "Effect of operating pressure and fuel moisture on net plant efficiency of a staged, pressuried oxy-combustion power plant," International Journal of Greenhouse Gas Control, vol. 39, pp. 390-396, 2015. 